Mathematische Zeitschrift 234 (2000), 9-52 — DOI: 10.1007/s002090050502

\title{
DUAL AFFINE QUANTUM GROUPS
}

\author{
FABIO GaVARINI \\ Università degli Studi di Roma "Tor Vergata" — Dipartimento di Matematica \\ Via della Ricerca Scientifica 1, I-00133 Roma - ITALY
}

\begin{abstract}
Let $\hat{\mathfrak{g}}$ be an untwisted affine Kac-Moody algebra, with its Sklyanin-Drinfel'd structure of Lie bialgebra, and let $\hat{\mathfrak{h}}$ be the dual Lie bialgebra. By dualizing the quantum double construction - via formal Hopf algebras - we construct a new quantum group $U_{q}(\hat{\mathfrak{h}})$, dual of $U_{q}(\hat{\mathfrak{g}})$. Studying its specializations at roots of 1 (in particular, its classical limits), we prove that it yields quantizations of $\hat{\mathfrak{h}}$ and $\widehat{G}^{\infty}$ (the formal group attached to $\hat{\mathfrak{g}})$, and we construct new quantum Frobenius morphisms. The whole picture extends to the untwisted affine case the results known for quantum groups of finite type.
\end{abstract}

\section{Introduction}

\section{"Dualitas dualitatum et omnia dualitas" \\ N. Barbecue, "Scholia"}

Let $\hat{\mathfrak{g}}$ be an untwisted affine complex Kac-Moody algebra, with the Sklyanin-Drinfel'd structure of Lie bialgebra; let $\hat{\mathfrak{h}}$ be its dual Lie bialgebra. Let $R$ be the subring of complex rational functions having no poles at roots of 1 . Let $U_{q}(\hat{\mathfrak{g}})$ be the quantum group - over the field $\mathbb{C}(q)$ - associated to $\hat{\mathfrak{g}}$ : then there exists an integer form $\mathfrak{U}(\hat{\mathfrak{g}})$ of $U_{q}(\hat{\mathfrak{g}})$ over $R$ which for $q \rightarrow 1$ specializes to $U(\hat{\mathfrak{g}})$ as a Poisson Hopf coalgebra (cf. [Lu2]). On the other hand, another integer form $\mathcal{U}(\hat{\mathfrak{g}})$ exists which for $q \rightarrow 1$ specializes (as a Poisson Hopf algebra) to $F[\widehat{H}]$, the function algebra of an infinite dimensional proalgebraic Poisson group $\widehat{H}$ whose tangent Lie bialgebra is $\hat{\mathfrak{h}}$ (cf. [BK]). All this can be seen as an application of (a "global version" of) the quantum duality principle: this claims (cf. [Dr], $\S 7$, or [CP], $\S 6$; see also [Ga3] for a proof) that the quantization of a Lie bialgebra - via a quantum universal enveloping algebra (QUEA) — provides

AMS 1991 Mathematics Subject Classification, Primary 17B37, Secondary 81R50

Partially supported by a post-doc fellowship of the Consiglio Nazionale delle Ricerche (Italy)

Typeset by $\mathcal{A} \mathcal{M} \mathcal{S}-\mathrm{T}_{\mathrm{E}} \mathrm{X}$ 
also a quantization of the dual Lie bialgebra (through its associated formal Poisson group) - via a quantum formal series Hopf algebra (QFSHA) — and, conversely, a QFSHA which quantizes a Lie bialgebra (via its associated formal Poisson group) yields a QUEA for the dual Lie bialgebra as well. In addition, both $\mathfrak{U}(\hat{\mathfrak{g}})$ and $\mathcal{U}(\hat{\mathfrak{g}})$ can be specialized at roots of 1 , and special quantum Frobenius morphisms $\mathfrak{U}_{\varepsilon}(\hat{\mathfrak{g}}) \longrightarrow \mathfrak{U}_{1}(\hat{\mathfrak{g}})$ and $\mathcal{U}_{1}(\hat{\mathfrak{g}}) \longleftrightarrow \mathcal{U}_{\varepsilon}(\hat{\mathfrak{g}})$ exist which are quantum analogues (in characteristic zero!) of the Frobenius morphisms $U\left(\hat{\mathfrak{g}}_{\mathbb{Z}_{p}}\right) \longrightarrow U\left(\hat{\mathfrak{g}}_{\mathbb{Z}_{p}}\right)$ and $F\left[\widehat{H}_{\mathbb{Z}_{p}}\right] \hookrightarrow F\left[\widehat{H}_{\mathbb{Z}_{p}}\right]$ which exist in characteristic $p$. Such results are not predicted by the quantum duality principle, and are typical of the Jimbo-Lusztig's approach to quantum groups.

Our aim is to find an analogue of $U_{q}(\hat{\mathfrak{g}})$ for the algebra $\hat{\mathfrak{h}}$ instead of $\hat{\mathfrak{g}}$; inspired by the quantum duality principle, and encouraged by the finite-type case (cf. [Ga1]), we choose as a reasonable candidate the linear dual $U_{q}(\hat{\mathfrak{g}})^{*}$, which has a natural structure of formal Hopf algebra. This dual can be studied by dualizing Drinfel'd's construction of the quantum double and using Tanisaki's pairings between quantum Borel (sub)algebras. So we find a description of $U_{q}(\hat{\mathfrak{g}})^{*}$, as a topological algebra with formal Hopf algebra structure, in terms of generators and relations: we call this algebra $U_{q}(\hat{\mathfrak{h}})$, for in fact we prove that it is for $\hat{\mathfrak{h}}$ what $U_{q}(\hat{\mathfrak{g}})$ is for $\hat{\mathfrak{g}}$. In particular, $U_{q}(\hat{\mathfrak{h}})$ has an integer form $\mathfrak{U}(\hat{\mathfrak{h}})$ (over $R$ ) which is a quantization of $U(\hat{\mathfrak{h}})$; moreover, $U_{q}(\hat{\mathfrak{h}})$ has also a second integer form $\mathcal{U}(\hat{\mathfrak{h}})$ which is a quantization of $F^{\infty}[\widehat{G}]$, where $\widehat{G}$ of course is a Kac-Moody Poisson group with $\hat{\mathfrak{g}}$ as tangent Lie bialgebra. More in general, both $\mathfrak{U}(\hat{\mathfrak{h}})$ and $\mathcal{U}(\hat{\mathfrak{h}})$ can be specialized at roots of 1 , and quantum Frobenius morphisms exist (for both kind of forms), which are dual of those of $U_{q}(\hat{\mathfrak{g}})$ and have a similar description.

Finally, a brief sketch of the main ideas of the paper. First, since $U_{q}(\hat{\mathfrak{g}})$ is a quotient of a quantum double $D_{q}(\hat{\mathfrak{g}}):=D\left(U_{q}\left(\hat{\mathfrak{b}}_{-}\right), U_{q}\left(\hat{\mathfrak{b}}_{+}\right), \pi\right)$, its linear dual $U_{q}(\hat{\mathfrak{g}})^{*}$ embeds into $D_{q}(\hat{\mathfrak{g}})^{*}$. Second, since $D_{q}(\hat{\mathfrak{g}}) \cong U_{q}\left(\hat{\mathfrak{b}}_{+}\right) \otimes U_{q}\left(\hat{\mathfrak{b}}_{-}\right)$(as coalgebras) we have $D_{q}(\hat{\mathfrak{g}})^{*} \cong U_{q}\left(\hat{\mathfrak{b}}_{+}\right)^{*} \widehat{\otimes} U_{q}\left(\hat{\mathfrak{b}}_{-}\right)^{*}$ (as algebras), where $\widehat{\otimes}$ denotes topological tensor product. Third, since quantum Borel algebras of opposite sign are perfectly paired, their linear duals are suitable completions of quantum Borel algebras of opposite sign: thus we find a presentation of $U_{q}(\hat{\mathfrak{g}})^{*}$ (as a topological algebra) by generators and relations which leads us to define $U_{q}(\hat{\mathfrak{h}}):=U_{q}(\hat{\mathfrak{g}})^{*}$ (actually, one has to keep track of some choice of lattices too, involved in the toral parts). From this, all claimed results follow. In particular, the form $\mathcal{U}(\hat{\mathfrak{h}})$ is the subset (of $U_{q}(\hat{\mathfrak{h}}):=U_{q}(\hat{\mathfrak{g}})^{*}$ ) of linear functions on $U_{q}(\hat{\mathfrak{g}})$ which are $R$-valued on $\mathfrak{U}(\hat{\mathfrak{g}})$, so $\mathcal{U}(\hat{\mathfrak{h}}) \cong H_{R}(\mathfrak{U}(\hat{\mathfrak{g}}), R)$ whence all results about specialisations of $\mathcal{U}(\hat{\mathfrak{h}})$ and its quantum Frobenius morphisms follow from those about $\mathfrak{U}(\hat{\mathfrak{g}})$. On the other hand, the form $\mathfrak{U}(\hat{\mathfrak{h}})$ is a proper subset of $\operatorname{Hom}_{R}(\mathcal{U}(\hat{\mathfrak{g}}), R)$, for sort of a (non-trivial) "locality condition" is required for elements of $\operatorname{Hom}_{R}(\mathcal{U}(\hat{\mathfrak{g}}), R)$ to belong to $\mathfrak{U}(\hat{\mathfrak{h}})$.

\section{ACKNOWLEDGEMENTS}

The author wishes to thank M. Rosso and E. Strickland for many helpful discussions, 
and I. Damiani and J. Beck for explanations about their papers. This article was partly prepared during a one-year stay of the author at the Institut de Recherche Mathématique Avancée of Strasbourg (France): the author takes this opportunity to thank all the colleagues and the staff of I.R.M.A. for the warm hospitality.

\section{$\S 1$ The classical objects}

1.1 Cartan data. Let $\mathfrak{g}$ be a simple finite dimensional complex Lie algebra, and consider the following data.

We take $I_{0}=\{1, \ldots, n\}$ to be the set of vertices of the Dynkin diagram of $\mathfrak{g}$ (see [Bo] for the identification between $I_{0}$ and $\left.\{1, \ldots, n\}\right) ; A_{0}=\left(a_{i j}\right)_{i, j \in I_{0}}$ the Cartan matrix of $\mathfrak{g} ; D_{0}=\operatorname{diag}\left(d_{1}, \ldots, d_{n}\right)$ the (unique) diagonal matrix with relatively prime positive integral entries such that $D_{0} A_{0}$ is symmetric; $\mathfrak{t}$, a Cartan subalgebra of $\mathfrak{g}$, with a fixed basis $\left\{h_{1}, \ldots, h_{n}\right\}=\left\{h_{i} \mid i \in I_{0}\right\} ; \Phi_{0}=\Phi_{0,+} \cup\left(-\Phi_{0,+}\right) \subseteq \mathfrak{t}^{*}$ the root system of $\mathfrak{g}$, with $\Phi_{0,+}$ the set of positive roots, and $\Pi:=\left\{\alpha_{1}, \ldots, \alpha_{n}\right\}=\left\{\alpha_{i} \mid i \in\right.$ $\left.I_{0}\right\}$ the set of simple roots; $Q_{0}:=\sum_{\alpha \in \Phi_{0}} \mathbb{Z} \alpha=\oplus_{i \in I_{0}} \mathbb{Z} \alpha_{i}$ the root lattice of $\mathfrak{g}$, and $Q_{0}^{\vee}:=\sum_{\alpha \in \Phi_{0}} \mathbb{Z} \alpha^{\vee}=\oplus_{i \in I_{0}} \mathbb{Z} \alpha_{i}^{\vee}$ the coroot lattice; $W_{0}$ the Weyl group of $\mathfrak{g}$. Finally, we fix a function $o: I_{0} \longrightarrow\{ \pm 1\}$ such that $a_{i j}<0 \Longrightarrow o(i) o(j)=-1$.

We denote $\hat{\mathfrak{g}}$ the untwisted affine Kac-Moody algebra associated to $\mathfrak{g}$ and we consider its loop-algebra like realization as $\hat{\mathfrak{g}}=\mathfrak{g} \otimes_{\mathbb{C}} \mathbb{C}\left[t, t^{-1}\right] \oplus \mathbb{C} \cdot c \oplus \mathbb{C} \cdot \partial$ with the Lie bracket given by: $[c, z]=0,\left[\partial, x \otimes t^{m}\right]=m x \otimes t^{m},\left[x \otimes t^{r}, y \otimes t^{s}\right]=[x, y] \otimes t^{r+s}+$ $\delta_{r,-s} r(x, y) c$ for all $z \in \hat{\mathfrak{g}}, x, y \in \mathfrak{g}, m, r, s \in \mathbb{Z}$ where $(\cdot, \cdot)$ is the Killing form of $\mathfrak{g}$, normalized in such a way that $\left(h_{i}, h_{j}\right)=\frac{a_{i j}}{d_{j}}$.

For $\hat{\mathfrak{g}}$ we define: $I:=\{0,1, \ldots, n\} \supset I_{0}$ to be the set of vertices of the Dynkin diagram, and $I_{\infty}:=I \cup\{\infty\} ; A=\left(a_{i j}\right)_{i, j \in I}$ the (generalized) Cartan matrix and $D=\operatorname{diag}\left(d_{0}, d_{1}, \ldots, d_{n}\right)$ with $d_{0}=1$ (so that $D A$ is symmetric); $\hat{\mathfrak{t}}:=\mathfrak{t} \oplus \mathbb{C} \cdot c \oplus \mathbb{C} \cdot \partial(\subseteq$ $\hat{\mathfrak{g}}) ; \Phi=\Phi_{+} \cup\left(-\Phi_{+}\right)\left(\subset(\mathfrak{t} \oplus \mathbb{C} \cdot c)^{*} \subset \hat{\mathfrak{t}}^{*}\right)$ the root system, $\Phi_{+}=\Phi_{+}^{\mathrm{re}} \cup \Phi_{+}^{\mathrm{im}}$ the set of positive roots, $\left\{\alpha_{0}, \alpha_{1}, \ldots, \alpha_{n}\right\}=\left\{\alpha_{i} \mid i \in I\right\}$ the set of simple roots, $\Phi_{+}^{\mathrm{im}}=$ $\left\{m \delta \mid m \in \mathbb{N}_{+}\right\}$the set of imaginary positive roots (where $\delta=\sum_{i \in I} d_{i} \alpha_{i}=\theta+\alpha_{0}$ and $\theta$ is the longest positive root of $\mathfrak{g}), \Phi_{+}^{\mathrm{re}}=\Phi_{0,+} \cup\left\{\alpha+m \delta \mid \alpha \in \Phi_{0}, m>0\right\}$ the set of real positive roots. Then $\hat{\mathfrak{g}}$ has a decomposition into direct sum of $\hat{\mathfrak{t}}$ and root spaces $\hat{\mathfrak{g}}=\hat{\mathfrak{t}} \oplus\left(\oplus_{\alpha \in \Phi} \hat{\mathfrak{g}}_{\alpha}\right)$, and $\operatorname{dim}_{\mathbb{C}}\left(\hat{\mathfrak{g}}_{\alpha}\right)=1$ if $\alpha \in \Phi^{\mathrm{re}}, \operatorname{dim}_{\mathbb{C}}\left(\hat{\mathfrak{g}}_{\alpha}\right)=\#\left(I_{0}\right)=n$ if $\alpha \in \Phi_{+}^{\mathrm{im}}$; therefore we define the set $\widetilde{\Phi}_{+}$of "positive roots with multiplicity" as $\widetilde{\Phi}_{+}:=\Phi_{+}^{\mathrm{re}} \cup \widetilde{\Phi}_{+}^{\mathrm{im}}$, where $\widetilde{\Phi}_{+}^{\mathrm{im}}:=\Phi_{+}^{\mathrm{im}} \times I_{0}$; then we denote $p: \widetilde{\Phi}_{+} \rightarrow \Phi_{+}$the natural projection map.

Furthermore, we have: the root lattice (of $\hat{\mathfrak{g}}$ ) $Q=\sum_{\alpha \in \Phi} \mathbb{Z} \cdot \alpha=\oplus_{i \in I} \mathbb{Z} \cdot \alpha_{i}=\mathbb{Z} \cdot \alpha_{0} \oplus$ $Q_{0}=Q_{0} \oplus \mathbb{Z} \cdot \delta, Q_{\infty}:=Q \oplus \mathbb{Z} \cdot \alpha_{\infty}$ (where $\alpha_{\infty} \in \hat{\mathfrak{t}}^{*}$ is defined by $\left\langle\alpha_{\infty}, \mathfrak{t}\right\rangle=0,\left\langle\alpha_{\infty}, \partial\right\rangle=$ $\left.0,\left\langle\alpha_{\infty}, c\right\rangle=1\right)$, and the order relation $\leq$ on $Q_{\infty}$ given by $\alpha \leq \beta \Longleftrightarrow \beta-\alpha \in Q_{+}$, with $Q_{+}:=\sum_{i \in I} \mathbb{N} \cdot \alpha_{i}$; the extended Cartan matrix $A_{\infty}=\left(a_{i j}\right)_{i, j \in I_{\infty}}$, defined by 
setting $a_{i \infty}:=\delta_{i, 0}, a_{\infty j}:=\delta_{0, j}$; the diagonal matrix $D_{\infty}=\operatorname{diag}\left(d_{0}, d_{1}, \ldots, d_{n}, d_{\infty}\right)$ with $d_{\infty}=1$; the non-degenerate symmetric bilinear form on $Q_{\infty} \otimes_{\mathbb{Z}} \mathbb{R}$ given by $\left(\alpha_{i}, \alpha_{j}\right)=d_{i} a_{i j}\left(\forall i, j \in I_{\infty}\right)$; the group $W=W_{0} \ltimes Q_{0}^{\vee}$, the subset of simple reflections $\left\{s_{0}, s_{1}, \ldots, s_{n}\right\}=\left\{s_{i} \mid i \in I\right\}(\subseteq W)$, and the length function $l: W \rightarrow \mathbb{N}$; the braid group $\mathcal{B}$ (associated to $W$ ), generated by $\left\{T_{0}, T_{1}, \ldots, T_{n}\right\}=\left\{T_{i} \mid i \in I\right\}$, and the section $T: W \rightarrow \mathcal{B}$ such that $T_{w}=T_{i_{1}} \cdots T_{i_{r}}$ for all $w=s_{i_{1}} \cdots s_{i_{r}} \in W$ with $l(w)=r$. Notice that the form $(\cdot, \cdot)$ is $W$-invariant. Finally, set $Q_{-}:=-Q_{+}=\sum_{i \in I}(-\mathbb{N}) \cdot \alpha_{i}$.

We define the weight lattice $P_{\infty}:=\operatorname{Hom}_{\mathbb{Z}}\left(Q_{\infty}, \mathbb{Z}\right)$ to be the dual lattice of $Q_{\infty}$, and we fix the $\mathbb{Z}$-basis $\left\{\omega_{i} \mid i \in I_{\infty}\right\}$ such that $\left\langle\alpha_{i} \mid \omega_{j}\right\rangle=\delta_{i j}$, where $\langle\mid\rangle: Q_{\infty} \times P_{\infty} \rightarrow \mathbb{Z}$ is the natural pairing; we set $P_{\infty}^{+}:=\sum_{i=1}^{n} \mathbb{N} \omega_{i}$ (the subset of dominant integral weights). Remark that $W$ acts on $Q_{\infty}$ too. Via the form $(\cdot, \cdot)$ we can embed $Q_{\infty}$ into $P_{\infty}$, so that $\alpha_{i}=\sum_{j \in I_{\infty}} d_{i} a_{i j} \omega_{j}$ for all $i \in I_{\infty}$. We extend the form $(\cdot, \cdot): Q_{\infty} \times Q_{\infty} \rightarrow \mathbb{Z}$ to a (non-degenerate symmetric) pairing $(\cdot, \cdot)$ : $\mathbb{Q} Q_{\infty} \times \mathbb{Q} Q_{\infty} \rightarrow \mathbb{Q}$ of $\mathbb{Q}$-vector spaces by scalar extension (hereafter $\mathbb{Q} T_{\infty}:=\mathbb{Q} \otimes_{\mathbb{Z}} T_{\infty}(T=Q, P)$ ): then restriction gives a pairing $(\cdot, \cdot): P_{\infty} \times P_{\infty} \rightarrow \mathbb{Q}$ (looking at $P_{\infty}$ as a sublattice of $\mathbb{Q} P_{\infty}=\mathbb{Q} Q_{\infty}$ ), which extends $(\cdot, \cdot): Q_{\infty} \times P_{\infty} \rightarrow \mathbb{Z}$ and takes values in $\mathbb{Z}\left[\Delta_{\infty}^{-1}\right]$, where $\Delta_{\infty}:=$ $\operatorname{det}\left(\left(a_{i j}\right)_{i, j \in I_{\infty}}\right)$. Finally we define $d_{\alpha}:=\frac{(\alpha, \alpha)}{2}$ for all $\alpha \in \Phi_{+}^{\mathrm{re}}$.

Given any pair of lattices $\left(M, M^{\prime}\right)$, with $Q_{\infty} \leq M, M^{\prime} \leq \mathbb{Q} P_{\infty}$, we say that they are dual of each other if $M^{\prime}=\left\{y \in \mathbb{Q} P_{\infty} \mid\langle M, y\rangle \subseteq \mathbb{Z}\right\}, M=\left\{x \in \mathbb{Q} P_{\infty} \mid\left\langle x, M^{\prime}\right\rangle \subseteq \mathbb{Z}\right\}$, the two conditions being equivalent; then for any lattice $M$ with $Q_{\infty} \leq M \leq \mathbb{Q} P_{\infty}$ there exists a unique dual lattice $M^{\prime}$ such that $Q_{\infty} \leq M^{\prime} \leq \mathbb{Q} P_{\infty}$ and $(\cdot, \cdot): \mathbb{Q} P_{\infty} \times$ $\mathbb{Q} P_{\infty} \rightarrow \mathbb{Q}$ restricts to a perfect pairing $(\cdot, \cdot): M \times M^{\prime} \rightarrow \mathbb{Z} ;$ in particular $P_{\infty}^{\prime}=Q_{\infty}$ and $Q_{\infty}^{\prime}=P_{\infty}$. In the sequel we denote by $\left\{\mu_{i} \mid i \in I_{\infty}\right\}$ and $\left\{\nu_{i} \mid i \in I_{\infty}\right\}$ fixed $\mathbb{Z}$-bases of $M$ and $M^{\prime}$ dual of each other, i. e. such that $\left(\mu_{i} \mid \nu_{j}\right)=\delta_{i j}$ for all $i, j \in I_{\infty}$, and we set $M_{+}:=M \cap P_{\infty}^{+}$. In the following our constructions will work in general for the pairs of dual lattices $\left(P_{\infty}, Q_{\infty}\right)$ and $\left(Q_{\infty}, P_{\infty}\right)$; but in the simply laced case (in which $\langle\rangle=,()),\left(M, M^{\prime}\right)$ will be any pair of dual lattices.

1.2 The classical Manin triple. Let be given for $\hat{\mathfrak{g}}$ the usual presentation by Chevalley-type generators $f_{i}, h_{j}, e_{i}\left(i \in I, j \in I_{\infty}\right)$ and relations; then let $\hat{\mathfrak{n}}_{+}$, resp. $\hat{\mathfrak{n}}_{-}$, be the Lie subalgebra of $\hat{\mathfrak{g}}$ generated by the $e_{i}$ 's, resp. the $f_{i}$ 's $(i \in I)$. Now let $\hat{\mathfrak{k}}$ be the Lie algebra $\hat{\mathfrak{k}}:=\hat{\mathfrak{g}} \oplus \hat{\mathfrak{g}}$; inside it we find a diagonal copy of $\hat{\mathfrak{g}}$ and a second Lie algebra

$$
\hat{\mathfrak{h}}:=\left\{\left(n_{-} t_{-}, t_{+} n_{+}\right) \mid n_{ \pm} \in \hat{\mathfrak{n}}_{ \pm}, t_{ \pm} \in \hat{\mathfrak{t}}, t_{-}+t_{+}=0\right\} \quad\left(\leq \hat{\mathfrak{b}}_{-} \times \hat{\mathfrak{b}}_{+} \leq \hat{\mathfrak{k}}\right)
$$

in particular $\hat{\mathfrak{h}}=\hat{\mathfrak{n}}_{-} \oplus \hat{\mathfrak{t}} \oplus \hat{\mathfrak{n}}_{+}$as vector spaces. Define a bilinear form on $\hat{\mathfrak{k}}$ by the formula $\left\langle x_{1} \oplus y_{1}, x_{2} \oplus y_{2}\right\rangle:=\frac{1}{2}\left(y_{1}, y_{2}\right)-\frac{1}{2}\left(x_{1}, x_{2}\right)$, where $(\cdot, \cdot)$ is the Killing form; this form makes $(\hat{\mathfrak{k}}, \hat{\mathfrak{g}}, \hat{\mathfrak{h}})$ into a Manin triple; in particular $\hat{\mathfrak{g}}$ and $\hat{\mathfrak{h}}$ are Lie bialgebras, and the bilinear form on $\hat{\mathfrak{k}}$ gives by restriction a non-degenerate pairing $\langle\rangle:, \hat{\mathfrak{h}} \otimes \hat{\mathfrak{g}} \rightarrow \mathbb{C}$ which respect the Lie bialgebra structure on both sides, that is $\left\langle x,\left[y_{1}, y_{2}\right]\right\rangle=\left\langle\delta_{\hat{\mathfrak{h}}}(x), y_{1} \otimes y_{2}\right\rangle$, $\left\langle\left[x_{1}, x_{2}\right], y\right\rangle=\left\langle x_{1} \otimes x_{2}, \delta_{\hat{\mathfrak{g}}}(y)\right\rangle$, where $\delta$ is the Lie cobracket. 
1.3 The Poisson Hopf coalgebra $U(\hat{\mathfrak{h}})$. The presentation of $\hat{\mathfrak{g}}$ by generators and relations gives a similar one for $U(\hat{\mathfrak{g}})$, with the same generators. From the latter we take for $U(\hat{\mathfrak{h}})$ the following presentation, where $\mathrm{f}_{i}=f_{i} \oplus 0, \mathrm{~h}_{i}=h_{i} \oplus h_{i}, \mathrm{e}_{i}=0 \oplus e_{i}$ : $U(\hat{\mathfrak{h}})$ is the associative $\mathbb{C}$-algebra with 1 generated by $\mathrm{f}_{i}, \mathrm{~h}_{i}, \mathrm{e}_{i}\left(i=\in I_{\infty}\right)$ with relations

$$
\begin{gathered}
\mathrm{h}_{r} \mathrm{~h}_{s}-\mathrm{h}_{s} \mathrm{~h}_{r}=0, \quad \mathrm{e}_{i} \mathrm{f}_{j}-\mathrm{f}_{j} \mathrm{e}_{i}=0, \quad \mathrm{~h}_{r} \mathrm{f}_{j}-\mathrm{f}_{j} \mathrm{~h}_{r}=a_{r j} \mathrm{f}_{j}, \quad \mathrm{~h}_{r} \mathrm{e}_{j}-\mathrm{e}_{j} \mathrm{~h}_{r}=a_{r j} \mathrm{e}_{j} \\
\sum_{k=0}^{1-a_{i j}}(-1)^{k}\left(\begin{array}{c}
1-a_{i j} \\
k
\end{array}\right) \mathrm{f}_{i}^{1-a_{i j}-k} \mathrm{f}_{j} \mathrm{f}_{i}^{k}=0, \sum_{k=0}^{1-a_{i j}}(-1)^{k}\left(\begin{array}{c}
1-a_{i j} \\
k
\end{array}\right) \mathrm{e}_{i}^{1-a_{i j}-k} \mathrm{e}_{j} \mathrm{e}_{i}^{k}=0
\end{gathered}
$$

for $r, s \in I_{\infty}, i, j \in I(i \neq j$ in the bottom row $)$; its Hopf structure is given by

$$
\Delta(x)=x \otimes 1+1 \otimes x, S(x)=-x, \epsilon(x)=0 \quad \forall x \in\left\{\mathrm{f}_{i}, \mathrm{~h}_{j}, \mathrm{e}_{i} \mid i \in I, j \in I_{\infty}\right\}
$$

(the natural one) and the co-Poisson structure $\delta=\delta_{\hat{\mathfrak{h}}}: U(\hat{\mathfrak{h}}) \rightarrow U(\hat{\mathfrak{h}}) \otimes U(\hat{\mathfrak{h}})$ by

$$
\begin{aligned}
& \delta\left(\mathrm{f}_{i}\right)=d_{i} \cdot\left(\mathrm{h}_{i} \otimes \mathrm{f}_{i}-\mathrm{f}_{i} \otimes \mathrm{h}_{i}\right)+2 d_{i}^{-1} \cdot \sum_{\substack{\alpha, \beta \in \widetilde{\Phi}_{+} \\
p(\alpha)-p(\beta)=-\alpha_{i}}} c_{\alpha, \beta}^{i,+} d_{\alpha} d_{\beta} \cdot\left(\mathrm{e}_{\alpha} \otimes \mathrm{f}_{\beta}-\mathrm{f}_{\beta} \otimes \mathrm{e}_{\alpha}\right) \\
& \delta\left(\mathrm{h}_{i}\right)=4 d_{i}^{-1} \cdot \sum_{\substack{\alpha, \beta \in \widetilde{\Phi}_{+} \\
p(\alpha)-p(\beta)=0}} c_{\alpha, \beta} d_{\alpha}\left(\alpha_{i} \mid p(\alpha)\right) \cdot\left(\mathrm{e}_{\alpha} \otimes \mathrm{f}_{\beta}-\mathrm{f}_{\beta} \otimes \mathrm{e}_{\alpha}\right) \\
& \delta\left(\mathrm{e}_{i}\right)=d_{i} \cdot\left(\mathrm{e}_{i} \otimes \mathrm{h}_{i}-\mathrm{h}_{i} \otimes \mathrm{e}_{i}\right)+2 d_{i}^{-1} \cdot \sum_{\substack{\alpha, \beta \in \widetilde{\Phi}_{+} \\
p(\alpha)-p(\beta)=+\alpha_{i}}} c_{\alpha, \beta}^{i,-} d_{\alpha} d_{\beta} \cdot\left(\mathrm{e}_{\alpha} \otimes \mathrm{f}_{\beta}-\mathrm{f}_{\beta} \otimes \mathrm{e}_{\alpha}\right)
\end{aligned}
$$

for all $i \in I, j \in I_{\infty}$; here the $\mathrm{e}_{\gamma}$ 's and the $\mathrm{f}_{\gamma}$ 's are suitable "root-with-multiplicity vectors" (respectively of "weight" $p(\gamma)$ and $-p(\gamma)$ ) such that $\left\langle\mathrm{e}_{\gamma}, f_{\eta}\right\rangle=+\delta_{\gamma, \eta} d_{\gamma} / 2$, $\left\langle\mathrm{f}_{\gamma}, f_{\eta}\right\rangle=-\delta_{\gamma, \eta} d_{\gamma} / 2\left(f_{\eta}\right.$ and $e_{\eta}$ being root-with-multiplicity vectors of $\left.\hat{\mathfrak{g}}\right)$, the $c_{\alpha, \beta}$ 's are given by the equations $\left[e_{\beta}, f_{\alpha}\right]=c_{\alpha, \beta} h_{p(\alpha)}$ and the $c_{\alpha, \beta}^{i, \pm}$ 's by $\left[f_{\alpha}, e_{\beta}\right]=c_{\alpha, \beta}^{i,-} \cdot f_{i}$, $\left[f_{\alpha}, e_{\beta}\right]=c_{\alpha, \beta}^{i,+} \cdot e_{i}$. Note that the formulas above contains infinite sums, so $\delta$ in fact

takes values in a certain completion of $U(\hat{\mathfrak{h}}) \otimes U(\hat{\mathfrak{h}})$; hence to be precise $U(\hat{\mathfrak{h}})$ is a Hopf algebra which is co-Poisson only in a larger sense; and similarly for $\hat{\mathfrak{h}}$ as a Lie bialgebra).

\section{$\S 2$ Quantum Borel algebras and DRT pairings}

2.1 Quantum Borel algebras. From now on $M$ will be any lattice such that $Q_{\infty} \leq M \leq P_{\infty}$; then $M^{\prime}$ will be the dual lattice, as in $\S 1.1$ (upon conditions therein). 
For all $s, n \in \mathbb{N}$, let $(n)_{q}:=\frac{q^{n}-1}{q-1}(\in \mathbb{Z}[q]),(n)_{q} !:=\prod_{r=1}^{n}(r)_{q},\left(\begin{array}{c}n \\ s\end{array}\right)_{q}:=\frac{(n)_{q} !}{(s)_{q} !(n-s)_{q} !}$ $(\in \mathbb{Z}[q])$, and $[n]_{q}:=\frac{q^{n}-q^{-n}}{q-q^{-1}}\left(\in \mathbb{Z}\left[q, q^{-1}\right]\right),[n]_{q} !:=\prod_{r=1}^{n}[r]_{q},\left[\begin{array}{l}n \\ s\end{array}\right]_{q}:=\frac{[n]_{q} !}{[s]_{q} ![n-s]_{q} !}(\in$ $\left.\mathbb{Z}\left[q, q^{-1}\right]\right)$. Let $q_{\alpha}:=q^{d_{\alpha}}$ for all $\alpha \in \Phi_{+}^{\text {re }}$, and $q_{i}:=q_{\alpha_{i}}$ for all $i \in I$; then set $q_{(r \delta, i)}:=q_{i}$ for every positive imaginary $\operatorname{root}(r \delta, i) \in \Phi_{+}^{\mathrm{im}}$.

We define $U_{q}^{M}\left(\hat{\mathfrak{b}}_{-}\right)$, resp. $U_{q}^{M}\left(\hat{\mathfrak{b}}_{+}\right)$, to be the associative $\mathbb{C}(q)$-algebra with 1 generated by $L_{\mu}(\mu \in M), F_{1}, \ldots, F_{n}$, resp. $L_{\mu}(\mu \in M), E_{1}, \ldots, E_{n}$, with relations

$$
\begin{array}{cc}
L_{0}=1, & L_{\mu} L_{\nu}=L_{\mu+\nu}, \\
L_{\mu} F_{j}=q^{-\left(\alpha_{j} \mid \mu\right)} F_{j} L_{\mu}, & \sum_{p+s=1-a_{i j}}(-1)^{s}\left[\begin{array}{c}
1-a_{i j} \\
s
\end{array}\right]_{q_{i}} F_{i}^{p} F_{j} F_{i}^{s}=0 \\
\operatorname{resp.} \quad L_{\mu} E_{j}=q^{\left(\alpha_{j} \mid \mu\right)} E_{j} L_{\mu}, & \sum_{p+s=1-a_{i j}}(-1)^{s}\left[\begin{array}{c}
1-a_{i j} \\
s
\end{array}\right]_{q_{i}} E_{i}^{p} E_{j} E_{i}^{s}=0
\end{array}
$$

for all $i, j \in I, i \neq j$, and $\mu, \nu \in M$; these are both Hopf algebras, with

$$
\begin{array}{ccc}
\Delta\left(F_{i}\right)=F_{i} \otimes L_{-\alpha_{i}}+1 \otimes F_{i}, & \epsilon\left(F_{i}\right)=0, & S\left(F_{i}\right)=-F_{i} L_{\alpha_{i}} \\
\Delta\left(L_{\mu}\right)=L_{\mu} \otimes L_{\mu}, & \epsilon\left(L_{\mu}\right)=1, & S\left(L_{\mu}\right)=L_{-\mu} \\
\Delta\left(E_{i}\right)=E_{i} \otimes 1+L_{\alpha_{i}} \otimes E_{i}, & \epsilon\left(E_{i}\right)=0, & S\left(E_{i}\right)=-L_{-\alpha_{i}} E_{i}
\end{array}
$$

for all $i \in I, \mu \in M$. We also consider the subalgebras $U_{q}^{M}(\mathfrak{t})$ (generated by the $L_{\mu}$ 's), $U_{q}\left(\mathfrak{n}_{-}\right)$(generated by the $F_{i}$ 's), $U_{q}\left(\mathfrak{n}_{+}\right)$(generated by the $E_{i}$ 's). In the sequel we shall use the notation $K_{\alpha}:=L_{\alpha}, M_{\mu}:=L_{\mu}, \Lambda_{\nu}:=L_{\nu}\left(\forall \alpha \in Q_{\infty}, \mu \in M, \nu \in M^{\prime}\right)$ (and in particular $\left.K_{i}:=K_{\alpha_{i}}, M_{i}:=M_{\mu_{i}} \Lambda_{i}:=\Lambda_{\nu_{i}}\right)$, and $U_{\leq}^{M}:=U_{q}^{M}\left(\hat{\mathfrak{b}}_{-}\right), U_{\geq}^{M}:=U_{q}^{M}\left(\hat{\mathfrak{b}}_{+}\right)$, $U_{0}^{M}:=U_{q}^{M}(\mathfrak{t}), U_{-}:=U_{q}\left(\mathfrak{n}_{-}\right), U_{+}:=U_{q}\left(\mathfrak{n}_{+}\right)$. Multiplication yields various linear isomorphisms, which we shall refer to as triangular decompositions, namely

$$
U_{\leq}^{M} \cong U_{-} \otimes U_{0}^{M} \cong U_{0}^{M} \otimes U_{-}, \quad U_{\leq}^{M} \cong U_{+} \otimes U_{0}^{M} \cong U_{0}^{M} \otimes U_{+}
$$

A natural $Q_{+}$-grading, resp. $Q_{-}$-grading, (of algebras) is defined on $U_{\geq}^{M}$, resp. $U_{\geq}^{M}$, by setting $\operatorname{deg}\left(E_{i}\right):=\alpha_{i}, \operatorname{deg}\left(F_{i}\right):=-\alpha_{i}(i \in I)$; then these are also gradings of Hopf algebras, inherited by the various subalgebras defined above.

2.2 DRT pairings. If $H$ is any Hopf algebra, we let $H^{o p}$ be the same coalgebra with opposite multiplication, and $H_{o p}$ the same algebra with opposite comultiplication.

There exists perfect (i. e. non-degenerate) pairings of graded Hopf algebras (cf. [Ta])

$$
\begin{aligned}
& \pi:\left(U_{\leq}^{M}\right)_{o p} \otimes U_{\geq}^{M^{\prime}} \longrightarrow \mathbb{C}(q), \pi: U_{\leq}^{M} \otimes\left(U_{\geq}^{M^{\prime}}\right)^{o p} \longrightarrow \mathbb{C}(q) \\
& \bar{\pi}:\left(U_{\geq}^{M}\right)_{o p} \otimes U_{\leq}^{M^{\prime}} \longrightarrow \mathbb{C}(q), \quad \bar{\pi}: U_{\geq}^{M} \otimes\left(U_{\leq}^{M^{\prime}}\right) \text { op } \longrightarrow \mathbb{C}(q) \\
& \pi\left(L_{\mu}, L_{\nu}\right)=q^{-(\mu, \nu)}, \quad \pi\left(L_{\mu}, E_{j}\right)=0, \pi\left(F_{i}, L_{\nu}\right)=0, \quad \pi\left(F_{i}, E_{j}\right)=-\delta_{i j}\left(q_{i}-q_{i}^{-1}\right)^{-1} \\
& \bar{\pi}\left(L_{\mu}, L_{\nu}\right)=q^{+(\mu, \nu)}, \bar{\pi}\left(E_{i}, L_{\nu}\right)=0, \bar{\pi}\left(L_{\mu}, F_{j}\right)=0, \quad \bar{\pi}\left(E_{i}, F_{j}\right)=+\delta_{i j}\left(q_{i}-q_{i}^{-1}\right)^{-1}
\end{aligned}
$$


These pairings were introduced by Drinfel'd, Rosso, Tanisaki, and others, so we shall call them DRT pairings. If $\pi$ is any DRT pairing we shall also set $\langle x, y\rangle_{\pi}$ for $\pi(x, y)$.

2.3 Quantum root vectors. We define quantum root vectors along the lines of Beck's work (cf. [Be1], [Be2]), but fixing conventions as in [Da], [Ga2]. It is possible to define a total order $\preceq$ on the set $\widetilde{\Phi}_{+}$such that $\beta_{1} \preceq \beta_{2} \preceq \beta_{3} \preceq \cdots \preceq \beta_{k-1} \preceq$ $\beta_{k} \preceq \beta_{k+1} \preceq \cdots \preceq((r+1) \delta, n) \preceq(r \delta, 1) \preceq(r \delta, 2) \cdots \preceq(2 \delta, n) \preceq(\delta, 1) \preceq(\delta, 2) \preceq$ $\cdots \preceq(\delta, n) \preceq \cdots \preceq \beta_{-(k+1)} \preceq \beta_{-k} \preceq \beta_{-(k-1)} \cdots \preceq \beta_{-2} \preceq \beta_{-1} \preceq \beta_{0}$ and moreover $\left\{\beta_{k} \mid k \geq 1\right\}=\left\{r \delta-\alpha \mid r>0, \alpha \in \Phi_{0,+}\right\}$ and $\left\{\beta_{k} \mid k \leq 0\right\}=\{r \delta+\alpha \mid r \geq 0, \alpha \in$ $\left.\Phi_{0,+}\right\}$. Let such an order be fixed: then one defines - as in [Ga2], §2.2 - (quantum root) vectors $E_{\gamma}$, for all $\gamma \in \widetilde{\Phi}_{+}$, and also root vectors $F_{\gamma}$ (for all $\gamma \in \widetilde{\Phi}_{+}$) associated to negative roots. Definitions give $E_{\gamma} \in\left(U_{+}\right)_{p(\gamma)}, F_{\gamma} \in\left(U_{-}\right)_{-p(\gamma)}$ for all $\gamma \in \widetilde{\Phi}_{+}$. For later use we recall an important property of imaginary root vectors:

Claim: All root vectors attached to imaginary roots commute with each other.

2.4 PBW bases and orthogonality. It is proved in [Be2] that the set $B_{+}$of ordered monomials in the root vectors $E_{\alpha}$ 's (according to the order $\preceq$ on $\widetilde{\Phi}_{+}$), namely the $\prod_{\alpha \in \Phi_{+}} E_{\alpha}^{n_{\alpha}}$ 's, is a $\mathbb{C}(q)$-basis of $U_{+}$(Remark: hereafter, when dealing with such a kind of monomials, or the like, the function $\alpha \mapsto n_{\alpha} \in \mathbb{N}$ will always be zero for almost all 1 $\alpha$ ). Similarly, the set $B_{-}$of ordered monomials in the root vectors $F_{\alpha}$ 's is a $\mathbb{C}(q)$-basis of $U_{-}$; in addition, the set $B_{0}^{M}:=\left\{K_{\alpha} \mid \alpha \in Q_{\infty}\right\} \equiv\left\{\prod_{i \in I_{\infty}} K_{i}^{l_{i}} \mid l_{i} \in \mathbb{Z} \forall i \in I_{\infty}\right\}$ is a $\mathbb{C}(q)$-basis of $U_{0}^{M}$. Then from triangular decompositions one concludes that the sets of ordered monomials $B_{+} \cdot B_{0}^{M}$ and $B_{0}^{M} \cdot B_{+}$are $\mathbb{C}(q)$-bases of $U_{+}$, and similarly $B_{-} \cdot B_{0}^{M}$ and $B_{0}^{M} \cdot B_{-}$are $\mathbb{C}(q)$-bases of $U_{-}$. From [Da] we know that

$$
\begin{gathered}
\pi\left(F_{\alpha}, E_{\beta}\right)=\frac{\delta_{\alpha, \beta}}{\left(q_{\alpha}^{-1}-q_{\alpha}\right)}, \pi\left(F_{\alpha}, E_{\gamma}\right)=0, \pi\left(F_{\gamma}, E_{\alpha}\right)=0 \quad \forall \alpha, \beta \in \Phi_{+}^{\mathrm{re}}, \gamma \in \widetilde{\Phi}_{+}^{\mathrm{im}} \\
\pi\left(F_{(r \delta, i)}, E_{(s \delta, j)}\right)=\delta_{r, s}(o(i) o(j))^{r} \frac{\left[r a_{i j}\right]_{q_{i}}}{r\left(q_{j}^{-1}-q_{j}\right)} \quad \forall(r, \delta, i),(s \delta, j) \in \widetilde{\Phi}_{+}^{\mathrm{im}}
\end{gathered}
$$

and similarly for $\bar{\pi}$, for we have $\bar{\pi}\left(E_{\alpha}, F_{\beta}\right)=-\pi\left(F_{\beta}, E_{\alpha}\right)$; these formulas are the starting point to build up orthogonal bases of $U_{-}$and $U_{+}$: the key result is the following.

Lemma 2.5 (cf. [Da], [Ga2]). For all $r \in \mathbb{N}_{+}$, let $V_{r}$, resp. $W_{r}$, be the $\mathbb{C}(q)$-vector space with basis $\left\{E_{(r \delta, i)} \mid i \in I_{0}\right\}$, resp. $\left\{F_{(r \delta, i)} \mid i \in I_{0}\right\}$, and let $\left\{x_{r, i} \mid i \in I_{0}\right\}$ and $\left\{y_{r, j} \mid j \in I_{0}\right\}$ be bases of $V_{r}$ and $W_{r}$ orthogonal of each other with respect to $\pi$, namely $\pi\left(y_{r, i}, x_{r, i}\right) \neq 0$ and $\pi\left(y_{r, j}, x_{r, i}\right)=0$ for all $i, j \in I_{0}$. Then $\left\{\prod_{k \leq 0} E_{\beta_{k}}^{n_{k}}\right.$. $\left.\prod_{r \in \mathbb{N}, i \in I_{0}} x_{r, i}^{n_{r, i}} \cdot \prod_{k>0} E_{\beta_{k}}^{n_{k}} \mid n_{k}, n_{r, i} \in \mathbb{N} \forall k, i\right\}$ and $\left\{\prod_{k \leq 0} F_{\beta_{k}}^{m_{k}} \cdot \prod_{r \in \mathbb{N}, j \in I_{0}} y_{s, j}^{m_{s, j}}\right.$. $\left.\prod_{k>0} F_{\beta_{k}}^{m_{k}} \mid m_{k}, m_{s, j} \in \mathbb{N} \forall k, j\right\}$ are bases of $U_{+}$and $U_{-}$which are orthogonal of each

\footnotetext{
${ }^{1}$ Hereafter by "almost all" we shall always mean "all but a finite number of".
} 
other. And similarly for the pairing $\bar{\pi}$. More precisely, we have

$$
\begin{gathered}
\pi\left(\prod_{k \leq 0} F_{\beta_{k}}^{m_{k}} \cdot \prod_{r \in \mathbb{N}_{+}, j \in I_{0}} y_{r, j}^{m_{r, j}} \cdot \prod_{k>0} F_{\beta_{k}}^{m_{k}}, \prod_{h \leq 0} E_{\beta_{h}}^{n_{h}} \cdot \prod_{s \in \mathbb{N}_{+}, i \in I_{0}} x_{s, i}^{n_{s, i}} \cdot \prod_{h>0} E_{\beta_{h}}^{n_{h}}\right)= \\
=\prod_{\alpha \in \Phi_{+}^{\mathrm{re}}} \delta_{n_{\alpha}, m_{\alpha}} q_{\alpha}^{\left(\begin{array}{c}
n_{\alpha} \\
2
\end{array}\right)} \frac{\left[n_{\alpha}\right]_{q_{\alpha}} !}{\left(q_{\alpha}^{-1}-q_{\alpha}\right)^{n_{\alpha}}} \cdot \prod_{r \in \mathbb{N}, i \in I_{0}} \delta_{n_{r, i}, m_{r, i}} n_{r, i} ! \pi\left(y_{r, i}, x_{r, i}\right)^{n_{r, i}} \\
\bar{\pi}\left(\prod_{k \leq 0} E_{\beta_{k}}^{n_{k}} \cdot \prod_{r \in \mathbb{N}_{+}, i \in I_{0}} x_{r, i}^{n_{r, i}} \cdot \prod_{k>0} E_{\beta_{k}}^{n_{k}}, \prod_{h \leq 0} F_{\beta_{h}}^{m_{h}} \cdot \prod_{s \in \mathbb{N}_{+}, j \in I_{0}} y_{s, j}^{m_{s, j}} \cdot \prod_{h>0} F_{\beta_{h}}^{m_{h}}\right)= \\
=\prod_{\alpha \in \Phi_{+}^{\mathrm{re}}} \delta_{n_{\alpha}, m_{\alpha}} q_{\alpha}^{\left(\begin{array}{c}
n_{\alpha} \\
2
\end{array}\right)} \frac{\left[n_{\alpha}\right]_{q_{\alpha}} !}{\left(q_{\alpha}-q_{\alpha}^{-1}\right)^{n_{\alpha}}} \cdot \prod_{r \in \mathbb{N}, i \in I_{0}} \delta_{n_{r, i}, m_{r, i}, i} n_{r, i} ! \pi\left(x_{r, i}, y_{r, i}\right)^{n_{r, i}} .
\end{gathered}
$$

2.6 Integer forms. Let $R$ be the subring of $\mathbb{C}(q)$ of all rational functions having no poles at roots of unity of odd order. Let $\mathfrak{U}_{\leq}^{M}$ be the $R$-subalgebra of $U_{\leq}^{M}$ generated by the elements $F_{i}^{(m)}:=F_{i}^{m} /[m]_{q_{i}} !,\left(\begin{array}{c}M_{j} ; c \\ t\end{array}\right):=\prod_{s=1}^{t} \frac{q_{j}^{c-s+1} M_{j}-1}{q_{j}^{s}-1}$ (the so-called $q_{-}^{-}$ divided powers) and $M_{j}^{-1}$ for all $m, c, t \in \mathbb{N}, i \in I, j \in I_{\infty}$. It is known (cf. [Lu2]) that $\mathfrak{U}_{\leq}^{M}$ is a Hopf subalgebra of $U_{\leq}^{M}$. It is proved in [Ga2] that $\mathfrak{U}_{\leq}^{M}$ has a PBW basis (as an $R_{-}^{-}$

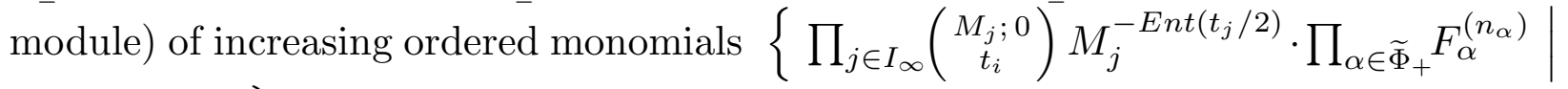
$\left.n_{\alpha} \in \mathbb{N}, t_{j} \in \mathbb{N}\right\}$ (recall that the $n_{\alpha}$ 's are almost all zero), where we use notation

$$
F_{\gamma}^{(n)}:=\frac{F_{\gamma}^{n}}{[n]_{q_{\gamma}} !} \quad \forall \gamma \in \Phi_{+}^{\mathrm{re}}, \quad F_{(r \delta, i)}^{(n)}:=\frac{\left(r /[r]_{q_{i}} \cdot F_{(r \delta, i)}\right)^{n}}{n !} \quad \forall(r \delta, i) \in \widetilde{\Phi}_{+}^{\mathrm{im}} ;
$$

a similar PBW basis of decreasing ordered monomials also exists; thus $\mathfrak{U}_{\leq}^{M}$ is an $R$-form of $U_{\leq}^{M}$. Similarly we define the Hopf subalgebra $\mathfrak{U}_{\geq}^{M}$ and find PBW bases for it.

Let $\bar{E}_{\alpha}:=\left(q_{\alpha}-q_{\alpha}^{-1}\right) E_{\alpha}$ for all $\alpha \in \widetilde{\Phi}_{+}$, and let $\mathcal{U}_{\geq}^{M}$ be the $R$-subalgebra of $U_{\geq}^{M}$ generated by all the $\bar{E}_{\alpha}$ 's and all the $M_{i}$ 's; then (cf. [BK]) $\mathcal{U}_{\geq}^{M}$ is a Hopf subalgebra of $U_{\geq}^{M}$, with a PBW basis (as an $R$-module) $\left\{\prod_{j \in I_{\infty}} M_{j}^{t_{j}} \cdot \prod_{\alpha \in \widetilde{\Phi}_{+}} \bar{E}_{\alpha}^{n_{\alpha}} \mid n_{\alpha} \in \mathbb{N}, t_{j} \in \mathbb{N}\right\}$ of increasing ordered monomials and a similar PBW basis of decreasing ordered monomials; in particular $\mathcal{U}_{\geq}^{M}$ is an $R$-form of $U_{\geq}^{M}$. The same procedure yields the definition of the Hopf subalgebra $\mathcal{U}_{\leq}^{M}$ and provides PBW bases for it.

Similar constructions and results hold for the algebras $U_{-}, U_{0}^{M}, U_{+}$, providing integer forms $\mathfrak{U}_{-}, \mathcal{U}_{+}$, and so on, all of them endowed with PBW bases of ordered "monomials". Finally, we have triangular decompositions

$$
\begin{gathered}
\mathfrak{U}_{\leq}^{M} \cong \mathfrak{U}_{-} \otimes \mathfrak{U}_{0}^{M} \cong \mathfrak{U}_{0}^{M} \otimes \mathfrak{U}_{-}, \mathfrak{U}_{\geq}^{M} \cong \mathfrak{U}_{+} \otimes \mathfrak{U}_{0}^{M} \cong \mathfrak{U}_{0}^{M} \otimes \mathfrak{U}_{+} \\
\mathcal{U}_{\leq}^{M} \cong \mathcal{U}_{-} \otimes \mathcal{U}_{0}^{M} \cong \mathcal{U}_{0}^{M} \otimes \mathcal{U}_{-}, \mathcal{U}_{\geq}^{M} \cong \mathcal{U}_{+} \otimes \mathcal{U}_{0}^{M} \cong \mathcal{U}_{0}^{M} \otimes \mathcal{U}_{+}
\end{gathered}
$$


2.7 $R$-duality among integer forms. For all $r \in \mathbb{N}_{+}$, let $V_{r}$ and $W_{r}$ be the $\mathbb{C}(q)$-vector spaces defined in Lemma 2.5. Consider the elements $\widehat{E}_{(r \delta, i)}:=\left(r /[r]_{q_{i}}\right)$. $E_{(r \delta, i)} \in V_{r}\left(i \in I_{0}\right)$; then $\left\{\widehat{E}_{(r \delta, i)} \mid i \in I_{0}\right\}$ is a basis of $V_{r}$. The basis of $W_{r}$ dual of $\left\{\widehat{E}_{(r \delta, i)} \mid i \in I_{0}\right\}$ with respect to $\pi$, is the subset $\left\{\dot{\bar{F}}_{(r \delta, j)} \mid j \in I_{0}\right\} \subset W_{r}$ such that $\pi\left(\dot{\bar{F}}_{(r \delta, j)}, \widehat{E}_{(r \delta, i)}\right)=\delta_{j i}$, for all $i, j \in I_{0}$. Similarly, we define the basis $\left\{\dot{\hat{F}}_{(r \delta, j)} \mid j \in\right.$ $\left.I_{0}\right\}$ of $W_{r}$ dual of $\left\{\bar{E}_{(r \delta, i)} \mid i \in I_{0}\right\}$ with respect to $\pi$. Similar definitions hold with $\bar{\pi}$ instead of $\pi$, and reversing the roles of $E$ and $F$.

Definitions and formulas in $\S 2.4$ give the following (see [Ga2], Proposition 4.6):

Claim (a): for all $r \in \mathbb{N}_{+}$, the basis $\left\{\dot{\bar{F}}_{(r \delta, j)} \mid j \in I_{0}\right\} \subset W_{r}$ of $W_{r}$ dual of $\left\{\widehat{E}_{(r \delta, i)} \mid i \in I_{0}\right\}$ (with respect to $\pi$ or to $\bar{\pi}$ ) lies in the $R$-linear span of $\left\{\bar{F}_{(r \delta, j)} \mid j \in\right.$ $\left.I_{0}\right\} \subset W_{r}$ : in particular, it lies in $\mathcal{U}_{-}$. Similarly, the basis $\left\{\dot{\widehat{F}}_{(r \delta, j)} \mid j \in I_{0}\right\} \subset W_{r}$ of $W_{r}$ dual of $\left\{\bar{E}_{(r \delta, i)} \mid i \in I_{0}\right\}$ (with respect to $\pi$ or to $\bar{\pi}$ ) lies in the $R$-linear span of $\left\{\widehat{F}_{(r \delta, j)} \mid j \in I_{0}\right\} \subset W_{r}$ : in particular, it lies in $\mathfrak{U}_{-}$. Similar statements hold when reversing the roles of $E$ and $F$.

Then Lemma 2.5, and results in [DL], $§ 3$ (see also Lemma 4.4 later on) give:

Claim (b): If in one integer form (of a subalgebra of a quantum Borel algebra) we fix a $\overline{P B W}$ basis, then on the other hand the form (of the opposite algebra) of opposite typographic "font" does contain a PBW basis - made with "dotted" root vectors (which are equal to the old ones for real roots) - which is dual to the initial one.

Therefore integer forms of opposite "fonts" are $R$-dual of each other, in the following sense: for every DRT pairing, if we take $\mathfrak{U}$ on one side, then the form $\mathcal{U}$ on the other side is equal to the subset of all elements which paired with $\mathfrak{U}$ give a value in $R$; and similarly exchanging $\mathfrak{U}$ and $\mathcal{U}$. For instance

$$
\begin{aligned}
\mathfrak{U}_{0}^{M}=\left\{y \in U_{0}^{M} \mid \pi\left(\mathcal{U}_{0}^{M^{\prime}}, y\right) \subseteq R\right\} & =\left\{x \in U_{0}^{M} \mid \bar{\pi}\left(x, \mathcal{U}_{0}^{M^{\prime}}\right) \subseteq R\right\} \\
\mathcal{U}_{0}^{M}=\left\{y \in U_{0}^{M} \mid \pi\left(\mathfrak{U}_{0}^{M^{\prime}}, y\right) \subseteq R\right\} & =\left\{x \in U_{0}^{M} \mid \bar{\pi}\left(x, \mathfrak{U}_{0}^{M^{\prime}}\right) \subseteq R\right\} \\
\mathfrak{U}_{-}=\left\{x \in U_{-} \mid \pi\left(x, \mathcal{U}_{+}\right) \subseteq R\right\} & =\left\{y \in U_{-} \mid \bar{\pi}\left(\mathcal{U}_{+}, y\right) \subseteq R\right\} \\
\mathcal{U}_{\geq}^{M}=\left\{x \in U_{\geq}^{M} \mid \bar{\pi}\left(x, \mathfrak{U}_{\leq}^{M^{\prime}}\right) \subseteq R\right\} & =\left\{y \in U_{\geq}^{M} \mid \pi\left(\mathfrak{U}_{\leq}^{M^{\prime}}, y\right) \subseteq R\right\}
\end{aligned}
$$




\section{$\S 3$ The quantum group $U_{q}^{M}(\hat{\mathfrak{g}})$}

3.1 Quantum double. Let $H_{-}, H_{+}$be two arbitrary Hopf algebras on a ground field (or ring) $F$, and let $\pi:\left(H_{-}\right)_{o p} \otimes H_{+} \rightarrow F$ be any arbitrary Hopf pairing. The quantum double $D=D\left(H_{-}, H_{+}, \pi\right)$ is the algebra $T\left(H_{-} \oplus H_{+}\right) / \mathcal{R}$, where $T\left(H_{-} \oplus\right.$ $\left.H_{+}\right)$is the tensor algebra on $H_{-} \oplus H_{+}$and $\mathcal{R}$ is the ideal of relations

$$
\begin{aligned}
& 1_{H_{-}}=1=1_{H_{+}}, \quad x \otimes y=x y \quad \text { for } x, y \in H_{+} \text {or } x, y \in H_{-} \\
& \sum_{(x),(y)} \pi\left(y_{(2)}, x_{(2)}\right) x_{(1)} \otimes y_{(1)}=\sum_{(x),(y)} \pi\left(y_{(1)}, x_{(1)}\right) y_{(2)} \otimes x_{(2)} \quad \text { for } x \in H_{+}, y \in H_{-} \text {. }
\end{aligned}
$$

Then (cf. [CP], $\S 4$ 4.2.D) $D$ has a canonical structure of Hopf algebra such that $H_{-}$, $H_{+}$are Hopf subalgebras of it and multiplication yields isomorphisms of coalgebras

$$
H_{+} \otimes H_{-} \longleftrightarrow D \otimes D \stackrel{m}{\longrightarrow} D, \quad H_{-} \otimes H_{+} \longleftrightarrow D \otimes D \stackrel{m}{\longrightarrow} D .
$$

Now take $D_{q}^{M}(\hat{\mathfrak{g}}):=D\left(U_{\leq}^{Q_{\infty}}, U_{\geq}^{M}, \pi\right)$ : by definition, $D_{q}^{M}(\hat{\mathfrak{g}})$ is generated by $K_{\alpha}, L_{\mu}$, $F_{i}, E_{i}$ - identified with $1 \otimes K_{\alpha}, L_{\mu} \otimes 1,1 \otimes F_{i}, E_{i} \otimes 1$ when thinking at $D_{q}^{M}(\hat{\mathfrak{g}}) \cong$ $U_{\geq}^{M} \otimes U_{\leq}^{Q_{\infty}}-\left(\alpha \in Q_{\infty}, \mu \in M, i \in I\right)$, while the relations defining $\mathcal{R}$ reduce to

$$
\begin{gathered}
K_{\alpha} L_{\mu}=L_{\mu} K_{\alpha}, \quad K_{\alpha} E_{j}=q^{+\left(\alpha_{j} \mid \alpha\right)} E_{j} K_{\alpha}, \quad L_{\mu} F_{j}=q^{-\left(\alpha_{j} \mid \mu\right)} F_{j} L_{\mu} \\
E_{i} F_{j}-F_{j} E_{i}=\delta_{i j} \frac{L_{\alpha_{i}}-K_{-\alpha_{i}}}{q_{i}-q_{i}^{-1}}
\end{gathered}
$$

Finally, PBW bases of quantum Borel algebras provide PBW bases of $D_{q}^{M}(\hat{\mathfrak{g}})$. In the sequel we shall also use the notation $D_{M}:=D_{q}^{M}(\hat{\mathfrak{g}})$.

3.2 The quantum algebra $U_{q}^{M}(\hat{\mathfrak{g}})$. Let $\mathfrak{K}^{M}$ be the ideal of $D_{q}^{M}(\hat{\mathfrak{g}})$ generated by the elements $L \otimes 1-1 \otimes L, L \in U_{0}^{M}$; then $\mathfrak{K}^{M}$ is in fact a Hopf ideal, whence $D_{q}^{M}(\hat{\mathfrak{g}}) / \mathfrak{K}^{M}$ is a Hopf algebra. The presentation above yields one of $U_{q}^{M}(\hat{\mathfrak{g}}):=D_{q}^{M}(\hat{\mathfrak{g}}) / \mathfrak{K}^{M}:$ it is the associative $\mathbb{C}(q)$-algebra with 1 given by generators $F_{i}, L_{\mu}, E_{i}$ and relations

$$
\begin{gathered}
L_{0}=1, \quad L_{\mu} L_{\nu}=L_{\mu+\nu}=L_{\nu} L_{\mu}, \quad E_{i} F_{h}-F_{h} E_{i}=\delta_{i h} \frac{L_{\alpha_{i}}-L_{-\alpha_{i}}}{q_{i}-q_{i}^{-1}} \\
L_{\mu} F_{i}=q^{-\left(\alpha_{i} \mid \mu\right)} F_{i} L_{\mu}, \quad \sum_{k=0}^{1-a_{i j}}(-1)^{k}\left[\begin{array}{c}
1-a_{i j} \\
k
\end{array}\right]_{q_{i}} F_{i}^{1-a_{i j}-k} F_{j} F_{i}^{k}=0 \\
L_{\mu} E_{i}=q^{+\left(\alpha_{i} \mid \mu\right)} E_{i} L_{\mu}, \quad \sum_{k=0}^{1-a_{i j}}(-1)^{k}\left[\begin{array}{c}
1-a_{i j} \\
k
\end{array}\right]_{q_{i}} E_{i}^{1-a_{i j}-k} E_{j} E_{i}^{k}=0
\end{gathered}
$$

(for all $\mu \in M, i, j, h \in I$ with $i \neq j$ ) with the Hopf structure given by

$$
\begin{array}{clc}
\Delta\left(F_{i}\right)=F_{i} \otimes L_{-\alpha_{i}}+1 \otimes F_{i}, & \epsilon\left(F_{i}\right)=0, & S\left(F_{i}\right)=-F_{i} L_{\alpha_{i}} \\
\Delta\left(L_{\mu}\right)=L_{\mu} \otimes L_{\mu}, & \epsilon\left(L_{\mu}\right)=1, & S\left(L_{\mu}\right)=L_{-\mu} \\
\Delta\left(E_{i}\right)=E_{i} \otimes 1+L_{\alpha_{i}} \otimes F_{i}, & \epsilon\left(E_{i}\right)=0, & S\left(F_{i}\right)=-L_{-\alpha_{i}} E_{i}
\end{array}
$$


Finally, let $\operatorname{pr}_{M}: D_{q}^{M}(\hat{\mathfrak{g}}) \longrightarrow D_{q}^{M}(\hat{\mathfrak{g}}) / \mathfrak{K}^{M}=: U_{q}^{M}(\hat{\mathfrak{g}})$ be the canonical Hopf algebra epimorphism; we shall also use notation $K_{\alpha}:=L_{\alpha}, M_{\mu}:=L_{\mu}$, for all $\alpha \in Q_{\infty}, \mu \in M$.

3.3 Integer forms of $U_{q}^{M}(\hat{\mathfrak{g}})$. Let $\mathfrak{U}^{M}(\hat{\mathfrak{g}})$ be the $R$-subalgebra of $U_{q}^{M}(\hat{\mathfrak{g}})$ generated by $\left\{F_{i}^{(\ell)},\left(\begin{array}{c}M_{j} ; c \\ t\end{array}\right), M_{j}^{-1}, E_{i}^{(m)} \mid \ell, c, t, m \in \mathbb{N} ; i \in I, j \in I_{\infty}\right\}$; this is a Hopf subalgebra of $U_{q}^{M}(\hat{\mathfrak{g}})$ (cf. [Lu1], [DL]), with PBW basis over $R$ (cf. [Ga2])

$$
\left\{\prod_{\alpha \in \widetilde{\Phi}_{+}} E_{\alpha}^{\left(n_{\alpha}\right)} \cdot \prod_{j \in I_{\infty}}\left(\begin{array}{c}
M_{j} ; 0 \\
t_{j}
\end{array}\right) M_{j}^{-E n t\left(t_{j} / 2\right)} \cdot \prod_{\alpha \in \widetilde{\Phi}_{+}} F_{\alpha}^{\left(m_{\alpha}\right)} \mid n_{\alpha}, t_{j}, m_{\alpha} \in \mathbb{N}, \forall \alpha, j\right\} ;
$$

this is also a $\mathbb{C}(q)$-basis of $U_{q}^{M}(\hat{\mathfrak{g}})$, hence $\mathfrak{U}^{M}(\hat{\mathfrak{g}})$ is an $R$-form of $U_{q}^{M}(\hat{\mathfrak{g}})$.

Let $\mathcal{U}^{M}(\hat{\mathfrak{g}})$ be the $R$-subalgebra of $U_{q}^{M}(\hat{\mathfrak{g}})$ generated by $\left\{\bar{F}_{\alpha} \mid \alpha \in \widetilde{\Phi}_{+}\right\} \cup\left\{M_{j}^{ \pm 1} \mid j \in\right.$ $\left.I_{\infty}\right\} \cup\left\{\bar{E}_{\alpha} \mid \alpha \in \widetilde{\Phi}_{+}\right\}$(cf. [BK], §3); this also is a Hopf subalgebra, with PBW basis

$$
\left\{\prod_{\alpha \in \widetilde{\Phi}_{+}} \bar{E}_{\alpha}^{n_{\alpha}} \cdot \prod_{j \in I_{\infty}} M_{j}^{t_{j}} \cdot \prod_{\alpha \in \widetilde{\Phi}_{+}} \bar{F}_{\alpha}^{m_{\alpha}} \mid t_{i} \in \mathbb{Z}, n_{\alpha}, m_{\alpha} \in \mathbb{N}, \forall j, \alpha\right\}
$$

(over $R$ ); the latter is also a $\mathbb{C}(q)$-basis of $U_{q}^{M}(\hat{\mathfrak{g}})$, hence $\mathcal{U}^{M}(\hat{\mathfrak{g}})$ is an $R$-form of $U_{q}^{M}(\hat{\mathfrak{g}})$.

Like for quantum Borel algebras, the same forms can also be defined using modified root vectors, hence they have also PBW bases of ordered monomials in the $M_{i}$ 's and the modified root vectors.

3.4 Specialization at roots of 1 and quantum Frobenius morphisms. In sight of specializations, $\mathbb{C}$ will be thought of as an $R$-algebra via $\mathbb{C} \cong R /(q-c)$, for all $c \in \mathbb{C} \backslash\{0\}$. Let $\varepsilon$ be a primitive $\ell$-th root of 1 , for $\ell$ odd, $\ell>d:=\max _{i}\left\{d_{i}\right\}_{i}$, or $\ell=1$. Then we set $\mathfrak{U}_{\varepsilon}^{M}(\hat{\mathfrak{g}}):=\mathfrak{U}^{M}(\hat{\mathfrak{g}}) /(q-\varepsilon) \mathfrak{U}^{M}(\hat{\mathfrak{g}}) \cong \mathfrak{U}^{M}(\hat{\mathfrak{g}}) \otimes_{R} \mathbb{C}$. When $\ell=1$ (i. e. $\varepsilon=1$ ) it is well-known (cf. e. g. [DL]) $)^{2}$ that $\mathfrak{U}_{1}^{M}(\hat{\mathfrak{g}})$ is a Poisson Hopf coalgebra, and we have a Poisson Hopf coalgebra isomorphism

$$
\mathfrak{U}_{1}^{M}(\hat{\mathfrak{g}}) \cong U(\hat{\mathfrak{g}})
$$

in a word, $\mathfrak{U}^{M}(\hat{\mathfrak{g}})$ specializes to $U(\hat{\mathfrak{g}})$ for $q \rightarrow 1$ : in symbols, $\mathfrak{U}^{M}(\hat{\mathfrak{g}}) \stackrel{q \rightarrow 1}{\longrightarrow} U(\hat{\mathfrak{g}})$.

Remark: this point deserves some care. Following Lusztig (cf. for instance [Lu2]), people usually specialize $U_{q}(\hat{\mathfrak{g}})$ at $q=1$ by taking an integer form which is slightly different from our $\mathfrak{U}^{M}(\hat{\mathfrak{g}})$ : to be precise, it is defined like $\mathfrak{U}^{Q}(\hat{\mathfrak{g}})$ but with $\left[\begin{array}{c}K_{i} ; c \\ t\end{array}\right]:=$ $\prod_{s=1}^{t} \frac{q_{i}^{c-s+1} K_{i}-q_{i}^{-c+s-1} K_{i}^{-1}}{q_{i}^{s}-q_{i}^{-s}}$ instead of $\left(\begin{array}{c}K_{i} ; c \\ t\end{array}\right):=\prod_{s=1}^{t} \frac{q_{i}^{c-s+1} K_{i}-1}{q_{i}^{s}-1}$ (hence its toral part

${ }^{2}$ [loc. cit.] deals with the finite type case and quantum groups of adjoint type, but its arguments apply to the present situation too. 
is smaller). In particular one has $K_{i}-K_{i}^{-1}=\left(q_{i}-q_{i}^{-1}\right) \cdot\left[\begin{array}{c}K_{i} ; 0 \\ 1\end{array}\right]$, which at $q=1$ gives $K_{i}^{2}=1$ : thus for $q \rightarrow 1$ what one gets is only a central extension of $U(\hat{\mathfrak{g}})$, namely $U(\hat{\mathfrak{g}}) \otimes_{\mathbb{C}} \mathbb{C}\left[K_{0}, K_{1}, \ldots, K_{n}\right]$, with the $K_{i}$ 's central, group-like, and idempotents.

On the other hand, in our $\mathfrak{U}^{Q}(\hat{\mathfrak{g}})$ (and similarly for $\mathfrak{U}^{M}(\hat{\mathfrak{g}})$, in general) we have $K_{i}-1=\left(q_{i}-1\right) \cdot\left(\begin{array}{c}K_{i} ; 0 \\ 1\end{array}\right)$, which at $q=1$ gives $K_{i}=1$, so that (3.4) holds.

When $\ell>1$ we have an epimorphism (cf. [Lu1], [DL])

$$
\mathfrak{F r}_{\hat{\mathfrak{g}}}: \mathfrak{U}_{\varepsilon}^{M}(\hat{\mathfrak{g}}) \longrightarrow \mathfrak{U}_{1}^{M}(\hat{\mathfrak{g}}) \cong U(\hat{\mathfrak{g}})
$$

of Hopf algebras defined by (for all $i \in I, j \in I_{\infty}$, with $M_{j}:=L_{\mu_{j}}$ )

$$
\mathfrak{F}_{\mathfrak{\mathfrak { g }}}:\left\{\begin{array}{l}
\left.\left.F_{i}^{(s)}\right|_{q=\varepsilon} \mapsto F_{i}^{(s / \ell)}\right|_{q=1},\left.\left.\left(\begin{array}{c}
M_{j} ; 0 \\
s
\end{array}\right)\right|_{q=\varepsilon} \mapsto\left(\begin{array}{c}
M_{j} ; 0 \\
s / \ell
\end{array}\right)\right|_{q=1},\left.\left.E_{i}^{(s)}\right|_{q=\varepsilon} \mapsto E_{i}^{(s / \ell)}\right|_{q=1} \text { if } \ell \mid s \\
\left.F_{i}^{(s)}\right|_{q=\varepsilon} \mapsto 0,\left.\quad\left(\begin{array}{c}
M_{j} ; 0 \\
s
\end{array}\right)\right|_{q=\varepsilon} \mapsto 0,\left.\quad E_{i}^{(s)}\right|_{q=\varepsilon} \mapsto 0 \text { otherwise } \\
\left.M_{i}^{-1}\right|_{q=1} \mapsto 1
\end{array}\right.
$$

As it is usual in literature, we call $\mathfrak{F}_{\mathfrak{\mathfrak { g }}}$ a quantum Frobenius morphism (cf. [Lu1]). Similarly, we set $\mathcal{U}_{\varepsilon}^{M}(\hat{\mathfrak{g}}):=\mathcal{U}^{M}(\hat{\mathfrak{g}}) /(q-\varepsilon) \mathcal{U}^{M}(\hat{\mathfrak{g}}) \cong \mathcal{U}^{M}(\hat{\mathfrak{g}}) \otimes_{R} \mathbb{C}$; when $\ell=1$ it is

$$
\mathcal{U}_{1}^{M}(\hat{\mathfrak{g}}) \cong F\left[\widehat{H}_{M}\right]
$$

as Poisson Hopf algebras over $\mathbb{C}$ (see $[\mathrm{BK}], \S 5)$ : here $\widehat{H}_{M}$ is a Poisson proalgebraic group with tangent Lie bialgebra $\hat{\mathfrak{h}}$ : it is simply an extension - by adding two copies of $\mathbb{C}^{*}$ to the maximal torus - of the Poisson proalgebraic group $\Omega$ which is defined in [BK], $\S 4$. In a word, $\mathcal{U}^{M}(\hat{\mathfrak{g}})$ specializes to $F\left[\widehat{H}_{M}\right]$ as $q \rightarrow 1$, or $\mathcal{U}^{M}(\hat{\mathfrak{g}}) \stackrel{q \rightarrow 1}{\longrightarrow} F\left[\widehat{H}_{M}\right]$.

When $\ell>1$, assume in addition that the following technical condition is satisfied:

$$
\begin{array}{cc}
\text { g.c.d. }(\ell, n+1)=1 & \text { if } \mathfrak{g} \text { is of type } A_{n} \text { or } C_{n} \\
\text { g.c.d. }(\ell, 2 n-1)=1 & \text { if } \mathfrak{g} \text { is of type } B_{n} \\
\text { g.c.d. }(\ell, n-1)=1 & \text { if } \mathfrak{g} \text { is of type } D_{n} \\
\text { g.c.d. }(\ell, 3)=1 & \text { if } \mathfrak{g} \text { is of type } E_{6} \text { or } G_{2}
\end{array}
$$

Then from $[\mathrm{BK}], \S 3$, we record the existence of a Hopf algebra monomorphism

$$
\mathcal{F} r_{\hat{\mathfrak{g}}}: F\left[\widehat{H}_{M}\right] \cong \mathcal{U}_{1}^{M}(\hat{\mathfrak{g}}) \longleftrightarrow \mathcal{U}_{\varepsilon}^{M}(\hat{\mathfrak{g}})
$$

which we call again quantum Frobenius morphism; it is uniquely determined by

$$
\mathcal{F} r_{\hat{\mathfrak{g}}}:\left.\left.\quad \bar{F}_{\alpha}\right|_{q=1} \mapsto \bar{F}_{\alpha}^{\ell}\right|_{q=\varepsilon},\left.\left.\quad L_{\mu}\right|_{q=1} \mapsto L_{\mu}^{\ell}\right|_{q=\varepsilon},\left.\left.\bar{E}_{\alpha}\right|_{q=1} \mapsto \bar{E}_{\alpha}^{\ell}\right|_{q=\varepsilon}
$$


for all $\alpha \in \widetilde{\Phi}_{+}, \mu \in M$ (this is a renormalized version of that in [BK]), and it enjoys

$\mathcal{F} r_{\hat{\mathfrak{g}}}:\left.\left.\bar{F}_{(r \delta, i)}\right|_{q=1} \mapsto \ell \bar{F}_{(r \ell \delta, i)}\right|_{q=\varepsilon},\left.\left.\bar{E}_{(r \delta, i)}\right|_{q=1} \mapsto \ell \bar{E}_{(r \ell \delta, i)}\right|_{q=\varepsilon} \quad \forall(r \delta, i) \in \widetilde{\Phi}_{+}^{\mathrm{im}} ;$

finally the image of $\mathcal{F} r_{\hat{\mathfrak{g}}}$ is the center of $\mathcal{U}_{\varepsilon}^{M}(\hat{\mathfrak{g}})$, i.e.

$$
\mathcal{F} r_{\hat{\mathfrak{g}}}\left(\mathcal{U}_{1}^{M}(\hat{\mathfrak{g}})\right)=Z\left(\mathcal{U}_{\varepsilon}^{M}(\hat{\mathfrak{g}})\right)=: Z_{\varepsilon}
$$

\section{$\S 4$ Quantum formal groups}

4.1 Formal Hopf algebras and quantum formal groups. In this subsection we introduce the notion of quantum formal group. Recall (cf. [Di], ch. I) that formal groups can be defined in a category of a special type of commutative topological algebras, whose underlying vector space (or module) is linearly compact; following Drinfel'd's philosophy,we define quantum formal groups by simply dropping out any commutativity assumption of the classical notion of formal group; thus now we quickly outline how to modify the latter (following [Di], ch. I) in order to define our new quantum objects.

Let $E$ be any vector space over a field $K$ (one can then generalize more or less wathever follows to the case of free modules over a ring), and let $E^{*}$ be its (linear) dual; we write $\left\langle x^{*}, x\right\rangle$ for $x^{*}(x)$ for $x \in E, x^{*} \in E^{*}$. We consider on $E^{*}$ the weak $*$-topology, i. e. the coarsest topology such that for each $x \in E$ the linear map $x^{*} \mapsto\left\langle x^{*}, x\right\rangle$ of $E^{*}$ into $K$ is continuous, when $K$ is given the discrete topology. We can describe this topology by choosing a basis $\left\{e_{i}\right\}_{i \in I}$ of $E$ : to each $i \in I$ we associate the linear (coordinate) form $e_{i}^{*}$ on $E$ such that $\left\langle e_{i}^{*}, e_{j}\right\rangle=\delta_{i j}$, and we say that the family $\left\{e_{i}^{*}\right\}_{i \in I}$ is the pseudobasis of $E^{*}$ dual to $\left\{e_{i}\right\}_{i \in I}$; then the subspace $E^{\prime}$ of $E$ which is (algebraically) generated by the $e_{i}^{*}$ is dense in $E^{*}$, and $E^{*}$ is nothing but the completion of $E^{\prime}$, when $E^{\prime}$ is given the topology for which a fundamental system of neighborhoods of 0 consists of the vector subspaces containing almost all the $e_{i}^{*}$; thus elements of $E^{*}$ can be described by series in the $e_{i}^{*}$ 's which in the given topology are in fact convergent. Finally, the topological vector spaces $E^{*}$ are characterized by the property of linear compactness.

Let now $E, F$ be any two vector spaces over $K$, and $u: E \rightarrow F$ a linear map; then the dual map $u^{*}: F^{*} \rightarrow E^{*}$ is continuous, and conversely for any linear map $v: F^{*} \rightarrow E^{*}$ which is continuous there exists a unique linear map $u: E \rightarrow F$ such that $v=u^{*}$.

The tensor product $E^{*} \otimes F^{*}$ is naturally identified to a subspace of $(E \otimes F)^{*}$ by $\left\langle x^{*} \otimes y^{*}, x \otimes y\right\rangle=\left\langle x^{*}, x\right\rangle \cdot\left\langle y^{*}, y\right\rangle$; thus if $\left\{e_{i}\right\}_{i \in I}$ and $\left\{f_{j}\right\}_{j \in J}$ are bases of $E$ and $F$, and $\left\{e_{i}^{*}\right\}_{i \in I}$ and $\left\{f_{j}^{*}\right\}_{j \in J}$ their dual pseudobases in $E^{*}$ and $F^{*}$, then $\left\{e_{i}^{*} \otimes f_{j}^{*}\right\}_{i \in I, j \in J}$ is the dual pseudobasis of $\left\{e_{i} \otimes f_{j}\right\}_{i \in I, j \in J}$ in $(E \otimes F)^{*}$. Thus $(E \otimes F)^{*}$ is the completion of $E^{*} \otimes F^{*}$ for the tensor product topology, i. e. the topology of $E^{*} \otimes F^{*}$ for which 
a fundamental system of neighborhoods of 0 consists of the sets $E^{*} \otimes V+W \otimes F^{*}$ where $V$, resp. $W$, ranges in a fundamental system of neighborhoods of 0 made of vector subspaces; we denote this completion by $E^{*} \widehat{\otimes} F^{*}$, and we call it the completed (or topological) tensor product of $E^{*}$ and $F^{*}$; the embedding $E^{*} \otimes F^{*} \hookrightarrow(E \otimes F)^{*}=$ $E^{*} \widehat{\otimes} F^{*}$ is then continuous. Finally, if $u: E_{1} \rightarrow E_{2}, v: F_{1} \rightarrow F_{2}$ are linear maps, then $(u \otimes v)^{*}:\left(E_{2} \otimes F_{2}\right)^{*}=E_{2}{ }^{*} \widehat{\otimes} F_{2}{ }^{*} \longrightarrow\left(E_{1} \otimes F_{1}\right)^{*}=E_{1}{ }^{*} \widehat{\otimes} F_{1}{ }^{*}$ coincides with the continuous extension to $E_{2}{ }^{*} \widehat{\otimes} F_{2}{ }^{*}$ of the continuous map $u^{*} \otimes v^{*}: E_{2}{ }^{*} \otimes F_{2}{ }^{*} \rightarrow$ $E_{1}{ }^{*} \otimes F_{1}{ }^{*}$; thus it is also denoted by $u^{*} \widehat{\otimes} v^{*}$.

We define a linearly compact algebra to be a topological algebra whose underlying vector space (or free module) is linearly compact: then linearly compact algebras form a full subcategory of the category of topological algebras; morever, for any two objects $A_{1}$ and $A_{2}$ in this category, their topological tensor product $A_{1} \widehat{\otimes} A_{2}$ is defined. Dually, within the category of linearly compact vector spaces we define linearly compact coalgebras as triplets $(C, \Delta, \epsilon)$ with $\Delta: C \rightarrow C \widehat{\otimes} C$ and $\epsilon: C \rightarrow K$ satisfying the usual coalgebra axioms. The arguments in [Di] (which never require commutativity nor cocommutativity) show that ()$^{*}:(A, m, 1) \mapsto\left(A^{*}, m^{*}, 1^{*}\right)$ defines a contravariant functor from algebras to linearly compact coalgebras, while ()$^{*}:(C, \Delta, \epsilon) \mapsto\left(C^{*}, \Delta^{*}, \epsilon^{*}\right)$ defines a contravariant functor from coalgebras to linearly compact algebras. Finally, we define a formal Hopf algebra as a datum $(H, m, 1, \Delta, \epsilon, S)$ such that $(H, m, 1)$ is a linearly compact algebra, $(H, \Delta, \epsilon)$ is a linearly compact coalgebra, and the usual compatibility axioms of Hopf algebras are satisfied. "Usual" Hopf algebras are particular cases of formal Hopf algebras.

We define quantum formal group the spectrum of a formal Hopf algebra (whereas classical formal groups are spectra of commutative formal Hopf algebras: cf. [Di], ch. I).

Our goal is to study $U_{q}^{M}(\hat{\mathfrak{g}})^{*}$. Since $U_{q}^{M}(\hat{\mathfrak{g}})$ is a Hopf algebra, its linear dual $U_{q}^{M}(\hat{\mathfrak{g}})^{*}$ is a formal Hopf algebra. The functor ()$^{*}$ turns the natural epimorphism $p r_{M}: D_{M} \longrightarrow$ $U_{q}^{M}(\hat{\mathfrak{g}})$ into a monomorphism $j_{M^{\prime}}:=\left(p r_{M}\right)^{*}: U_{q}^{M}(\hat{\mathfrak{g}})^{*} \longleftrightarrow D_{M}{ }^{*}$ of formal Hopf algebras: therefore we begin by studying $D_{M}{ }^{*}$. The following is straightforward:

Proposition 4.2. Let $H_{-}, H_{+}$be Hopf F-algebras, let $\pi:\left(H_{-}\right)_{o p} \otimes H_{+} \longrightarrow F$ be an arbitrary Hopf pairing, and let $D:=D\left(H_{-}, H_{+}, \pi\right)$ be the corresponding quantum double. Then there exist $F$-algebra isomorphisms

$$
D^{*} \cong H_{+}{ }^{*} \widehat{\otimes} H_{-}{ }^{*}, \quad D^{*} \cong H_{-}{ }^{*} \widehat{\otimes} H_{+}{ }^{*}
$$

dual of the $F$-coalgebra isomorphisms $D \cong H_{+} \otimes H_{-}, D \cong H_{-} \otimes H_{+}$(cf. §3.1).

4.3 Quantum enveloping algebras as function algebras. The DRT pairings induce several linear embeddings, namely

$$
\begin{aligned}
& U_{-} \longleftrightarrow U_{+}^{*}, \quad i m_{M}: U_{0}^{M} \longleftrightarrow U_{0}^{M^{\prime}}{ }^{*}, \quad U_{\leq}^{M} \longleftrightarrow U_{\geq}^{M^{\prime}} \quad \text { (induced by } \pi \text { ) } \\
& U_{+} \hookrightarrow U_{-}^{*}, \quad \overline{i m}_{M}: U_{0}^{M} \longleftrightarrow U_{0}^{M^{\prime}}{ }^{*}, \quad U_{\geq}^{M} \hookrightarrow U_{\leq}^{M^{\prime}} \text { * (induced by } \bar{\pi} \text { ) }
\end{aligned}
$$

the right-hand-side ones being also embeddings of formal Hopf algebras; thus we identify the various quantum algebras with their images in the corresponding dual spaces. 


\section{Lemma 4.4.}

(a) $\mathcal{U}_{-}$contains the pseudobasis of $U_{+}{ }^{*}$ dual of the PBW basis of $\mathfrak{U}_{+}$of decreasing ordered monomials, and $\mathfrak{U}_{-}$contains the pseudobasis of $U_{+}{ }^{*}$ dual of the PBW basis of $\mathcal{U}_{+}$of decreasing ordered monomials. Moreover, the PBW bases of $\mathcal{U}_{-}$and of $\mathfrak{U}_{-}$ are also pseudobases of $U_{+}{ }^{*}$. A similar statement holds with the roles of $U_{-}$and $U_{+}$ reversed.

(b) $\mathcal{U}_{0}^{M}$ (hence $U_{0}^{M}$ ) contains the pseudobasis $\mathcal{B}_{M}$ (relative to $i_{M}$ ), resp. $\overline{\mathcal{B}}_{M}$ (relative to $\overline{\mathrm{im}}_{M}$ ), of $U_{0}^{M^{\prime}}$, dual of the PBW basis of $\mathfrak{U}_{0}^{M^{\prime}}$.

(c) $\mathcal{U}_{\leq}^{M}$, resp. $\mathcal{U}_{\geq}^{M}$ (hence $U_{\leq}^{M}$, resp. $U_{\geq}^{M}$ ) contains the pseudobasis of $U_{\geq}^{M^{\prime}}$, resp. of $U_{\geq}^{M^{\prime *}}$, dual of the $P B W$ basis of $\mathfrak{U}_{\leq}^{M^{\prime}}$, resp. of $\mathfrak{U}_{\geq}^{M^{\prime}}$. Moreover, the PBW basis of $\mathcal{U}_{\leq}^{M}$, resp. $\mathcal{U}_{\geq}^{M}$, is itself a pseudobasis of $U_{\geq}^{M^{\prime *}}$, resp. of $U_{\geq}^{M^{\prime *}}$.

Proof. Part (a) of the statement follows from Lemma 2.5 and the Claim in $§ 2.7$.

As for $(b)$, let $u_{\tau}:=\prod_{i \in \infty}\left(\begin{array}{c}\Lambda_{i} ; 0 \\ t_{i}\end{array}\right) \cdot \Lambda_{i}^{-E n t\left(t_{i} / 2\right)}\left(\tau=\left(t_{0}, t_{1}, \ldots, t_{n}, t_{\infty}\right) \in \mathbb{N}^{n+2}\right)$ be any monomial in the PBW basis of $\mathfrak{U}_{0}^{M^{\prime}}$. Then direct computation gives

$$
\left\langle L_{-\mu}, u_{\tau}\right\rangle_{\pi}=\prod_{i \in \infty}\left(\begin{array}{c}
m_{i} \\
t_{i}
\end{array}\right)_{q_{i}} \cdot q^{-d_{i} m_{i} \cdot \operatorname{Ent}\left(t_{i} / 2\right)} \quad \forall \mu, \tau \in \mathbb{N}^{n+2}
$$

where we identify $M_{+} \cong \mathbb{N}^{n+2}$ so that $M_{+} \ni \mu=m_{0} \mu_{0}+m_{1} \mu_{1}+\cdots+m_{n} \mu_{n}+$ $m_{\infty} \mu_{\infty} \cong\left(m_{0}, m_{1}, \ldots, m_{n}, m_{\infty}\right) \in \mathbb{N}^{n+2}$. Then endowing $\mathbb{N}^{n+2}$ with the product ordering (of the natural ordering of $\mathbb{N}$ ) we have

$$
\begin{array}{ll}
\left\langle L_{-\mu}, u_{\tau}\right\rangle_{\pi} \neq 0 \Longleftrightarrow \tau \preceq \mu & \\
\left\langle L_{-\tau}, u_{\tau}\right\rangle_{\pi}=q^{-T(\tau)} & \forall \tau \in \mathbb{N}^{n}
\end{array}
$$

where $T(\tau):=\sum_{i \in I_{\infty}} d_{i} t_{i} \operatorname{Ent}\left(t_{i} / 2\right)$; in particular $q^{-T(\tau)}$ is invertible in $R$. Thus we have formulas (for all $\tau \in \mathbb{N}^{n+2}$ )

$$
L_{-\tau}=q^{-T(\tau)} \cdot u_{\tau}^{*}+\sum_{\tau^{\prime} \prec \tau}\left\langle L_{-\tau}, u_{\tau^{\prime}}\right\rangle_{\pi} \cdot u_{\tau^{\prime}}^{*}
$$

which tell us that $\left\{L_{-\tau} \mid \tau \in \mathbb{N}^{n+2}\right\}$ is obtained from $\left\{u_{\tau}^{*} \mid \tau \in \mathbb{N}^{n+2}\right\}$ by means of the matrix $\mathbb{M}:=\left(\left\langle L_{-\tau}, u_{\tau^{\prime}}\right\rangle_{\pi}\right)_{\tau, \tau^{\prime} \in \mathbb{N}^{n+2}}$ which has lower triangular shape, all entries in $R$, and diagonal entries invertible in $R$; then the inverse matrix $\mathbb{M}^{-1}$ has the same properties, whence (b) follows.

Finally, for $(c)$ note that $\pi(y \cdot \ell, x \cdot m)=\pi(y, x) \cdot \pi(\ell, m)$ for all $y \in U_{-}, \ell \in U_{0}^{M}$, $m \in U_{0}^{M^{\prime}}, x \in U_{+}$, as one sees at once from Lemma 2.5 (and similarly for $\bar{\pi}$ ); therefore, since the PBW basis of, say, $\mathfrak{U}_{\leq}^{M^{\prime}}$ is the tensor product of the like bases of $\mathfrak{U}_{+}$and $\mathfrak{U}_{0}^{M^{\prime}}$ then the dual pseudobasis of $\left(\mathfrak{U}_{\leq}^{M^{\prime}}\right)^{*}$ is the tensor product of the pseudobases of $\mathfrak{U}_{+}{ }^{*}$ and $\left(\mathfrak{U}_{0}^{M^{\prime}}\right)^{*}$ given in $(a)$ and $(b)$. With similar arguments all of $(c)$ is proved. 
4.5 Remark. Since $D_{M^{\prime}} \cong U_{\geq}^{M^{\prime}} \otimes U_{\leq}^{Q_{\infty}} \cong U_{+} \otimes U_{0}^{M^{\prime}} \otimes U_{0}^{Q_{\infty}} \otimes U_{-}$, we have $D_{M^{\prime}}{ }^{*} \cong U_{\geq}^{M^{\prime *}} \widehat{\otimes} U_{\leq}^{Q_{\infty}} \cong U_{+}{ }^{*} \widehat{\otimes} U_{0}^{M^{\prime *}} \widehat{\otimes} U_{0}^{Q_{\infty}} \widehat{\otimes} \widehat{\otimes} U_{-}{ }^{*} ;$ hence from Lemma 4.4 we deduce

Every element $f \in D_{M^{\prime}}{ }^{*}$ has a unique expression as formal series

$$
f=\sum_{\mathcal{F}, \mathcal{M}, \mathcal{L}, \mathcal{E}} a_{\mathcal{F}, \mathcal{M}, \mathcal{L}, \mathcal{E}} \cdot \mathcal{F} \cdot \mathcal{M} \cdot \mathcal{L} \cdot \mathcal{E}
$$

in which $a_{\mathcal{F}, \mathcal{M}, \mathcal{L}, \mathcal{E}} \in \mathbb{C}(q), \mathcal{M} \in \mathcal{B}_{M}, \mathcal{L} \in \overline{\mathcal{B}}_{P_{\infty}}$, and the $\mathcal{F}$ 's, resp. the $\mathcal{E}$ 's, are ordered monomials in the $F_{\alpha}$ 's, resp. in the $E_{\alpha}$ 's; then the natural evaluation pairing $D_{M^{\prime}}{ }^{*} \otimes D_{M^{\prime}} \rightarrow \mathbb{C}(q)$ is given by $\pi \otimes \pi \otimes \bar{\pi} \otimes \bar{\pi}$.

In particular, every $f \in D_{M^{\prime}}{ }^{*}$ can be uniquely expressed as a formal series in the $F_{\alpha}$ 's and the $E_{\alpha}$ 's $\left(\alpha \in \widetilde{\Phi}_{+}\right)$with coefficients in $\left(U_{0}^{M^{\prime}} \otimes U_{0}^{Q_{\infty}}\right)^{*} \cong U_{0}^{M^{\prime *}} \widehat{\otimes} U_{0}^{Q_{\infty} *}$.

Similarly the triangular decompositions $U_{+} \otimes U_{0}^{M^{\prime}} \otimes U_{-} \cong U_{q}^{M^{\prime}}(\hat{\mathfrak{g}}) \cong U_{-} \otimes U_{0}^{M^{\prime}} \otimes U_{+}$ give $U_{+}^{*} \widehat{\otimes} U_{0}^{M^{\prime}} \widehat{\otimes} U_{-}^{*} \cong U_{q}^{M^{\prime}}(\hat{\mathfrak{g}})^{*} \cong U_{-}^{*} \widehat{\otimes} U_{0}^{M^{\prime}}{ }^{*} \widehat{\otimes} U_{+}{ }^{*}$, whence Lemma 4.4 implies

Every $f \in U_{q}^{M^{\prime}}(\hat{\mathfrak{g}})^{*}$ can be uniquely expressed as a formal series in the $F_{\alpha}$ 's and the $E_{\alpha}$ 's $\left(\alpha \in \widetilde{\Phi}_{+}\right)$with coefficients in $U_{0}^{M^{\prime *}}$; then the natural evaluation pairing $U_{q}^{M^{\prime}}(\hat{\mathfrak{g}})^{*} \otimes U_{q}^{M^{\prime}}(\hat{\mathfrak{g}}) \rightarrow \mathbb{C}(q)$ can be described by $\pi \otimes \bar{\pi} \otimes \bar{\pi}$ or by $\pi \otimes \bar{\pi} \otimes \bar{\pi}$.

In particular, a pseudobasis of $U_{q}^{M^{\prime}}(\hat{\mathfrak{g}})^{*}$ can be given by taking the tensor product of pseudobases - for instance, those provided by Lemma $4.4-$ of $U_{+}{ }^{*},{U_{0}^{M^{\prime}}}^{*}$, and $U_{-}^{*}$; moreover, the (tensor) PBW basis of $\mathcal{U}_{-} \otimes \mathcal{U}_{0}^{M} \otimes \mathcal{U}_{+}$is a pseudobasis of $U_{q}^{M^{\prime}}(\hat{\mathfrak{g}})^{*}$.

In the sequel when considering the composed embedding $U_{0}^{M} \hookrightarrow U_{0}^{M^{\prime *}} \hookrightarrow U_{q}^{M^{\prime}}(\hat{\mathfrak{g}})^{*}$ we shall always mean that the first embedding is induced by $\bar{\pi}$ (cf. (4.1)); accordingly, the evaluation pairing $U_{q}^{M^{\prime}}(\hat{\mathfrak{g}})^{*} \otimes U_{q}^{M^{\prime}}(\hat{\mathfrak{g}}) \rightarrow \mathbb{C}(q)$ will be described by $\pi \otimes \bar{\pi} \otimes \bar{\pi}$, hence the pseudobasis of $U_{0}^{M^{\prime *}}$ to use is $\overline{\mathcal{B}}_{M}$.

Proposition 4.6. The monomorphism $j_{M}: U_{q}^{M^{\prime}}(\hat{\mathfrak{g}})^{*} \longleftrightarrow D_{M^{\prime}}{ }^{*}$ (cf. $\left.\S 4.1\right)$ is given by

$$
j_{M}: \quad F_{i} \mapsto F_{i} \otimes 1, \quad L_{\mu} \mapsto L_{-\mu} \otimes L_{\mu}, \quad E_{i} \mapsto 1 \otimes E_{i} \quad \forall i, \mu ;
$$

in particular the image of $j_{M}$ is the closure of the subalgebra generated by the set

$$
\left\{F_{i} \otimes 1, L_{-\mu} \otimes L_{\mu}, 1 \otimes E_{i} \mid i \in I_{\infty}, \mu \in M\right\} .
$$

Proof. For PBW monomials we have $\operatorname{pr}_{M}(E \cdot L \otimes K \cdot F)=E \cdot L \cdot K \cdot F$; therefore (4.2) comes out of the definition $j_{M}:=\left(p r_{M}\right)^{*}$. As an example

$$
\begin{gathered}
\left\langle j_{M}\left(L_{\mu}\right), E \cdot L_{\nu} \otimes K_{\alpha} \cdot F\right\rangle=\left\langle L_{\mu}, E \cdot L_{\nu} \cdot K_{\alpha} \cdot F\right\rangle=\delta_{E, 1} \cdot \delta_{F, 1} \cdot q^{(\mu \mid \nu+\alpha)} \\
\left\langle L_{-\mu} \otimes L_{\mu}, E \cdot L_{\nu} \otimes K_{\alpha} \cdot F\right\rangle_{\pi \otimes \bar{\pi}}=\delta_{E, 1} \cdot \delta_{F, 1} \cdot q^{(\mu \mid \nu+\alpha)}
\end{gathered}
$$

whence $j_{M}\left(L_{\mu}\right)=L_{-\mu} \otimes L_{\mu}$. Since $j_{M}:=\left(p r_{M^{\prime}}\right)^{*}$ is continuous (cf. $\left.\S 4.1\right)$, by Lemma 4.4 and Remark 4.5 it is uniquely determined by (4.2). 
Remark 4.7. Thanks to the previous results, we can identify $j_{M}\left(U_{q}^{M^{\prime}}(\hat{\mathfrak{g}})^{*}\right)$ with the space of formal series in the $F_{\alpha}$ 's and the $E_{\alpha}$ 's $\left(\alpha \in \widetilde{\Phi}_{+}\right)$with coefficients in $U_{0}^{M^{\prime}}$, using Proposition 4.6 and the similar identification for $U_{q}^{M^{\prime}}(\hat{\mathfrak{g}})^{*}$ (cf. Remark 4.5). Then similar remarks to those in Remark 4.5 hold.

4.8 Integer forms. We want to study the subspaces of linear functions on $U_{q}^{M^{\prime}}(\hat{\mathfrak{g}})$ which are "integer-valued" on its integer forms. Thus we define

$$
\begin{gathered}
\mathfrak{U}^{M^{\prime}}(\hat{\mathfrak{g}})^{*}:=\left\{f \in U_{q}^{M^{\prime}}(\hat{\mathfrak{g}})^{*} \mid\left\langle f, \mathfrak{U}^{M^{\prime}}(\hat{\mathfrak{g}})\right\rangle \subseteq R\right\}, \mathcal{U}^{M^{\prime}}(\hat{\mathfrak{g}})^{*}:=\left\{f \in U_{q}^{M^{\prime}}(\hat{\mathfrak{g}})^{*} \mid\left\langle f, \mathcal{U}^{M^{\prime}}(\hat{\mathfrak{g}})\right\rangle \subseteq R\right\} \\
\mathfrak{I}^{M}:=\left\{f \in j_{M}\left(U_{q}^{M^{\prime}}(\hat{\mathfrak{g}})^{*}\right) \mid\left\langle f, \mathfrak{U}^{M^{\prime}}(\hat{\mathfrak{g}})\right\rangle \subseteq R\right\}, \mathcal{I}^{M}:=\left\{f \in j_{M}\left(U_{q}^{M^{\prime}}(\hat{\mathfrak{g}})^{*}\right) \mid\left\langle f, \mathcal{U}^{M^{\prime}}(\hat{\mathfrak{g}})\right\rangle \subseteq R\right\} \\
\mathfrak{U}_{0}^{M^{\prime \prime}}:=\left\{f \in U_{0}^{M^{\prime}} \mid\left\langle f, \mathfrak{U}_{0}^{M^{\prime}}\right\rangle \subseteq R\right\}, \quad \mathcal{U}_{0}^{M^{\prime *}}:=\left\{f \in U_{0}^{M^{\prime *}} \mid\left\langle f, \mathcal{U}_{0}^{M^{\prime}}\right\rangle \subseteq R\right\} ;
\end{gathered}
$$

notice that $j_{M}$ restricts to isomorphisms $j_{M}: \mathfrak{U}^{M^{\prime}}(\hat{\mathfrak{g}})^{*} \stackrel{\cong}{\longrightarrow} \mathfrak{I}^{M}, j_{M}: \mathcal{U}^{M^{\prime}}(\hat{\mathfrak{g}})^{*} \stackrel{\cong}{\longrightarrow} \mathcal{I}^{M}$.

\section{Proposition 4.9.}

(a) $\mathfrak{U}^{M^{\prime}}(\hat{\mathfrak{g}})^{*}$ is the $R$-submodule (of $U_{q}^{M^{\prime}}(\hat{\mathfrak{g}})^{*}$ ) of all formal series (cf. $\S 4.5$ ) $\sum_{\mathcal{F}, \psi, \mathcal{E}} \mathcal{F} \cdot \psi \cdot \mathcal{E}$ in which $\psi \in \mathfrak{U}_{0}^{M^{\prime}}{ }^{*}$ and the $\mathcal{F}$ 's, resp. the $\mathcal{E}$ 's, are monomials of the $P B W$ basis of $\mathcal{U}_{-}$, resp. of $\mathcal{U}_{+}$. So $\mathfrak{U}^{M^{\prime}}(\hat{\mathfrak{g}})^{*}$ is a formal Hopf subalgebra of $U_{q}^{M^{\prime}}(\hat{\mathfrak{g}})^{*}$.

(b) $\mathcal{U}^{M^{\prime}}(\hat{\mathfrak{g}})^{*}$ is the $R$-submodule (of $U_{q}^{M^{\prime}}(\hat{\mathfrak{g}})^{*}$ ) of all formal series (cf. $\S 4.5$ ) $\sum_{\mathfrak{F}, \phi, \mathfrak{E}} \mathfrak{F} \cdot \phi \cdot \mathfrak{E}$ in which $\phi \in \mathcal{U}_{0}^{M^{\prime *}}$ and the $\mathfrak{F}$ 's, resp. the $\mathfrak{E}$ 's, are monomials of the PBW basis of $\mathfrak{U}_{-}$, resp. of $\mathfrak{U}_{+}$. So $\mathcal{U}^{M^{\prime}}(\hat{\mathfrak{g}})^{*}$ is a formal Hopf subalgebra of $U_{q}^{M^{\prime}}(\hat{\mathfrak{g}})^{*}$.

Proof. Let us prove (b), the proof for (a) being completely similar.

Let $f \in U_{q}^{M^{\prime}}(\hat{\mathfrak{g}})^{*}$ be given, and expand it as a series $f=\sum_{\phi, \eta} \mathfrak{F}_{\phi} \cdot \Phi_{\phi, \eta} \cdot \mathfrak{E}_{\eta}$ in which the $\mathfrak{F}_{\phi}$ 's, resp. the $\mathfrak{E}_{\eta}$ 's, are PBW monomials of $\mathfrak{U}_{-}$, resp. of $\mathfrak{U}_{+}$, and $\Phi_{\phi, \eta} \in U_{0}^{M^{\prime \prime}}$. Now fix $\bar{\eta}$, and let $\mathcal{E}_{\bar{\eta}}$ be the PBW-like monomial of $\mathcal{U}_{+}$which is (up to $\pm q^{s}$, for some $s \in \mathbb{Z}$ ) the dual of $\mathfrak{F}_{\bar{\eta}}$ : according to Lemma 2.5 , this is an ordered product of the $\bar{E}_{\gamma}$ 's $\left(\gamma \in \Phi_{+}^{\mathrm{re}}\right)$ and of the duals of the $\frac{r}{[r]_{q_{i}}} F_{(r \delta, i)}$ 's $\left((r \delta, i) \in \widetilde{\Phi}_{+}^{\mathrm{im}}\right)$; similarly, fix $\bar{\phi}$ and let $\mathcal{F}_{\bar{\phi}}$ be the PBW-like monomial of $\mathcal{U}_{-}$which is the dual (up to $\pm q^{t}$, for some $t \in \mathbb{Z}$ ) of $\mathfrak{E}_{\bar{\phi}}$ : thanks to the Claim in $\S 2.7$, we have $\mathcal{E}_{\bar{\eta}}, \mathcal{F}_{\bar{\phi}} \in \mathcal{U}^{M}(\hat{\mathfrak{g}})$. Fix also $\nu \in M^{\prime}$ : then

$$
\left\langle f, \mathcal{E}_{\bar{\eta}} \cdot L_{\nu} \cdot \mathcal{F}_{\bar{\phi}}\right\rangle=\sum_{\phi, \eta}\left\langle\mathfrak{F}_{\phi} \cdot \Phi_{\phi, \eta} \cdot \mathfrak{E}_{\eta}, \mathcal{E}_{\bar{\eta}} \cdot L_{\nu} \cdot \mathcal{F}_{\bar{\phi}}\right\rangle= \pm q^{s+t}\left\langle\Phi_{\bar{\phi}, \bar{\eta}}, L_{\nu}\right\rangle
$$

Therefore, since the monomials $\mathcal{E}_{\bar{\eta}} \cdot L_{\nu} \cdot \mathcal{F}_{\bar{\phi}}$ form a (PBW-like) basis of $\mathcal{U}^{M^{\prime}}(\hat{\mathfrak{g}})$, we have $f \in U_{q}^{M^{\prime}}(\hat{\mathfrak{g}})^{*}$ if and only if $\Phi_{\bar{\phi}, \bar{\eta}} \in \mathcal{U}_{0}^{M^{\prime *}}$, q.e.d.

Now consider the Hopf structure. Let $f \in \mathcal{U}^{M^{\prime}}(\hat{\mathfrak{g}})^{*}$, and expand $\Delta(f)$ as a series $\Delta(f)=\sum_{\sigma}\left(\mathfrak{F}_{\sigma} \cdot \Phi_{\sigma} \cdot \mathfrak{E}_{\sigma}\right) \otimes\left(\mathfrak{F}_{\sigma}^{\prime} \cdot \Phi_{\sigma}^{\prime} \cdot \mathfrak{E}_{\sigma}^{\prime}\right)$ so that $\Phi_{\sigma} \otimes \Phi_{\sigma}^{\prime} \neq \Phi_{\tau} \otimes \Phi_{\tau}^{\prime}\left(\in U_{0}^{M^{\prime *}} \widehat{\otimes} U_{0}^{M^{\prime *}}=\right.$ $\left.\left(U_{0}^{M^{\prime}} \otimes U_{0}^{M^{\prime}}\right)^{*}\right)$ for all $\sigma, \tau$, such that $\left(\mathfrak{F}_{\sigma}, \mathfrak{E}_{\sigma}, \mathfrak{F}_{\sigma}^{\prime}, \mathfrak{E}_{\sigma}^{\prime}\right) \neq\left(\mathfrak{F}_{\tau}, \mathfrak{E}_{\tau}, \mathfrak{F}_{\tau}^{\prime}, \mathfrak{E}_{\tau}^{\prime}\right)$ (this is always 
possible). As $f \in \mathcal{U}^{M^{\prime}}(\hat{\mathfrak{g}})$, then $\Delta(f)$ is integer-valued on $\mathcal{U}^{M^{\prime}}(\hat{\mathfrak{g}}) \otimes \mathcal{U}^{M^{\prime}}(\hat{\mathfrak{g}})$. Fix any $\bar{\sigma}$ : as above there exist suitable PBW-like monomials $\mathcal{E}_{\bar{\sigma}}, \mathcal{F}_{\bar{\sigma}}, \mathcal{E}_{\bar{\sigma}}^{\prime}, \mathcal{F}_{\bar{\sigma}}^{\prime}$ such that

$$
\left\langle\Delta(f),\left(\mathcal{E}_{\bar{\sigma}} \otimes \mathcal{E}_{\bar{\sigma}}^{\prime}\right) \cdot\left(L_{\nu} \otimes L_{\nu^{\prime}}\right) \cdot\left(\mathcal{F}_{\bar{\sigma}} \otimes \mathcal{F}_{\bar{\sigma}}^{\prime}\right)\right\rangle= \pm q^{z} \cdot\left\langle\phi_{\sigma} \otimes \phi_{\sigma}^{\prime}, L_{\nu} \otimes L_{\nu^{\prime}}\right\rangle
$$

for all $\nu, \nu^{\prime} \in M^{\prime}$ (for some $z \in \mathbb{Z}$ ); since $\Delta(f)$ is integer-valued, $\phi_{\bar{\sigma}} \otimes \phi_{\bar{\sigma}}^{\prime}$ is integervalued on $\mathcal{U}_{0}^{M^{\prime}} \otimes \mathcal{U}_{0}^{M^{\prime}}$, that is $\phi_{\bar{\sigma}} \otimes \phi_{\bar{\sigma}}^{\prime} \in\left(\mathcal{U}_{0}^{M^{\prime}} \otimes \mathcal{U}_{0}^{M^{\prime}}\right)^{*}=\mathcal{U}_{0}^{M^{\prime}} \widehat{ }^{*} \mathcal{U}_{0}^{M^{\prime}}$; but $\phi_{\bar{\sigma}} \otimes \phi_{\bar{\sigma}}^{\prime} \in$ $U_{0}^{M^{\prime *}} \otimes U_{0}^{M^{\prime *}}$, thus $\phi_{\bar{\sigma}}, \phi_{\bar{\sigma}}^{\prime} \in \mathcal{U}_{0}^{M^{\prime *}}$, q.e.d.

Finally, we have $1 \in \mathcal{U}^{M^{\prime}}(\hat{\mathfrak{g}})^{*}$, because $1:=\epsilon, \epsilon\left(\mathcal{U}^{M^{\prime}}(\hat{\mathfrak{g}})^{*}\right) \subseteq R$ because $\epsilon:=1^{*}$ and $1 \in \mathcal{U}^{M^{\prime}}(\hat{\mathfrak{g}})$, and $S\left(\mathcal{U}^{M^{\prime}}(\hat{\mathfrak{g}})^{*}\right)=\mathcal{U}^{M^{\prime}}(\hat{\mathfrak{g}})^{*}$ because $S:=S^{*}$ and $S\left(\mathcal{U}^{M^{\prime}}(\hat{\mathfrak{g}})\right)=\mathcal{U}^{M^{\prime}}(\hat{\mathfrak{g}})$. Thus $\mathcal{U}^{M^{\prime}}(\hat{\mathfrak{g}})^{*}$ is a formal Hopf subalgebra of $U_{q}^{M^{\prime}}(\hat{\mathfrak{g}})^{*}$, q.e.d.

Definition 4.10. We call $A^{M}$ the subalgebra of $U_{\leq}^{M} \otimes U_{\geq}^{P \infty}\left(\subset D_{M^{\prime}}{ }^{*}\right)$ generated by $\left\{F_{i} \otimes 1, L_{-\mu} \otimes L_{\mu}, 1 \otimes E_{i} \mid i \in I, \mu \in M\right\}$. Then we set

$$
\begin{aligned}
& \mathfrak{A}^{M}:=\left\{f \in A^{M} \mid\left\langle f, \mathfrak{U}^{M^{\prime}}(\hat{\mathfrak{g}})\right\rangle \subseteq R\right\}=A^{M} \cap \mathfrak{I}^{M} \\
& \mathcal{A}^{M}:=\left\{f \in A^{M} \mid\left\langle f, \mathcal{U}^{M^{\prime}}(\hat{\mathfrak{g}})\right\rangle \subseteq R\right\}=A^{M} \cap \mathcal{I}^{M} .
\end{aligned}
$$

\section{Lemma 4.11.}

(a) $\mathfrak{A}^{M}$ is an $R$-integer form of $A^{M}$, generated as an $R$-subalgebra by

$$
\left\{\bar{F}_{\alpha} \otimes 1, L_{-\mu} \otimes L_{\mu}, 1 \otimes \bar{E}_{\alpha} \mid \alpha \in \widetilde{\Phi}_{+}, \mu \in M\right\} .
$$

(b) $\mathcal{A}^{M}$ is an $R$-integer form of $A^{M}$, generated as an $R$-subalgebra by

$$
\left\{F_{i}^{(a)} \otimes 1,\left(\begin{array}{c}
L_{-\mu_{j}} \otimes L_{\mu_{j}} ; c \\
t
\end{array}\right), L_{\mu_{j}} \otimes L_{-\mu_{j}}, 1 \otimes E_{i}^{(d)} \mid i \in I, j \in I_{\infty} ; a, t, d \in \mathbb{N} ; c \in \mathbb{Z}\right\} .
$$

Proof. Definitions yield a linear isomorphism $\Phi_{M}: A^{M} \cong U_{-} \otimes U_{0}^{M} \otimes U_{+}$given by $\Phi_{M}: F_{i} \otimes 1 \mapsto F_{i} \otimes 1 \otimes 1, L_{-\mu} \otimes L_{\mu} \mapsto 1 \otimes L_{\mu} \otimes 1,1 \otimes E_{i} \mapsto 1 \otimes 1 \otimes E_{i} ;$ but this restricts to $\Phi_{M}: \mathcal{A}^{M} \stackrel{\cong}{\longrightarrow} \mathcal{U}_{-} \otimes \mathcal{U}_{0}^{M} \otimes \mathcal{U}_{+}, \Phi_{M}: \mathfrak{A}^{M} \stackrel{\cong}{\longrightarrow} \mathfrak{U}_{-} \otimes \mathfrak{U}_{0}^{M} \otimes \mathfrak{U}_{+}$, so $\S 3.3$ yields the claim.

4.12 Gradings. Recall (cf. $\S 2.1)$ that $U_{\geq}^{M}$ has a $Q_{+}$-grading $U_{\geq}^{M}=\oplus_{\alpha \in Q_{+}}\left(U_{\geq}^{M}\right)_{\alpha}$ given by decomposition in direct sum of weight spaces for the adjoint action of $U_{0}^{M}$; also $U_{\leq}^{M}$ has an analogous $Q_{-}$-grading. These are gradings of Hopf algebras (in the usual obvious sense), inherited by the integer forms, and DRT pairings respect them, that is e.g. $\pi\left(\left(U_{\leq}^{M}\right)_{\beta},\left(U_{\geq}^{M^{\prime}}\right)_{\gamma}\right)=0$ for all $\beta \in Q_{-}, \gamma \in Q_{+}$such that $\beta+\gamma \neq 0$.

The gradings of quantum Borel subalgebras induce a $Q$-grading of the Hopf algebra $D_{M}:=U_{\geq}^{M} \otimes U_{\leq}^{Q_{\infty}}$ (inherited by its quotient Hopf algebra $U_{q}^{M}(\hat{\mathfrak{g}})$ ), where the subspace 
$\left(U_{\geq}^{M}\right)_{\beta} \otimes\left(U_{\leq}^{Q \infty}\right)_{\gamma}$ has degree $\beta+\gamma$, and also a $Q$-grading of the subalgebra $U_{\geq}^{M} \otimes U_{\leq}^{Q_{\infty}}$ of $D_{M^{\prime}}{ }^{*}$; since $D_{M^{\prime}}{ }^{*}$ is a completion (via formal series) of this subalgebra, it inherits on its own sort of a "pseudograding", in the sense that every element of $D_{M^{\prime}}{ }^{*}$ is a (possibly infinite) sum of terms each of whom has a well-defined degree: namely, given $f \in D_{M^{\prime}}{ }^{*}$ with formal series expansion (cf. Remark 4.5) $f=\sum_{F, \phi, E} F \cdot \phi \cdot E$ (where $\phi \in\left(U_{0}^{M^{\prime}} \otimes U_{0}^{Q \infty}\right)^{*}$ and the $F$ 's and the E's are PBW monomials), we define the degree of its various summands by

$$
\operatorname{deg}(F \cdot \phi \cdot E):=\operatorname{deg}(F)+\operatorname{deg}(E)
$$

where $\operatorname{deg}\left(\prod_{\alpha \in \widetilde{\Phi}_{+}} F_{\alpha}^{f_{\alpha}}\right):=-\sum_{\alpha \in \widetilde{\Phi}_{+}} f_{\alpha} \alpha, \operatorname{deg}\left(\prod_{\alpha \in \widetilde{\Phi}_{+}} E_{\alpha}^{e_{\alpha}}\right):=\sum_{\alpha \in \widetilde{\Phi}_{+}} e_{\alpha} \alpha$ (this degree is again a weight for a suitable action of $U_{0}^{M}$ on $\left.U_{\leq}^{M} \otimes U_{\geq}^{P \infty}\right)$. Now $U_{\leq}^{M} \otimes U_{\geq}^{P \infty}$ is dense in $D_{M^{\prime}}{ }^{*}$, and the restriction of the pairing $D_{M^{\prime}}{ }^{*} \otimes D_{M^{\prime}} \rightarrow \mathbb{C}(q)$ to $\left(U_{\leq}^{M} \otimes U_{\geq}^{P_{\infty}}\right) \otimes$ $\left(U_{\geq}^{M^{\prime}} \otimes U_{\leq}^{Q \infty}\right)$ is nothing but $(\pi \otimes \bar{\pi}) \circ \tau_{2,3}$ (with $\left.\tau_{2,3}: x \otimes y \otimes z \otimes w \mapsto x \otimes z \otimes y \otimes w ;\right)$ therefore, since $\pi$ and $\bar{\pi}$ respect the gradings, also the pairing $D_{M^{\prime}}{ }^{*} \otimes D_{M^{\prime}} \rightarrow \mathbb{C}(q)$ respects the pseudogradings we are dealing with.

Finally, the pseudograding of $D_{M^{\prime}}{ }^{*}$ is compatible with the formal Hopf structure. For example, look at $S(x)$, for homogeneous $x \in D_{M^{\prime}}{ }^{*}$ : given homogeneous $y \in D_{M^{\prime}}$, we have $\langle S(x), y\rangle=\langle x, S(y)\rangle=\left\langle x, y^{\prime}\right\rangle$ where $y^{\prime}:=S(y)$ is homogeneous on its own of degree $\operatorname{deg}\left(y^{\prime}\right)=\operatorname{deg}(y)$ (for the grading of $D_{M^{\prime}}$ is compatible with the Hopf structure); therefore we have $\operatorname{deg}(S(x))=\operatorname{deg}(x)$, because

$$
\langle S(x), y\rangle \neq 0 \Longrightarrow \operatorname{deg}(y)=\operatorname{deg}\left(y^{\prime}\right)=\operatorname{deg}(x) \Longrightarrow S(x) \in\left(D_{M^{\prime}}{ }^{*}\right)_{\operatorname{deg}(x)} .
$$

4.13 Some umbral calculus. In this section we provide concrete information about the Hopf structure of our quantum formal groups. This will be especially important to define integer forms and speciale them at roots of 1 . To be short, we set

$$
F_{i}^{\otimes}:=F_{i} \otimes 1, \quad 1^{\otimes}:=1 \otimes 1, \quad E_{i}^{\otimes}:=1 \otimes E_{i}, \quad L_{\mu}^{\otimes}:=L_{-\mu} \otimes L_{\mu} \quad \forall i \in I, \mu \in M
$$

The counit $\epsilon: D_{M^{\prime}}{ }^{*} \rightarrow \mathbb{C}(q)$ is $\epsilon:=1^{*}$, hence $\epsilon\left(x^{*}\right):=\left\langle x^{*}, 1\right\rangle \forall x^{*} \in D_{M^{\prime}}{ }^{*}$; thus

$$
\epsilon\left(F_{i}^{\otimes}\right)=0, \quad \epsilon\left(L_{\mu}^{\otimes}\right)=1, \quad \epsilon\left(E_{i}^{\otimes}\right)=0 ;
$$

the elements above generate the algebra $j_{M}\left(U_{q}^{M^{\prime}}(\hat{\mathfrak{g}})^{*}\right)$ (in topological sense, cf. Proposition 4.6), hence (4.4) uniquely determines $\epsilon: j_{M}\left(U_{q}^{M^{\prime}}(\hat{\mathfrak{g}})^{*}\right) \longrightarrow \mathbb{C}(q)$.

The antipode of $D_{M^{\prime}}{ }^{*}$ is by definition the dual of the antipode of $D_{M^{\prime}}$, hence it is characterized by $\left\langle S\left(x^{*}\right), x\right\rangle=\left\langle x^{*}, S(x)\right\rangle$, for all $x^{*} \in D_{M^{\prime}}{ }^{*}, x \in D_{M^{\prime}}$. Now consider $F_{i}^{\otimes}=F_{i}^{f} \otimes 1 \in U_{\leq}^{M} \otimes U_{\geq}^{P \infty} \leq D_{M^{\prime}}{ }^{*}, f \in \mathbb{N}$ : it is homogeneous of degree $-f \alpha_{i}$, whence $S\left(F_{i}^{f} \otimes 1\right)$ has the same degree. Thus writing $S\left(F_{i}^{\otimes f} \otimes 1\right)$ as a series

$$
S\left(F_{i}^{\otimes}\right)=\sum_{\sigma} F_{\sigma} \cdot \Phi_{\sigma} \cdot E_{\sigma}
$$


we have $\operatorname{deg}\left(F_{\sigma} \cdot \Phi_{\sigma} \cdot E_{\sigma}\right):=\operatorname{deg}\left(F_{\sigma}\right)+\operatorname{deg}\left(E_{\sigma}\right)=-f \alpha_{i}$. Now, the pseudograding of $D_{M^{\prime}}{ }^{*}$ induces a pseudograding of $\mathfrak{I}^{M}$ too; hence, since $\mathfrak{I}^{M}$ is a formal Hopf subalgebra of $D_{M^{\prime}}{ }^{*}$ (Proposition 4.9), we can apply the same procedure and get

$$
S\left(\bar{F}_{i}^{\otimes f}\right)=\sum_{\sigma} \mathcal{F}_{\sigma} \cdot \varphi_{\sigma} \cdot \mathcal{E}_{\sigma}
$$

where $\varphi_{\sigma} \in \mathfrak{U}_{0}^{M^{\prime *}}$ and the $\mathcal{F}_{\sigma}$ 's, resp. $\mathcal{E}_{\sigma}$ 's, are PBW monomials of $\mathcal{U}_{-}$, resp. $\mathcal{U}_{+}$, such that $\operatorname{deg}\left(\mathcal{F}_{\sigma}\right)+\operatorname{deg}\left(\mathcal{E}_{\sigma}\right)=-f \alpha_{i}$. An entirely similar argument yields

$$
S\left(F_{i}^{\otimes(f)} \otimes 1\right)=\sum_{\sigma} \mathfrak{F}_{\sigma} \cdot \phi_{\sigma} \cdot \mathfrak{E}_{\sigma}
$$

where $\phi_{\sigma} \in \mathcal{U}_{0}^{M^{\prime *}}$ and the $\mathfrak{F}_{\sigma}$ 's, resp. $\mathfrak{E}_{\sigma}$, are PBW monomials of $\mathfrak{U}_{-}$, resp. $\mathfrak{U}_{+}$, such that $\operatorname{deg}\left(\mathfrak{F}_{\sigma}\right)+\operatorname{deg}\left(\mathfrak{E}_{\sigma}\right)=-f \alpha_{i}$.

Now, $\mathfrak{I}^{M}$ and $\mathcal{I}^{M}$ can be compared through the natural embedding $\mathcal{I}^{M} \cong \mathfrak{U}^{M^{\prime}}(\hat{\mathfrak{g}})^{*} \hookrightarrow$ $\mathcal{U}^{M^{\prime}}(\hat{\mathfrak{g}})^{*} \cong \mathfrak{I}^{M}$ (dual of $\left.\mathcal{U}^{M^{\prime}}(\hat{\mathfrak{g}}) \hookrightarrow \mathfrak{U}^{M^{\prime}}(\hat{\mathfrak{g}})\right)$; directly from definitions we get

$$
\begin{array}{clr}
\bar{F}_{\gamma}^{f}=\prod_{s=1}^{f}\left(q_{\gamma}^{s}-q_{\gamma}^{-s}\right) \cdot F_{\gamma}^{(f)}, & \bar{E}_{\gamma}^{e}=\prod_{s=1}^{e}\left(q_{\gamma}^{s}-q_{\gamma}^{-s}\right) \cdot E_{\gamma}^{(e)} & \forall \gamma \in \Phi_{+}^{\mathrm{re}} \\
\bar{F}_{(r \delta, i)}^{f}=\left(q_{i}^{r}-q_{i}^{-r}\right)^{f} \frac{f !}{r^{f}} \cdot F_{(r \delta, i)}^{(f)}, & \bar{E}_{(r \delta, i)}^{e}=\left(q_{i}^{r}-q_{i}^{-r}\right)^{e} \frac{e !}{r^{e}} \cdot E_{(r \delta, i)}^{(e)} & \forall(r \delta, i) \in \widetilde{\Phi}_{+}^{\mathrm{im}}
\end{array}
$$

thus comparing (4.5) and (4.6) we find

$$
\begin{gathered}
\mathfrak{F}_{\sigma} \cdot \phi_{\sigma} \cdot \mathfrak{E}_{\sigma} \in\left(\prod_{u=1}^{f}\left(q_{i}^{u}-q_{i}^{-u}\right)^{-1} \cdot \prod_{\beta, \gamma \in \Phi_{+}^{\mathrm{re}}} \prod_{r=1}^{f_{\beta}}\left(q_{\beta}^{r}-q_{\beta}^{-r}\right) \cdot \prod_{s=1}^{e_{\gamma}}\left(q_{\gamma}^{s}-q_{\gamma}^{-s}\right)\right. \\
\left.\cdot \prod_{(r \delta, i),(t \delta, j) \in \widetilde{\Phi}_{+}^{\mathrm{im}}}\left(q_{i}^{r}-q_{i}^{-r}\right)^{f_{(r \delta, i)}}\left(q_{j}^{s}-q_{j}^{-s}\right)^{e_{(s \delta, j)}}\right) \cdot \mathcal{I}^{M}
\end{gathered}
$$

for $\mathfrak{F}_{\sigma}=\prod_{\alpha \in \widetilde{\Phi}_{+}} F_{\alpha}^{\left(f_{h}\right)}, \mathfrak{E}_{\sigma}=\prod_{\alpha \in \widetilde{\Phi}_{+}} E_{\alpha}^{\left(e_{k}\right)}$. Similar remarks hold for the other generators of $\mathcal{A}^{M}$ : in particular, a first consequence is the following

Claim I: The series $S\left(F_{i}^{\otimes(f)}\right), S\left(\left(\begin{array}{c}L_{\mu_{i}}^{\otimes} ; c \\ t\end{array}\right)\right), S\left(L_{-\mu_{i}}^{\otimes}\right)$ and $S\left(E_{i}^{\otimes(e)}\right)$ are convergent in the $\left(q-q^{-1}\right) \mathcal{I}^{M}$-adic topology of $\mathcal{I}^{M}$; in particular, they are finite sums modulo $(q-1)$.

In principle, one can compute all the terms of these series up to any fixed order (in $\left.\left(q-q^{-1}\right)\right)$ : actually, we need to know them only up to the zeroth order. For $S\left(F_{i}^{\otimes}\right)$ the first term (call it $\mathbf{F}_{1}$ ), of order zero in the $\left(q-q^{-1}\right)$-adic expansion of $S\left(F_{i}^{\otimes}\right)$, 
corresponds to the terms $\mathfrak{F}_{\sigma} \cdot \phi_{\sigma} \cdot \mathfrak{E}_{\sigma}$ in (4.6) such that $\sum_{\alpha \in \widetilde{\Phi}_{+}}\left(f_{\alpha}+e_{\alpha}\right)=1$; on the other hand, these must have degree $\operatorname{deg}\left(\mathfrak{F}_{\sigma}\right)+\operatorname{deg}\left(\mathfrak{E}_{\sigma}\right)=-\alpha_{i}$ too, whence it is $\mathfrak{F}_{\sigma}=F_{i}^{\otimes}$ and $\mathfrak{E}_{\sigma}^{\otimes}=1$. Now, $\mathbf{F}_{1}$ takes non-zero values only on PBW monomials of type $\bar{E}_{i} \cdot L_{\nu},\left(\nu \in M^{\prime}\right)$; so let $V_{1, i}$ be the free $\mathcal{U}_{0}^{M^{\prime}}$-module with basis $\left.\left\{\bar{E}_{i}\right\}\right)$ : direct computation shows that $\mathbf{F}_{1}+q_{i}^{-2} \cdot F_{i}^{\otimes} L_{-\alpha_{i}}^{\otimes}$ is zero in $V_{1, i}^{*}$, therefore

$$
S\left(F_{i}^{\otimes}\right) \equiv-q^{-2} \cdot F_{i}^{\otimes} L_{-\alpha_{i}}^{\otimes} \quad \bmod \left(q-q^{-1}\right) \cdot \mathcal{I}^{M}
$$

Similar arguments give

$$
\begin{array}{rr}
S\left(\left(\begin{array}{c}
L_{\mu_{i}}^{\otimes} ; 0 \\
1
\end{array}\right)\right) \equiv-L_{\mu_{i}}^{\otimes}\left(\begin{array}{c}
L_{\mu_{i}}^{\otimes} ; 0 \\
1
\end{array}\right) & \bmod \left(q-q^{-1}\right) \cdot \mathcal{I}^{M} \\
S\left(E_{i}^{\otimes}\right) \equiv-q^{+2} \cdot L_{-\alpha_{i}}^{\otimes} E_{i}^{\otimes} & \bmod \left(q-q^{-1}\right) \cdot \mathcal{I}^{M}
\end{array}
$$

As for the coproduct $\Delta: D_{M^{\prime}}{ }^{*} \rightarrow D_{M^{\prime}}{ }^{*} \widehat{\otimes} D_{M^{\prime}}{ }^{*}$, it is the dual of the product of $D_{M^{\prime}}$, hence it is characterized by $\left\langle\Delta\left(x^{*}\right), y \otimes z\right\rangle=\left\langle x^{*}, y \cdot z\right\rangle$. The same kind of procedure used for $S$ may be applied in the present case. Thus for instance, if

$$
\Delta\left(F_{i}^{\otimes(f)}\right)=\sum_{\sigma, \tau}\left(\mathfrak{F}_{\sigma} \cdot \phi_{\sigma} \cdot \mathfrak{E}_{\sigma}\right) \otimes\left(\mathfrak{F}_{\tau} \cdot \phi_{\tau} \cdot \mathfrak{E}_{\tau}\right)
$$

is the series expansion of $\Delta\left(F_{i}^{\otimes(f)}\right)$ as in Proposition $4.9(b)\left(\right.$ via $\left.\mathcal{I}^{M} \cong \mathcal{U}^{M^{\prime}}(\hat{\mathfrak{g}})^{*}\right)$, then

$$
\begin{aligned}
& \left(\mathfrak{F}_{\sigma} \cdot \phi_{\sigma} \cdot \mathfrak{E}_{\sigma}\right) \otimes\left(\mathfrak{F}_{\sigma^{\prime}} \cdot \phi_{\sigma^{\prime}} \cdot \mathfrak{E}_{\sigma^{\prime}}\right) \in\left(\prod_{u=1}^{f}\left(q_{i}^{u}-q_{i}^{-u}\right)^{-1} \cdot\right. \\
& \cdot \prod_{\beta, \gamma \in \Phi_{+}^{\mathrm{re}}} \prod_{r=1}^{f_{\beta}}\left(q_{\beta}^{r}-q_{\beta}^{-r}\right) \cdot \prod_{s=1}^{e_{\gamma}}\left(q_{\gamma}^{s}-q_{\gamma}^{-s}\right) \cdot \prod_{(r \delta, i),(t \delta, j) \in \widetilde{\Phi}_{+}^{\mathrm{im}}}\left(q_{i}^{r}-q_{i}^{-r}\right)^{f_{(r \delta, i)}}\left(q_{j}^{s}-q_{j}^{-s}\right)^{e_{(s \delta, j)}} \cdot \quad(4.9) \\
& \left.\cdot \prod_{\beta, \gamma \in \Phi_{+}^{\mathrm{re}}} \prod_{r=1}^{f_{\beta}^{\prime}}\left(q_{\beta}^{r}-q_{\beta}^{-r}\right) \cdot \prod_{s=1}^{e_{\gamma}^{\prime}}\left(q_{\gamma}^{s}-q_{\gamma}^{-s}\right) \cdot \prod_{(r \delta, i),(t \delta, j) \in \widetilde{\Phi}_{+}^{\mathrm{im}}}\left(q_{i}^{r}-q_{i}^{-r}\right)^{f_{(r \delta, i)}^{\prime}}\left(q_{j}^{s}-q_{j}^{-s}\right)^{e_{(s \delta, j)}^{\prime}}\right) \cdot \mathcal{I}^{M}
\end{aligned}
$$

for $\mathfrak{F}_{\sigma}=\prod_{\alpha \in \widetilde{\Phi}_{+}} F_{\alpha}^{\left(f_{h}\right)}, \mathfrak{E}_{\sigma}=\prod_{\alpha \in \widetilde{\Phi}_{+}} E_{\alpha}^{\left(e_{k}\right)}, \mathfrak{F}_{\sigma^{\prime}}=\prod_{\alpha \in \widetilde{\Phi}_{+}} F_{\alpha}^{\left(f_{h}^{\prime}\right)}, \mathfrak{E}_{\sigma^{\prime}}=\prod_{\alpha \in \widetilde{\Phi}_{+}} E_{\alpha}^{\left(e_{k}^{\prime}\right)}$ Similar remarks hold for the other generators of $\mathcal{A}^{M}$. As a first consequence, we have:

Claim II: The series $\Delta\left(F_{i}^{\otimes(f)}\right), \Delta\left(\left(\begin{array}{c}L_{\mu_{i}}^{\otimes} ; c \\ t\end{array}\right)\right), \Delta\left(L_{-\mu_{i}}^{\otimes}\right)$ and $\Delta\left(E_{i}^{\otimes(e)}\right)$ are convergent in the $\left(q-q^{-1}\right) \cdot\left(\mathcal{I}^{M} \widehat{\otimes} \mathcal{I}^{M}\right)$-adic topology of $\mathcal{I}^{M} \widehat{\otimes} \mathcal{I}^{M}$; in particular, they are finite sums modulo $(q-1)$. 
Direct computation gives us the following congruences modulo $\left(q-q^{-1}\right)^{2}$ :

$$
\begin{aligned}
& \Delta\left(F_{i}^{\otimes}\right) \equiv F_{i}^{\otimes} \otimes 1^{\otimes}+1^{\otimes} \otimes F_{i}^{\otimes}+\left(q_{i}-1\right) \cdot\left(\begin{array}{c}
L_{\alpha_{i}}^{\otimes} ; 0 \\
1
\end{array}\right) \otimes F_{i}^{\otimes}+ \\
& +\left(q_{i}-q_{i}^{-1}\right)^{-1} \cdot \sum C_{\alpha, \beta}^{i,+}\left(q_{\alpha}-q_{\alpha}^{-1}\right)\left(q_{\beta}-q_{\beta}^{-1}\right) \cdot L_{\alpha_{i}}^{\otimes} E_{\alpha}^{\otimes} \otimes F_{\beta}^{\otimes} \quad \bmod \left(q-q^{-1}\right)^{2} \\
& \begin{array}{c}
\alpha, \beta \in \widetilde{\Phi}_{+} \\
p(\alpha)-p(\beta)=-\alpha_{i}
\end{array} \\
& \Delta\left(\left(\begin{array}{c}
L_{\mu_{i}}^{\otimes} ; 0 \\
1
\end{array}\right)\right) \equiv\left(\begin{array}{c}
L_{\mu_{i}}^{\otimes} ; 0 \\
1
\end{array}\right) \otimes 1^{\otimes}+1^{\otimes} \otimes\left(\begin{array}{c}
L_{\mu_{i}}^{\otimes} ; 0 \\
1
\end{array}\right)+\left(q_{i}-1\right) \cdot\left(\begin{array}{c}
L_{\mu_{i}}^{\otimes} ; 0 \\
1
\end{array}\right) \otimes\left(\begin{array}{c}
L_{\mu_{i}}^{\otimes} ; 0 \\
1
\end{array}\right)+ \\
& +(2)_{q^{-1}}^{2}\left(d_{i}\right)_{q}^{-1} \cdot \sum(q-1) C_{\alpha, \beta}\left[d_{\gamma}\right]_{q}\left[\left(\mu_{i} \mid \gamma\right)\right]_{q} \cdot L_{\mu_{i}}^{\otimes} E_{\alpha}^{\otimes} \otimes F_{\beta}^{\otimes} L_{\mu_{i}}^{\otimes} \bmod \left(q-q^{-1}\right)^{2} \\
& \begin{array}{c}
\alpha, \beta \in \widetilde{\Phi}_{+} \\
p(\alpha)-p(\beta)=0
\end{array} \\
& \Delta\left(E_{i}^{\otimes}\right) \equiv 1^{\otimes} \otimes E_{i}^{\otimes}+E_{i}^{\otimes} \otimes 1^{\otimes}+\left(q_{i}-1\right) \cdot E_{i}^{\otimes} \otimes\left(\begin{array}{c}
L_{\alpha_{i}}^{\otimes} ; 0 \\
1
\end{array}\right)- \\
& -\left(q_{i}-q_{i}^{-1}\right)^{-1} \cdot \sum_{\substack{\alpha, \beta \in \widetilde{\Phi}_{+} \\
p(\alpha)-p(\beta)=+\alpha_{i}}} C_{\alpha, \beta}^{i,-}\left(q_{\alpha}-q_{\alpha}^{-1}\right)\left(q_{\beta}-q_{\beta}^{-1}\right) \cdot E_{\alpha}^{\otimes} \otimes F_{\beta}^{\otimes} L_{\alpha_{i}}^{\otimes} \quad \bmod \left(q-q^{-1}\right)^{2}
\end{aligned}
$$

where the $C_{\alpha, \beta}^{i, \pm}$, s are given by the equations $\pi_{i}^{-}\left(\left[\dot{F}_{\alpha}, \dot{E}_{\beta}\right]\right)=C_{\alpha, \beta}^{i,-} \cdot F_{i}, \pi_{i}^{+}\left(\left[\dot{F}_{\alpha}, \dot{E}_{\beta}\right]\right)=$ $C_{\alpha, \beta}^{i,+} \cdot E_{i}\left(\pi_{i}^{-}: U_{q}^{Q}(\hat{\mathfrak{g}}) \rightarrow \mathbb{C}(q) \cdot F_{i}\right.$ and $\pi_{i}^{+}: U_{q}^{Q}(\hat{\mathfrak{g}}) \rightarrow \mathbb{C}(q) \cdot E_{i}$ being the canonical maps $)$ and the $C_{\alpha, \beta}$ 's by the equation $\left[\dot{F}_{\alpha}, \dot{E}_{\beta}\right]=C_{\alpha, \beta} \frac{L_{p(\alpha)}-L_{p(\alpha)}^{-1}}{q_{\beta}-q_{\beta}^{-1}}$ : here $\dot{F}_{\gamma}$, resp. $\dot{E}_{\gamma}$, is the dual, resp. minus the dual, of $E_{\gamma}$, resp. $F_{\gamma}$, with respect to $\pi$, resp. $\bar{\pi}$.

\section{$\S 5$ The quantum group $U_{q}^{M}(\hat{\mathfrak{h}})$}

5.1 The quantum enveloping algebra $U_{q}^{M}(\hat{\mathfrak{h}})$. The results of $\S 4$ can be given an axiomatic form: to this end, we introduce a new object $U_{q}^{M}(\hat{\mathfrak{h}})$ which is with respect to $U(\hat{\mathfrak{h}})$ what $U_{q}^{M}(\hat{\mathfrak{g}})$ is for $U(\hat{\mathfrak{g}})$. Here $M$ is a fixed lattice as in $\S 2.1$.

We define $\hat{\mathbf{H}}_{M}$ to be the associative $\mathbb{C}(q)$-algebra with 1 with generators $F_{i}, L_{\mu}$, $E_{i}$ for all $\lambda \in M, i \in I$, and relations (for all $\mu, \nu \in M, i, j \in I, i \neq j$ )

$$
\begin{gathered}
L_{0}=1, \quad L_{\mu} L_{\nu}=L_{\mu+\nu}, \quad E_{i} F_{j}-F_{j} E_{i}=0 \\
L_{\mu} F_{j}=q^{\left(\alpha_{j} \mid \mu\right)} F_{j} L_{\mu}, \quad \sum_{k=0}^{1-a_{i j}}(-1)^{k}\left[\begin{array}{c}
1-a_{i j} \\
k
\end{array}\right]_{q_{i}} F_{i}^{1-a_{i j}-k} F_{j} F_{i}^{k}=0 \\
L_{\mu} E_{j}=q^{\left(\alpha_{j} \mid \mu\right)} E_{j} L_{\mu}, \quad \sum_{k=0}^{1-a_{i j}}(-1)^{k}\left[\begin{array}{c}
1-a_{i j} \\
k
\end{array}\right]_{q_{i}} E_{i}^{1-a_{i j}-k} E_{j} E_{i}^{k}=0 .
\end{gathered}
$$


We also use notation $M_{i}:=L_{\mu_{i}}\left(i \in I_{\infty}\right)$, where $\left\{\mu_{i} \mid i \in I_{\infty}\right\}$ is a fixed $\mathbb{Z}$-basis of $M$.

Define $\mathbb{N}^{\widetilde{\Phi}_{+}}$to be the set of all functions $f: \widetilde{\Phi}_{+} \rightarrow \mathbb{N}$ such that $f(\alpha)=0$ for almost all $\alpha \in \widetilde{\Phi}_{+}$. For any $\phi:=\left(f_{\alpha}\right)_{\alpha \in \widetilde{\Phi}_{+}} \in \mathbb{N}^{\widetilde{\Phi}_{+}}, \eta:=\left(e_{\alpha}\right)_{\alpha \in \widetilde{\Phi}_{+}} \in \mathbb{N}^{\widetilde{\Phi}_{+}}$, set $F_{\phi}:=\prod_{\alpha \in \widetilde{\Phi}_{+}} F_{\alpha}^{f_{\alpha}}, E_{\eta}:=\prod_{\alpha \in \widetilde{\Phi}_{+}} E_{\alpha}^{e_{\alpha}}$, where the products are meant to be ordered, like in $\S 2$. We shall also use a similar notation when dealing with PBW monomials of $\mathfrak{U}_{ \pm}$or of $\mathcal{U}_{ \pm}$, e.g. $\mathfrak{F}_{\phi}:=\prod_{\alpha \in \widetilde{\Phi}_{+}} F_{\alpha}^{\left(f_{\alpha}\right)}, \mathcal{E}_{\eta}:=\prod_{\alpha \in \widetilde{\Phi}_{+}} \bar{E}_{\alpha}^{e_{\alpha}}$. Moreover, for any $\tau \in \mathbb{N}^{I_{\infty}}=\mathbb{N}^{n+2}$ set $B_{\tau}:=u_{\tau}^{*}$ (the element of the pseudobasis $\mathcal{B}_{M}$ constructed in Lemma 4.4(b).

We define $U_{q}^{M}(\hat{\mathfrak{h}})$ to be the completion of $\hat{\mathbf{H}}_{M}$ by means of formal series (i.e. infinite linear combinations), with coefficients in $\mathbb{C}(q)$, in the elements of the set

$$
\mathbb{B}_{M}:=\left\{F_{\phi} \cdot B_{\tau} \cdot E_{\eta} \mid \phi, \eta \in \mathbb{N}^{\widetilde{\Phi}_{+}}, \tau \in \mathbb{N}^{I_{\infty}}\right\} .
$$

Thus $U_{q}^{M}(\hat{\mathfrak{h}})$ is the completion of $\hat{\mathbf{H}}_{M}$ with respect to the topology (of $\hat{\mathbf{H}}_{M}$ ) for which a fundamental system of neighborhoods of 0 is the set of vector subspaces of $\hat{\mathbf{H}}_{M}$ which contain almost all the elements of $\mathbb{B}_{M}$, and the set $\mathbb{B}_{M}$ is a pseudobasis of $U_{q}^{M}(\hat{\mathfrak{h}})$. Roughly speaking, $U_{q}^{M}(\hat{\mathfrak{h}})$ is an algebra of (non-commutative) formal series with the (5.1) as commutation rules. Finally, thanks to Lemma 4.4, we can identify $U_{q}^{M}(\hat{\mathfrak{h}})$ with the space of formal series in the $F_{\alpha}$ 's, $E_{\alpha}$ 's, $\left(\alpha \in \widetilde{\Phi}_{+}\right)$with coefficients in $U_{0}^{M^{\prime \prime *}}$.

From $\S 4$ we can explicitely realize $U_{q}^{M}(\hat{\mathfrak{h}})$ and endow it with a Hopf structure: in fact, the definition of $U_{q}^{M}(\hat{\mathfrak{h}})$ is just a presentation of $U_{q}^{M^{\prime}}(\hat{\mathfrak{g}})^{*}$, as the following shows:

Theorem 5.2. There exists an isomorphism of topological $\mathbb{C}(q)$-algebras

$$
\nu_{M}: U_{q}^{M}(\hat{\mathfrak{h}}) \stackrel{\cong}{\longrightarrow} j_{M}\left(U_{q}^{M^{\prime}}(\hat{\mathfrak{g}})^{*}\right)
$$

given by $\quad F_{i} \mapsto F_{i}^{\otimes}:=F_{i} \otimes 1, \quad L_{\mu} \mapsto L_{\mu}^{\otimes}:=L_{-\mu} \otimes L_{\mu}, \quad E_{i} \mapsto E_{i}^{\otimes}:=1 \otimes E_{i}$.

Then the pull-back of the formal Hopf structure of $j_{M}\left(U_{q}^{M^{\prime}}(\hat{\mathfrak{g}})^{*}\right)$ defines a formal Hopf structure on $U_{q}^{M}(\hat{\mathfrak{h}})$, so that $\nu_{M}$ and $j_{M}{ }^{-1} \circ \nu_{M}$ are isomorphisms of formal Hopf algebras.

Proof. By construction $\hat{\mathbf{H}}_{M} \cong U_{-} \otimes U_{0}^{M} \otimes U_{+} \cong A^{M}\left(\subseteq j_{M}\left(U_{q}^{M^{\prime}}(\hat{\mathfrak{g}})^{*}\right)\right)$ as vector spaces; now $F_{i} \otimes 1, L_{-\mu} \otimes L_{\mu}, 1 \otimes E_{i} \in U_{\leq}^{M} \otimes U_{\geq}^{P \infty}$, hence comparing (5.1) and (2.1) we see that formulas above gives a well-defined isomorphism of algebras $\nu_{M}: \hat{\mathbf{H}}_{M} \stackrel{\cong}{\rightrightarrows} A^{M}$. Moreover, $A^{M}$ contains a pseudobasis $\mathbf{B}_{M}$ of $j_{M}\left(U_{q}^{M^{\prime}}(\hat{\mathfrak{g}})^{*}\right)$ (cf. Lemma 4.4, Proposition 4.6, and Remark 4.7) such that $\nu_{M}\left(\mathbb{B}_{M}\right)=\mathbf{B}_{M}$, hence $\nu_{M}$ continuosly extends, in a unique way, to an isomorphism of topological algebras $\nu_{M}: U_{q}^{M}(\hat{\mathfrak{h}}) \stackrel{\cong}{\longrightarrow} j_{M}\left(U_{q}^{M^{\prime}}(\hat{\mathfrak{g}})^{*}\right)$, q.e.d. 
$\underline{\text { Remark: }}$ For "restricted" specializations at roots of 1 the algebra $U_{q}^{M}(\hat{\mathfrak{h}})$ is too big; in order to get a reasonable $R$-integer form, it turns out to be necessary to impose two bounding conditions. The first condition is on the size of the "toral part" of an element in $U_{q}^{M}(\hat{\mathfrak{h}})$ : we tackle it in $\S \S 5.4-5$ below; the second one is on the behaviour of an element in $U_{q}^{M}(\hat{\mathfrak{h}})$ (as a series), and is inspired by (4.7) and (4.9): we deal with it in $\S \S 5.7-9$.

Hereafter, we freely use the term pseudobasis to mean a topological basis of a topological module, so that any element in the module has a unique expansion as a series in the elements of the pseudobasis.

Definition 5.3. We define the subset $\Omega_{M}$ of $U_{q}^{M}(\hat{\mathfrak{h}})$ to be

$$
\Omega_{M}:=\left\{x=\sum_{\sigma} F_{\sigma} \cdot \Phi_{\sigma} \cdot E_{\sigma} \in U_{q}^{M}(\hat{\mathfrak{h}}) \mid \Phi_{\sigma} \in U_{0}^{M}, \forall \sigma\right\}
$$

(where $x=\sum_{\sigma} F_{\sigma} \cdot \Phi_{\sigma} \cdot E_{\sigma}$ is the expansion of $x$ as a series with coefficients in $U_{0}^{M^{\prime *}}$ ).

\section{Lemma 5.4.}

(a) The set of all products $F \cdot L \cdot E$ in which $F$, resp. $L$, resp. $E$, is any element of a fixed $P B W$ basis of $U_{-}$, resp. $U_{0}^{M}$, resp. $U_{+}$, is a pseudobasis of $\Omega_{M}$.

(b) $\Omega_{M}$ is a formal Hopf subalgebra of $U_{q}^{M}(\hat{\mathfrak{h}})$.

Proof. Claim (a) follows from definitions. As for (b), it is clear that $\Omega_{M}$ is a subalgebra of $U_{q}^{M}(\hat{\mathfrak{h}})$. Now we show that it is also closed for the antipode and the coproduct.

Let $x=\sum_{\tau} F_{\tau}^{\prime} \cdot \Phi_{\tau}^{\prime} \cdot E_{\tau}^{\prime} \in \Omega_{M}$ : then $\Phi_{\tau}^{\prime}=\sum_{\mu \in M} c_{\tau, \mu} L_{\mu}$ with $c_{\tau, \mu} \neq 0$ for finitely many $\mu$. Let $S(x)=\sum_{\sigma} F_{\sigma} \cdot \Phi_{\sigma} \cdot E_{\sigma}$ : for any fixed $\bar{\sigma}$, we must prove that $\Phi_{\bar{\sigma}} \in U_{0}^{M}$ $\left(\subseteq U_{0}^{M^{\prime *}}\right)$, so that $S\left(\Omega_{M}\right)=\Omega_{M}$; to this end, we identify $U_{q}^{M}(\hat{\mathfrak{h}}) \cong U_{q}^{M^{\prime}}(\hat{\mathfrak{g}})^{*}$ (cf. Theorem 5.2). By Lemma 2.5 there exists two PBW monomials $\dot{\mathcal{E}}_{\bar{\sigma}}$ and $\dot{\mathcal{F}}_{\bar{\sigma}}$ such that

$$
\left\langle S(x), \dot{\mathcal{E}}_{\bar{\sigma}} \cdot y \cdot \dot{\mathcal{F}}_{\bar{\sigma}}\right\rangle=\left\langle F_{\sigma} \cdot \Phi_{\sigma} \cdot E_{\sigma}, \dot{\mathcal{E}}_{\bar{\sigma}} \cdot y \cdot \dot{\mathcal{F}}_{\bar{\sigma}}\right\rangle=\left\langle F_{\bar{\sigma}}, \dot{\mathcal{E}}_{\bar{\sigma}}\right\rangle \cdot\left\langle E_{\bar{\sigma}}, \dot{\mathcal{F}}_{\bar{\sigma}}\right\rangle \cdot \Phi_{\bar{\sigma}}\left(y \cdot L_{\alpha}\right)
$$

for all $y \in U_{0}^{M^{\prime}}$, with $\alpha:=s\left(F_{\bar{\sigma}}\right)+s\left(E_{\bar{\sigma}}\right)$ and $c_{\bar{\sigma}}:=\left\langle F_{\bar{\sigma}}, \dot{\mathcal{E}}_{\bar{\sigma}}\right\rangle \cdot\left\langle E_{\bar{\sigma}}, \dot{\mathcal{F}}_{\bar{\sigma}}\right\rangle \neq 0$ : in other words, $\Phi_{\bar{\sigma}}=\left.c_{\bar{\sigma}}{ }^{-1} \cdot\left(\left(L_{-\alpha} \cdot \dot{\mathcal{F}}_{\bar{\sigma}}\right) \triangleright S(x) \triangleleft \dot{\mathcal{E}}_{\bar{\sigma}}\right)\right|_{U_{0}^{M^{\prime}}} \quad($ where $\triangleleft$ and $\triangleright$ denote standard left and right action, cf. [DL], §1.4), hence we have to study $\left\langle S(x), \dot{\mathcal{E}}_{\bar{\sigma}} \cdot y \cdot L_{-\alpha} \dot{\mathcal{F}}_{\bar{\sigma}}\right\rangle$ as a function of $y \in U_{0}^{M^{\prime}}$; by linearity we can assume $y=L_{\nu}, \nu \in M^{\prime}$. By definition, $\left\langle S(x), \dot{\mathcal{E}}_{\bar{\sigma}} \cdot y \cdot L_{-\alpha} \dot{\mathcal{F}}_{\bar{\sigma}}\right\rangle=\left\langle x, S\left(\dot{\mathcal{E}}_{\bar{\sigma}} \cdot y L_{-\alpha} \cdot \dot{\mathcal{F}}_{\bar{\sigma}}\right)\right\rangle ;$ to compute the latter we have to "straighten" $S\left(\dot{\mathcal{E}}_{\bar{\sigma}} \cdot y \cdot L_{-\alpha} \dot{\mathcal{F}}_{\bar{\sigma}}\right)$, i.e. to write it in terms of a PBW basis of $U_{-} \otimes U_{0}^{M^{\prime}} \otimes U_{+}$.

Since $S\left(\dot{\mathcal{E}}_{\bar{\sigma}} \cdot y \cdot L_{-\alpha} \dot{\mathcal{F}}_{\bar{\sigma}}\right)=S\left(L_{-\alpha} \dot{\mathcal{F}}_{\bar{\sigma}}\right) \cdot S(y) \cdot S\left(\dot{\mathcal{E}}_{\bar{\sigma}}\right)$, let us consider the various factors. First, $S\left(L_{-\alpha} \dot{\mathcal{F}}_{\bar{\sigma}}\right) \in U_{\leq}^{M^{\prime}}$, and $S\left(L_{-\alpha} \dot{\mathcal{F}}_{\bar{\sigma}}\right)$ does not depend on $y$. Second, $S(y)=S\left(L_{\nu}\right)=L_{-\nu}$. Third, $S\left(\dot{\mathcal{E}}_{\bar{\sigma}}\right) \in U_{\geq}^{M^{\prime}}$, and $S\left(\dot{\mathcal{E}}_{\bar{\sigma}}\right)$ does not depend on $y$. 
Now we straighten the product. Commuting $S\left(L_{-\alpha} \dot{\mathcal{F}}_{\bar{\sigma}}\right)$ and $S(y)=L_{-\nu}$ produces a coefficient $q^{-\left(\nu \mid \beta_{\bar{\sigma}}\right)}=\left\langle L_{-\beta_{\bar{\sigma}}}, L_{\nu}\right\rangle_{\bar{\pi}}$ in which $\beta_{\bar{\sigma}} \in Q_{-}$is the weight of $S\left(\dot{\mathcal{F}}_{\bar{\sigma}}\right)$. Straightening the product $S\left(L_{-\alpha} \dot{\mathcal{F}}_{\bar{\sigma}}\right) \cdot S\left(\dot{\mathcal{E}}_{\bar{\sigma}}\right)$ produces a sum $\sum_{k} x_{k}$ of terms which do not depend on $y$. Straightening the product $S(y)=L_{-\nu} \cdot \sum_{k} x_{k}$ produces for each term $x_{k}$ a coefficient $q^{-\left(\nu \mid \gamma_{\bar{\sigma}, k}\right)}=\left\langle L_{-\gamma_{\bar{\sigma}, k}}, L_{\nu}\right\rangle_{\bar{\pi}}$, where $\gamma_{\bar{\sigma}, k} \in Q_{+}$is the weight of the "positive" part $x_{k}^{+}$of $x_{k}$ (with respect to the triangular decomposition).

Therefore $\left\langle x, S\left(\dot{\mathcal{E}}_{\bar{\sigma}} \cdot y \cdot L_{-\alpha} \dot{\mathcal{F}}_{\bar{\sigma}}\right)\right\rangle$ depends on $y$ according to the functions $L_{-\beta_{\bar{\sigma}}}$, $L_{-\gamma_{\bar{\sigma}, k}}$, and $\Phi_{\tau}^{\prime} \circ S$ : to be precise, $\Phi_{\bar{\sigma}}=\left.\left(L_{-\alpha} \dot{\mathcal{F}}_{\bar{\sigma}} \triangleright S(x) \triangleleft \dot{\mathcal{E}}_{\bar{\sigma}}\right)\right|_{U_{0}^{M^{\prime}}}$ is a linear combination of functions of type $L_{-\beta_{\bar{\sigma}}} \cdot\left(\Phi_{\tau}^{\prime} \circ S\right) \cdot L_{-\gamma_{\bar{\sigma}, k}}=\sum_{\mu \in M} c_{\tau, \mu} L_{-\mu-\beta_{\bar{\sigma}}-\gamma_{\bar{\sigma}, k}}$, so $\Phi_{\bar{\sigma}} \in U_{0}^{M}$, q.e.d. An entirely analogous procedure — slightly simpler indeed — works for comultiplication, thus proving that $\Delta\left(\Omega_{M}\right) \subseteq \Omega_{M} \widehat{\otimes} \Omega_{M}$. The claim follows.

Now we start introducing integer forms of $U_{q}^{M}(\hat{\mathfrak{h}})$ and proving their first properties.

Definition 5.5. We define $\hat{\mathcal{H}}_{M}$ to be the $R$-subalgebra of $U_{q}^{M}(\hat{\mathfrak{h}})$ generated by $\left\{\bar{F}_{\alpha}, L_{\mu}, \bar{E}_{\alpha} \mid \alpha \in \widetilde{\Phi}_{+} ; \mu \in M\right\}$, and $\mathcal{U}^{M}(\hat{\mathfrak{h}})$ to be its closure in $U_{q}^{M}(\hat{\mathfrak{h}})$.

Theorem 5.6. $\mathcal{U}^{M}(\hat{\mathfrak{h}})$ is an $R$-integer form (in topological sense) of $U_{q}^{M}(\hat{\mathfrak{h}})$, as a formal Hopf algebra, with $R$-pseudobasis

$$
\widetilde{\mathbb{B}}_{M}:=\left\{Y_{\phi, \tau, \eta} \mid \tau \in \mathbb{N}^{I_{\infty}} ; \phi, \eta \in \mathbb{N}^{\widetilde{\Phi}_{+}}\right\}=\left\{\mathcal{F}_{\phi} \cdot B_{\tau} \cdot \mathcal{E}_{\eta} \mid \tau \in \mathbb{N}^{I_{\infty}} ; \phi, \eta \in \mathbb{N}^{\widetilde{\Phi}_{+}}\right\}
$$

where $Y_{\phi, \tau, \eta}:=\mathcal{F}_{\phi} \cdot B_{\tau} \cdot \mathcal{E}_{\eta} ;$ in particular $\nu_{M}\left(\mathcal{U}^{M}(\hat{\mathfrak{h}})\right)=j_{M}\left(\mathfrak{U}^{M^{\prime}}(\hat{\mathfrak{g}})^{*}\right)=: \mathfrak{I}^{M}$.

Proof. By construction $\widetilde{\mathbb{B}}_{M} \subseteq \mathcal{U}^{M}(\hat{\mathfrak{h}})$, so the claim follows directly from $\S 5.1$.

Lemma 5.7. Let $\Omega_{M}^{\vee}:=\Omega_{M} \cap \nu_{M}^{-1}\left(\mathcal{I}^{M}\right)$; then $\Omega_{M}^{\vee}$ is the set of all $x \in U_{q}^{M}(\hat{\mathfrak{h}})$ which have a series expansion $x=\sum_{\mathfrak{F}, \mathfrak{E}} \mathfrak{F} \cdot \phi_{\mathfrak{F}, \mathfrak{E}} \cdot \mathfrak{E}$ where the $\mathfrak{F} \in \mathfrak{U}_{-}$and $\mathfrak{E} \in \mathfrak{U}_{+}$are $P B W$ monomials and $\phi_{\mathfrak{F}, \mathfrak{E}} \in \mathfrak{U}_{0}^{M}$ for all $\mathfrak{F}$, $\mathfrak{E}$.

Proof. Trivial from definitions and Proposition 4.9(b).

\section{Definition 5.8.}

(a) We define $\hat{\mathfrak{H}}_{M}$ to be the $R$-subalgebra of $U_{q}^{M}(\hat{\mathfrak{h}})$ generated by the set $\left\{F_{i}^{(f)},\left(\begin{array}{c}M_{j} ; c \\ t\end{array}\right), M_{j}^{-1}, E_{i}^{(e)} \mid f, c, t, e \in \mathbb{N} ; j \in I_{\infty}\right\}$.

(b) We define $\mathfrak{U}^{M}(\hat{\mathfrak{h}})$ to be the subset of all elements $x$ in $\Omega_{M}^{\vee}$ whose series expansion $x=\sum_{\varphi, \eta \in \mathbb{N}^{\Phi}} \mathfrak{F}_{\varphi} \cdot \phi_{\varphi, \eta} \cdot \mathfrak{E}_{\eta}$ (with notation of $\S 5.1$ ) is such that, for some 
$p(x) \in \mathbb{Z}\left[q, q^{-1}\right]$,

$$
\begin{gathered}
\phi_{\varphi, \eta} \in p(x)^{-1} \cdot\left(\prod_{\beta, \gamma \in \Phi_{+}^{\mathrm{re}}} \prod_{r=1}^{f_{\beta}}\left(q_{\beta}^{r}-q_{\beta}^{-r}\right) \cdot \prod_{s=1}^{e_{\gamma}}\left(q_{\gamma}^{s}-q_{\gamma}^{-s}\right) \cdot\right. \\
\left.\cdot \prod_{(r \delta, i),(s \delta, j) \in \widetilde{\Phi}_{+}^{\mathrm{im}}}\left(q_{i}^{r}-q_{i}^{-r}\right)^{f_{(r \delta, i)}}\left(q_{j}^{s}-q_{j}^{-s}\right)^{e_{(s \delta, j)}}\right) \cdot \mathfrak{U}_{0}^{M}
\end{gathered}
$$

for almost all the $\phi=\left(f_{\alpha}\right)_{\alpha \in \widetilde{\Phi}_{+}}, \eta=\left(e_{\alpha}\right)_{\alpha \in \widetilde{\Phi}_{+}} \in \mathbb{N}^{\widetilde{\Phi}_{+}}$.

$\underline{\text { Remark: }}$ indeed, what we do in Definition 5.8 is a "refinement" of the construction provided in [Dr], §7, to locate inside a quantized formal series Hopf algebra — whose semiclassical limit is a formal Poisson group $G^{\infty}$ - a quantized universal enveloping algebra - whose semiclassical limit yields the Lie bialgebra dual to that of $G^{\infty}$; see also [Ga3]. This will permit us to specialize at roots of 1 , which is not possible in [Dr].

Theorem 5.9. (a) $\mathfrak{U}^{M}(\hat{\mathfrak{h}})$ is a topological Hopf subalgebra (over $R$ ) of $U_{q}^{M}(\hat{\mathfrak{h}})$.

(b) $\mathfrak{U}^{M}(\hat{\mathfrak{h}})$ is an $R$-integer form of $U_{q}^{M}(\hat{\mathfrak{h}})$ and $\Omega_{M}$ (as topological Hopf algebras).

Proof. By construction $\mathfrak{U}^{M}(\hat{\mathfrak{h}})$ is an $R$-subalgebra of $U_{q}^{M}(\hat{\mathfrak{h}})$ and of $\Omega_{M}$; moreover, Theorem 5.2 and Proposition 4.9(b) ensure that $\Omega_{M}^{\vee}$ is an $R$-integer form (in topological sense) of $\Omega_{M}$ (as an algebra), hence also $\mathfrak{U}^{M}(\hat{\mathfrak{h}})$ is. Furthermore, Proposition $4.9(b)$ and Lemma 5.4 imply that $\Omega_{M}^{\vee}$ is a (topological) Hopf subalgebra of $\Omega_{M}$. Now the analysis in $\S 4.13$ via $\nu_{M}^{-1}$ gives $S\left(\mathfrak{U}^{M}(\hat{\mathfrak{h}})\right)=\mathfrak{U}^{M}(\hat{\mathfrak{h}})$ and $\Delta\left(\mathfrak{U}^{M}(\hat{\mathfrak{h}})\right) \subseteq \mathfrak{U}^{M}(\hat{\mathfrak{h}}) \widehat{\otimes} \mathfrak{U}^{M}(\hat{\mathfrak{h}})$, thus $\mathfrak{U}^{M}(\hat{\mathfrak{h}})$ is also a topological Hopf subalgebra. The claim follows.

$\underline{\text { Remark: }}$ in addition, the analysis in $\S 4.13$ shows - via $\nu_{M}{ }^{-1}$ - also that $\mathfrak{U}^{M}(\hat{\mathfrak{h}})$ contains the minimal topological Hopf subalgebra (over $R$ ) of $U_{q}^{M}(\hat{\mathfrak{h}})$ (or of $\Omega_{M}$ ) which contains $\hat{\mathfrak{H}}_{M}$ : this follows from formulas (4.7) and (4.9) and condition (5.3) (which is modeled on (4.7) indeed). In fact for our purposes we might also take this minimal algebra to play the role of $\mathfrak{U}^{M}(\hat{\mathfrak{h}})$ : the crux fact in any case is that (5.3) holds.

5.10 Presentation of $\mathfrak{U}^{M}(\hat{\mathfrak{h}})$. From the similar result available for $\mathfrak{U}^{M}(\hat{\mathfrak{g}})$ (cf. [DL], $\S 3.4$ : it deals with the finite case, but it is the like) we get a presentation of $\mathfrak{U}^{M}(\hat{\mathfrak{h}})$ by (topological) generators and relations. The algebra $\hat{\mathfrak{H}}_{M}$ of $\S 5.8$ is the associative $R$-algebra with 1 with generators

$$
F_{i}^{(s)}, \quad M_{j}, \quad M_{j}^{-1}, \quad\left(\begin{array}{c}
M_{j} ; c \\
t
\end{array}\right), \quad E_{i}^{(r)}
$$

$\left(i \in I ; j \in I_{\infty} ; c \in \mathbb{Z}, t, r, s \in \mathbb{N}\right.$; here we set $\left.M_{j}:=L_{\mu_{j}}\right)$, and relations

$$
M_{j} M_{j}^{-1}=1=M_{j}^{-1} M_{j}, \quad M_{j}^{ \pm 1} M_{j}^{ \pm 1}=M_{j}^{ \pm 1} M_{j}^{ \pm 1}
$$




$$
\begin{aligned}
& M_{j}^{ \pm 1}\left(\begin{array}{c}
M_{j} ; c \\
t
\end{array}\right)=\left(\begin{array}{c}
M_{j} ; c \\
t
\end{array}\right) M_{j}^{ \pm 1}, \quad\left(\begin{array}{c}
M_{j} ; c \\
0
\end{array}\right)=0, \quad\left(q_{i}-1\right)\left(\begin{array}{c}
M_{j} ; 0 \\
1
\end{array}\right)=M_{j}-1 \\
& \left(\begin{array}{c}
M_{j} ; c \\
t
\end{array}\right)\left(\begin{array}{c}
M_{j} ; c-t \\
s
\end{array}\right)=\left(\begin{array}{c}
t+s \\
t
\end{array}\right)_{q}\left(\begin{array}{c}
M_{j} ; c \\
t+s
\end{array}\right) \\
& \left(\begin{array}{c}
M_{j} ; c+1 \\
t
\end{array}\right)-q^{t}\left(\begin{array}{c}
M_{j} ; c \\
t
\end{array}\right)=\left(\begin{array}{c}
M_{j} ; c \\
t-1
\end{array}\right), \quad \forall t \geq 1 \\
& \left(\begin{array}{c}
M_{j} ; c \\
t
\end{array}\right)=\sum_{p \geq 0}^{p \leq c, t} q^{(c-p)(t-p)}\left(\begin{array}{c}
c \\
p
\end{array}\right)_{q}\left(\begin{array}{c}
M_{j} ; 0 \\
t-1
\end{array}\right), \quad \forall c \geq 0 \\
& \left(\begin{array}{c}
M_{j} ;-c \\
t
\end{array}\right)=\sum_{p=0}^{t}(-1)^{p} q^{-t(c+p)+p(p+1) / 2}\left(\begin{array}{c}
p+c-1 \\
p
\end{array}\right)_{q}\left(\begin{array}{c}
M_{j} ; 0 \\
t-p
\end{array}\right), \quad \forall c \geq 1 \\
& \left(\begin{array}{c}
M_{j} ; c+1 \\
t
\end{array}\right)-\left(\begin{array}{c}
M_{j} ; c \\
t
\end{array}\right)=q^{c-t+1} M_{j}\left(\begin{array}{c}
M_{j} ; c \\
t-1
\end{array}\right), \quad \forall t \geq 1 \\
& M_{j} E_{j}^{(p)}=q^{+p\left(\alpha_{j} \mid \mu_{i}\right)} E_{j}^{(p)} M_{j}, M_{j} F_{j}^{(p)}=q^{-p\left(\alpha_{j} \mid \mu_{i}\right)} F_{j}^{(p)} M_{j} \\
& \left(\begin{array}{c}
M_{j} ; c \\
t
\end{array}\right) E_{j}^{(p)}=E_{j}^{(p)}\left(\begin{array}{c}
M_{j} ; c+p\left(\alpha_{j} \mid \mu_{i}\right) \\
t
\end{array}\right) \\
& \left(\begin{array}{c}
M_{j} ; c \\
t
\end{array}\right) F_{j}^{(p)}=F_{j}^{(p)}\left(\begin{array}{c}
M_{j} ; c-p\left(\alpha_{j} \mid \mu_{i}\right) \\
t
\end{array}\right) \\
& E_{i}^{(r)} E_{i}^{(s)}=\left[\begin{array}{c}
r+s \\
r
\end{array}\right]_{q_{i}} E_{i}^{(r+s)}, \quad F_{i}^{(r)} F_{i}^{(s)}=\left[\begin{array}{c}
r+s \\
r
\end{array}\right]_{q_{i}} F_{i}^{(r+s)} \\
& \sum_{r+s=1-a_{i j}}(-1)^{s} E_{i}^{(r)} E_{j} E_{i}^{(s)}=0, \sum_{r+s=1-a_{i j}}(-1)^{s} F_{i}^{(r)} F_{j} F_{i}^{(s)}=0, \quad \forall i \neq j \\
& E_{i}^{(0)}=1, \quad E_{i}^{(r)} F_{j}^{(s)}=F_{j}^{(s)} E_{i}^{(r)}, \quad F_{i}^{(0)}=1
\end{aligned}
$$

Then $\mathfrak{U}^{M}(\hat{\mathfrak{h}})$ is the completion of $\hat{\mathfrak{H}}_{M}$ obtained by taking formal series in the PBW monomials of $\mathfrak{U}_{-}$and $\mathfrak{U}_{+}$, with coefficients in $\mathfrak{U}_{0}^{M}$, which satisfy the "growth condition" in Definition 5.8. Finally, formulas in $\S 4.13$ yield the following (with $K_{i}:=L_{\alpha_{i}}$ ):

$$
\begin{aligned}
& \Delta\left(F_{i}\right) \equiv F_{i} \otimes 1+1 \otimes F_{i}+\left(q_{i}-1\right) \cdot\left(\begin{array}{c}
K_{i} ; 0 \\
1
\end{array}\right) \otimes F_{i}+ \\
& +\left(q_{i}-q_{i}^{-1}\right)^{-1} \cdot \sum_{\substack{\alpha, \beta \in \widetilde{\Phi}_{+} \\
p(\alpha)-p(\beta)=-\alpha_{i}}} C_{\alpha, \beta}^{i,+}\left(q_{\alpha}-q_{\alpha}^{-1}\right)\left(q_{\beta}-q_{\beta}^{-1}\right) \cdot K_{i} E_{\alpha} \otimes F_{\beta} \quad \bmod \left(q-q^{-1}\right)^{2} \\
& \Delta\left(\left(\begin{array}{c}
L_{\mu_{i}} ; 0 \\
1
\end{array}\right)\right) \equiv\left(\begin{array}{c}
L_{\mu_{i}} ; 0 \\
1
\end{array}\right) \otimes 1+1 \otimes\left(\begin{array}{c}
L_{\mu_{i}} ; 0 \\
1
\end{array}\right)+\left(q_{i}-1\right) \cdot\left(\begin{array}{c}
L_{\mu_{i}} ; 0 \\
1
\end{array}\right) \otimes\left(\begin{array}{c}
L_{\mu_{i}} ; 0 \\
1
\end{array}\right)+ \\
& +(2)_{q^{-1}}^{2}\left(d_{i}\right)_{q}^{-1} \cdot \sum_{\substack{\alpha, \beta \in \widetilde{\Phi}_{+} \\
p(\alpha)-p(\beta)=0}}(q-1) C_{\alpha, \beta}\left[d_{\gamma}\right]_{q}\left[\left(\mu_{i} \mid \gamma\right)\right]_{q} \cdot L_{\mu_{i}} E_{\alpha} \otimes F_{\beta} L_{\mu_{i}} \bmod \left(q-q^{-1}\right)^{2}
\end{aligned}
$$




$$
\begin{array}{cc}
\Delta\left(E_{i}\right) \equiv 1 \otimes E_{i}+E_{i} \otimes 1+\left(q_{i}-1\right) \cdot E_{i} \otimes\left(\begin{array}{c}
K_{i} ; 0 \\
1
\end{array}\right)- & \\
-\left(q_{i}-q_{i}^{-1}\right)^{-1} \cdot \sum_{\substack{\alpha, \beta \in \widetilde{\Phi}_{+} \\
p(\alpha)-p(\beta)=+\alpha_{i}}} C_{\alpha, \beta}^{i,-}\left(q_{\alpha}-q_{\alpha}^{-1}\right)\left(q_{\beta}-q_{\beta}^{-1}\right) E_{\alpha} \otimes F_{\beta} K_{i} & \bmod \left(q-q^{-1}\right)^{2} \\
S\left(F_{i}\right) \equiv-q_{i}^{-2} \cdot F_{i} K_{i}^{-1}, \quad S\left(E_{i}\right) \equiv-q_{i}^{+2} \cdot K_{i}^{-1} E_{i} & \bmod \left(q-q^{-1}\right) \\
S\left(\left(\begin{array}{c}
M_{j} ; 0 \\
1
\end{array}\right)\right) \equiv-M_{j}^{-1} \cdot\left(\begin{array}{c}
M_{j} ; 0 \\
1
\end{array}\right) & \bmod \left(q-q^{-1}\right) \\
\epsilon\left(F_{i}\right)=0, \quad \epsilon\left(\left(\begin{array}{c}
M_{j} ; 0 \\
1
\end{array}\right)\right)=0, \quad \epsilon\left(E_{i}\right)=0 . &
\end{array}
$$

5.11 Quantum Poisson pairing. Since $j_{M}{ }^{-1} \circ \nu_{M}: U_{q}^{M}(\hat{\mathfrak{h}}) \stackrel{\cong}{\longrightarrow} U_{q}^{M^{\prime}}(\hat{\mathfrak{g}})^{*}$, via evaluation we get a perfect Hopf pairing

$$
\pi_{q}^{M}: U_{q}^{M}(\hat{\mathfrak{h}}) \otimes U_{q}^{M^{\prime}}(\hat{\mathfrak{g}}) \longrightarrow \mathbb{C}(q)
$$

defined by $\pi_{q}^{M}(h, g):=\left\langle j_{M}{ }^{-1}\left(\nu_{M}(h)\right), g\right\rangle$ for all $h \in U_{q}^{M}(\hat{\mathfrak{h}}), g \in U_{q}^{M^{\prime}}(\hat{\mathfrak{g}})$.

We call $\pi_{q}^{M}$ quantum Poisson pairing.

By previous analysis, the integer forms of quantum enveloping algebras are $R$-dual of each other (cf. $\S 2.7$ ) with respect to $\pi_{q}^{M}$; so the latter restrict to perfect Hopf pairings

$$
\pi_{q, \widehat{H}_{M}}: \mathfrak{U}^{M^{\prime}}(\hat{\mathfrak{h}}) \otimes \mathcal{U}^{M}(\hat{\mathfrak{g}}) \longrightarrow R, \quad \pi_{q, \widehat{G}_{M}^{\infty}}: \mathcal{U}^{M}(\hat{\mathfrak{h}}) \otimes \mathfrak{U}^{M^{\prime}}(\hat{\mathfrak{g}}) \longrightarrow R
$$

\section{$\S 6$ Specialization at roots of 1}

6.1 The case $q \rightarrow 1$ : specialization of $\mathfrak{U}^{M}(\hat{\mathfrak{h}})$ to $U(\hat{\mathfrak{h}})$ and consequences. To begin with, set

$$
\mathfrak{U}_{1}^{M}(\hat{\mathfrak{h}}):=\mathfrak{U}^{M}(\hat{\mathfrak{h}}) /(q-1) \mathfrak{U}^{M}(\hat{\mathfrak{h}}) \cong \mathfrak{U}^{M}(\hat{\mathfrak{h}}) \otimes_{R} \mathbb{C}
$$

and let $p_{1}: \mathfrak{U}^{M}(\hat{\mathfrak{h}}) \rightarrow \mathfrak{U}_{1}^{M}(\hat{\mathfrak{h}})$ be the canonical projection; then set $\mathrm{f}_{i}:=p_{1}\left(F_{i}^{(1)}\right)$, $\mathrm{m}_{i}:=p_{1}\left(\left(\begin{array}{c}M_{j} ; 0 \\ 1\end{array}\right)\right), \mathrm{e}_{i}:=p_{1}\left(E_{i}^{(1)}\right)$, (where $\left.M_{j}:=L_{\mu_{j}}\right)$ for all $i \in I, j \in I_{\infty}$.

Theorem 6.2. For $q \rightarrow 1, \mathfrak{U}^{M}(\hat{\mathfrak{h}})$ specializes to the Poisson Hopf coalgebra $U(\hat{\mathfrak{h}})$; in other words, there exists an isomorphism of Poisson Hopf coalgebras

$$
\mathfrak{U}_{1}^{M}(\hat{\mathfrak{h}}) \cong U(\hat{\mathfrak{h}}) .
$$

Proof. The proof mimick that for $\mathfrak{U}_{1}^{M}(\hat{\mathfrak{g}}) \cong U(\hat{\mathfrak{g}})$. From the very definition of $\mathfrak{U}^{M}(\hat{\mathfrak{h}})$ we get $\mathfrak{U}_{1}^{M}(\hat{\mathfrak{h}})=\left.\hat{\mathfrak{H}}_{M}\right|_{q=1}:=\hat{\mathfrak{H}}_{M} /(q-1) \hat{\mathfrak{H}}_{M}$, hence we are reduced to study $\left.\hat{\mathfrak{H}}_{M}\right|_{q=1} ;$ 
moreover the presentation of $\hat{\mathfrak{H}}_{M}$ provides one of $\left.\hat{\mathfrak{H}}_{M}\right|_{q=1}$. Now the definition of $\left.\hat{\mathfrak{H}}_{M}\right|_{q=1}$ and the explicit form of the PBW basis of $\mathfrak{U}_{0}^{M}$ (cf. §2.6) imply that the elements $F_{i}^{(r)},\left(\begin{array}{c}M_{j} ; 0 \\ t\end{array}\right), M_{j}^{-1}, E_{i}^{(s)}\left(i \in I, j \in I_{\infty} ; r, t, s \in \mathbb{N}\right)$ are enough to generate $\hat{\mathfrak{H}}_{M} ;$ finally, straightforward computation gives $p_{1}\left(F_{i}^{(r)}\right)=\frac{\mathrm{f}_{i}^{r}}{r !}, \quad p_{1}\left(\left(\begin{array}{c}M_{j} ; 0 \\ t\end{array}\right)\right)=\left(\begin{array}{c}\mathrm{m}_{j} \\ t\end{array}\right)$, $p_{1}\left(M_{j}^{-1}\right)=1, \quad p_{1}\left(E_{i}^{(s)}\right)=\frac{\mathrm{e}_{i}^{s}}{s !} \quad\left(\right.$ where $\left.\left(\begin{array}{c}\mathrm{m}_{i} \\ t\end{array}\right):=\frac{\mathrm{m}_{i}\left(\mathrm{~m}_{i}-1\right)\left(\mathrm{m}_{i}-2\right) \cdots\left(\mathrm{m}_{i}-t+1\right)}{t !}\right)$, hence $\mathfrak{U}_{1}^{M}(\hat{\mathfrak{h}})=\left.\hat{\mathfrak{H}}_{M}\right|_{q=1}$ is generated by the $f_{i}$ 's, the $\mathrm{m}_{i}$ 's, and the $\mathrm{e}_{i}$ 's, with some relations.

When $M=Q_{\infty}$ this presentation is exactly the same of $U(\hat{\mathfrak{h}})$ (cf. (1.1)), with $\mathrm{h}_{i}=\mathrm{m}_{i}$; in addition, comparing (1.2) with formulas in $\S 5.10$ (for $q=1$ ) shows that also the Hopf structure is the same. In particular $\mathfrak{U}_{1}^{Q \infty}(\hat{\mathfrak{h}})$ is cocommutative, hence has a canonical co-Poisson structure, given by $\delta:=\frac{\Delta-\Delta^{o p}}{q-1}$, described by formulas — deduced from those in $\$ 5.10$ - which do coincide with (1.3), as a straightforward checking shows.

Finally, for $M \neq Q_{\infty}$ we prove that $\mathfrak{U}_{1}^{M}(\hat{\mathfrak{h}}) \cong \mathfrak{U}_{1}^{Q \infty}(\hat{\mathfrak{h}})$ as Poisson Hopf coalgebras: since $\mathfrak{U}^{M}(\hat{\mathfrak{h}}) \supseteq \mathfrak{U}^{Q_{\infty}}(\hat{\mathfrak{h}})$ by definition, it is enough to check that $\left.\hat{\mathfrak{H}}_{M}\right|_{q=1}=\left.\hat{\mathfrak{H}}_{Q_{\infty}}\right|_{q=1}$ as $\mathbb{C}$-vector spaces. Assume we are in the simply laced case. Since $M_{i}:=L_{\mu_{i}}$ and $K_{j}:=L_{\alpha_{j}}$, it is $K_{j}:=\prod_{i=1}^{n} M_{i}^{c_{i j}}$, where $c_{i j} \in \mathbb{Z}$ are such that $\alpha_{j}=\sum_{i=1}^{n} c_{i j} \mu_{i}$. Then $\left.\left(\begin{array}{c}K_{j} ; 0 \\ t\end{array}\right)\right|_{q=1}=\left.\sum_{i=1}^{n} c_{i j} \cdot\left(\begin{array}{c}M_{i} ; 0 \\ t\end{array}\right)\right|_{q=1}$ so that $\left.\mathfrak{U}_{0}^{M}\right|_{q=1}=\left.\mathfrak{U}_{0}^{Q \infty}\right|_{q=1}$ follows, whence $\left.\hat{\mathfrak{H}}_{M}\right|_{q=1}=\left.\hat{\mathfrak{H}}_{Q_{\infty}}\right|_{q=1}$, q.e.d. In the other cases $M=P_{\infty}$, and this argument still works, mutatis mutandis, because $\alpha_{j}=\sum_{i=1}^{n} a_{i j} \omega_{i}$, hence $K_{j}:=\prod_{i=1}^{n} L_{\alpha_{i}}^{a_{i j}}$, so that $\left.\left(\begin{array}{c}K_{j} ; 0 \\ t\end{array}\right)\right|_{q=1}=\left.\sum_{i=1}^{n} a_{j i} \cdot\left(\begin{array}{c}L_{i} ; 0 \\ t\end{array}\right)\right|_{q=1}$ and we are done again.

Theorem 6.2 has an interesting corollary, namely $\mathcal{U}^{M}(\hat{\mathfrak{g}}) \stackrel{q \rightarrow 1}{\longrightarrow} F\left[\widehat{H}_{M}\right]$ (cf. $\left.\S 3.4\right)$. The original proof in $[\mathrm{BK}]$ is lenghty involved, and requires hard computations; on the contrary, we can deduce this result as an easy consequence of the previous one:

Theorem 6.3. The Hopf algebra $\mathcal{U}^{M}(\hat{\mathfrak{g}})$ specializes to the Poisson Hopf algebra $F\left[\widehat{H}_{M}\right]$ for $q \rightarrow 1$, in other words, there exists an isomorphism of Poisson Hopf algebras

$$
\mathcal{U}_{1}^{M}(\hat{\mathfrak{g}}):=\mathcal{U}^{M}(\hat{\mathfrak{g}}) /(q-1) \mathcal{U}^{M}(\hat{\mathfrak{g}}) \cong F\left[\widehat{H}_{M}\right]
$$

Proof. Since $\mathcal{U}^{M}(\hat{\mathfrak{g}})$ is perfectly paired with $\mathfrak{U}^{M^{\prime}}(\hat{\mathfrak{h}})$, we have that $\mathcal{U}_{1}^{M}(\hat{\mathfrak{g}})$ is perfectly paired with $\mathfrak{U}_{1}^{M^{\prime}}(\hat{\mathfrak{h}}) \cong U(\hat{\mathfrak{h}})$ : the latter is cocommutative, hence the former is commutative. Then $\mathcal{U}_{1}^{M}(\hat{\mathfrak{g}})$ is a commutative Hopf algebra over $\mathbb{C}$, with countably many generators: hence it is the algebra of regular functions of a complex affine proalgebraic group, say $\widehat{H}^{\prime}$; moreover $\mathcal{U}_{1}^{M}(\hat{\mathfrak{g}})=F\left[\widehat{H}^{\prime}\right]$ inherits from $\mathcal{U}^{M}(\hat{\mathfrak{g}})$ a Poisson structure, so $\widehat{H}^{\prime}$ is a Poisson (proalgebraic) group. It is clear from the presentation of $\mathcal{U}^{M}(\hat{\mathfrak{g}})$ 
that $F\left[\widehat{H}^{\prime}\right]\left(=\mathcal{U}_{1}^{M}(\hat{\mathfrak{g}})\right) \cong F\left[\widehat{H}_{M}\right]$ as Hopf algebras, hence $\widehat{H}^{\prime}=\widehat{H}_{M}$ as proalgebraic groups (the non-trivial part, as in $[\mathrm{BK}]$, is about the Poisson structure!). Now the Hopf pairing among $\mathfrak{U}_{1}^{M^{\prime}}(\hat{\mathfrak{h}}) \cong U(\hat{\mathfrak{h}})$ and $\mathcal{U}_{1}^{M}(\hat{\mathfrak{g}})=F\left[\widehat{H}^{\prime}\right]=F\left[\widehat{H}_{M}\right]$ is compatible with Poisson and co-Poisson structures, that is $\langle h,\{f, g\}\rangle=\langle\delta(h), f \otimes g\rangle$, where $\delta$ is the Poisson cobracket of $\mathfrak{U}_{1}^{M^{\prime}}(\hat{\mathfrak{h}})=U(\hat{\mathfrak{h}})$ and $\{$,$\} is either the Poisson bracket \{,\}_{\star}$ of $\widehat{H}_{M}$ or the Poisson bracket $\{,\}_{\circ}$ of $\widehat{H}^{\prime}$ : since the pairing is perfect, we must have $\{,\}_{\star}=\{,\}_{\circ}$, q.e.d.

6.4 The case $q \rightarrow 1$ : specialization of $\mathcal{U}^{M}(\hat{\mathfrak{h}})$ to $F^{\infty}\left[\widehat{G}_{M}\right]$. We are going to show that $\mathcal{U}^{M}(\hat{\mathfrak{h}})$ is a quantization of $F^{\infty}\left[\widehat{G}_{M}\right]$; such a result can be seen as (Poisson) dual counterpart of $\mathcal{U}^{M}(\hat{\mathfrak{g}}) \stackrel{q \rightarrow 1}{\longrightarrow} F\left[\widehat{H}_{M}\right]$ (cf. Theorem 6.3). As usual, we set

$$
\mathcal{U}_{1}^{M}(\hat{\mathfrak{h}}):=\mathcal{U}^{M}(\hat{\mathfrak{h}}) /(q-1) \mathcal{U}^{M}(\hat{\mathfrak{h}}) \cong \mathcal{U}^{M}(\hat{\mathfrak{h}}) \otimes_{R} \mathbb{C}
$$

Theorem 6.5. The formal Hopf algebra $\mathcal{U}^{M}(\hat{\mathfrak{h}})$ specializes to the formal Poisson Hopf algebra $F^{\infty}\left[\widehat{G}_{M}\right]$ for $q \rightarrow 1$; in other words, there exists an isomorphism of formal Poisson Hopf algebras

$$
\mathcal{U}_{1}^{M}(\hat{\mathfrak{h}}) \cong F^{\infty}\left[\widehat{G}_{M}\right]
$$

Proof. Recall that $F^{\infty}\left[\widehat{G}_{M}\right]$ is isomorphic to the linear dual of $U(\hat{\mathfrak{g}})$, that is $F^{\infty}\left[\widehat{G}_{M}\right] \cong$ $U(\hat{\mathfrak{g}})^{*}$. On the other hand, we have an isomorphism $j_{M}{ }^{-1} \circ \nu_{M}: U_{q}^{M}(\hat{\mathfrak{h}}) \stackrel{\cong}{\longrightarrow} U_{q}^{M^{\prime}}(\hat{\mathfrak{g}})^{*}$ of formal Hopf algebras, and Theorem 5.6 ensures that it restricts to

$$
j_{M}{ }^{-1} \circ \nu_{M}: \mathcal{U}^{M}(\hat{\mathfrak{h}}) \stackrel{\cong}{\longrightarrow} \mathfrak{U}^{M^{\prime}}(\hat{\mathfrak{g}})^{*} .
$$

When $q \longrightarrow 1$, we have that $\mathfrak{U}^{M^{\prime}}(\hat{\mathfrak{g}})$ specializes to $U(\hat{\mathfrak{g}})$, therefore (6.1) implies $\mathcal{U}_{1}^{M}(\hat{\mathfrak{h}}) \cong \mathfrak{U}^{M^{\prime}}(\hat{\mathfrak{g}})^{*} \otimes_{R} \mathbb{C}=\mathfrak{U}_{1}^{M^{\prime}}(\hat{\mathfrak{g}})^{*} \cong U(\hat{\mathfrak{g}})^{*}=F^{\infty}\left[\widehat{G}_{M}\right]=F^{\infty}\left[\widehat{G}_{M}\right]$, q.e.d.

6.6 The case $q \rightarrow \varepsilon$ : quantum Frobenius morphisms. Let $\varepsilon$ be a primitive $\ell$-th root of 1 in $k$, with $\ell$ odd satisfying (3.8) and such that $\ell>d:=\max _{i}\left\{d_{i}\right\}$, and set

$$
\mathfrak{U}_{\varepsilon}^{M}(\hat{\mathfrak{h}}):=\mathfrak{U}^{M}(\hat{\mathfrak{h}}) /(q-\varepsilon) \mathfrak{U}^{M}(\hat{\mathfrak{h}}) \cong \mathfrak{U}^{M}(\hat{\mathfrak{h}}) \otimes_{R} \mathbb{C}
$$

The next result is the analogue of (3.5) for $U_{q}^{M}(\hat{\mathfrak{h}})$.

Theorem 6.7. There exists a continuous epimorphism of formal Hopf algebras

$$
\mathfrak{F r}_{\hat{\mathfrak{h}}}: \mathfrak{U}_{\varepsilon}^{M}(\hat{\mathfrak{h}}) \longrightarrow \mathfrak{U}_{1}^{M}(\hat{\mathfrak{h}}) \cong U(\hat{\mathfrak{h}})
$$


defined by (for all $i \in I, j \in I_{\infty}$ )

$\mathfrak{F}_{\mathfrak{h}}:\left\{\begin{array}{l}\left.\left.F_{i}{ }^{(s)}\right|_{q=\varepsilon} \mapsto F_{i}^{(s / \ell)}\right|_{q=1},\left.\left.\left(\begin{array}{c}M_{j} ; 0 \\ s\end{array}\right)\right|_{q=\varepsilon} \mapsto\left(\begin{array}{c}M_{j} ; 0 \\ s / \ell\end{array}\right)\right|_{q=1},\left.\left.E_{i}^{(s)}\right|_{q=\varepsilon} \mapsto E_{i}^{(s / \ell)}\right|_{q=1} \text { if } \ell \mid s \\ \left.F_{i}^{(s)}\right|_{q=\varepsilon} \mapsto 0,\left.\quad\left(\begin{array}{c}M_{j} ; 0 \\ s\end{array}\right)\right|_{q=\varepsilon} \mapsto 0,\left.\quad E_{i}^{(s)}\right|_{q=\varepsilon} \mapsto 0 \quad \text { otherwise } \\ \left.M_{i}^{-1}\right|_{q=1} \mapsto 1\end{array}\right.$

which is adjoint of $\mathcal{F} r_{\hat{\mathfrak{g}}}$ (cf. (3.9)) with respect to the quantum Poisson pairings, i.e.

$$
\pi_{1, H_{M^{\prime}}}\left(\mathfrak{F r}_{\hat{\mathfrak{h}}}(h), g\right)=\pi_{\varepsilon, H_{M^{\prime}}}\left(h, \mathcal{F} r_{\hat{\mathfrak{g}}}(g)\right) \quad \forall h \in \mathfrak{U}_{\varepsilon}^{M}(\hat{\mathfrak{h}}), g \in \mathcal{U}_{\varepsilon}^{M^{\prime}}(\hat{\mathfrak{g}}) .
$$

Proof. The formulas above uniquely determine a continuous Hopf algebra epimorphism $\mathfrak{F}_{\hat{\mathfrak{h}}}$, if any, because $\left.F_{i}^{(s)}\right|_{q=\varepsilon},\left.\left(\begin{array}{c}M_{j} ; 0 \\ s\end{array}\right)\right|_{q=\varepsilon},\left.M_{j}^{-1}\right|_{q=\varepsilon},\left.E_{i}^{(s)}\right|_{q=\varepsilon}$ are topological generators of the algebra $\mathfrak{U}_{\varepsilon}^{M}(\hat{\mathfrak{h}})$. Now consider the embedding $\mathcal{F} r_{\hat{\mathfrak{g}}}: F\left[\widehat{H}_{M^{\prime}}\right] \cong$ $\mathcal{U}_{1}^{M^{\prime}}(\hat{\mathfrak{g}}) \hookrightarrow \mathcal{U}_{\varepsilon}^{M^{\prime}}(\hat{\mathfrak{g}})$ of Hopf algebras (cf. (3.9)): its dual is a (continuous) epimorphism of formal Hopf algebras $\mathcal{U}_{\varepsilon}^{M^{\prime}}(\hat{\mathfrak{g}})^{*} \longrightarrow \mathcal{U}_{1}^{M^{\prime}}(\hat{\mathfrak{g}})^{*}$. On the other hand we have an embedding $\mathfrak{U}_{\varepsilon}^{M}(\hat{\mathfrak{h}}) \hookrightarrow \mathcal{U}_{\varepsilon}^{M^{\prime}}(\hat{\mathfrak{g}})^{*}$ provided by the specialized quantum Poisson pairing $\pi_{\varepsilon, H_{M^{\prime}}}: \mathfrak{U}_{\varepsilon}^{M}(\hat{\mathfrak{h}}) \otimes \mathcal{U}_{\varepsilon}^{M^{\prime}}(\hat{\mathfrak{g}}) \longrightarrow \mathbb{C}:$ therefore composition yields a morphism $\mathfrak{F r}_{\hat{\mathfrak{h}}}: \mathfrak{U}_{\varepsilon}^{M}(\hat{\mathfrak{h}}) \longrightarrow \mathcal{U}_{1}^{M^{\prime}}(\hat{\mathfrak{g}})^{*}$. Furthermore, the very construction gives $\left\langle\mathfrak{F r}_{\hat{\mathfrak{h}}}(h), g\right\rangle=$ $\pi_{1, H_{M^{\prime}}}\left(\mathfrak{F r}_{\hat{\mathfrak{h}}}(h), g\right)=\pi_{\varepsilon, H_{M^{\prime}}}\left(h, \mathcal{F} r_{\hat{\mathfrak{g}}}(g)\right)$, hence $\mathfrak{F r}_{\hat{\mathfrak{h}}}$ is adjoint of $\mathcal{F} r_{\hat{\mathfrak{g}}}(g)$, and is described by the formulas above. Finally, we have to prove that $\mathfrak{F}_{\mathfrak{h}}$ has image $\mathfrak{U}_{1}^{M}(\hat{\mathfrak{h}})$.

The problem is that $\mathfrak{U}_{\varepsilon}^{M}(\hat{\mathfrak{h}})$ is made of series, say infinite linear combinations of PBW monomials - whereas $\mathfrak{U}_{1}^{M}(\hat{\mathfrak{h}})$ is made only of finite linear combinations of (the same type of) PBW monomials: so we must show that these infinite linear combinations indeed are mapped by $\mathfrak{F}_{\mathfrak{\mathfrak { h }}}$ to finite ones.

Recall the definition of $\mathfrak{U}^{M}(\hat{\mathfrak{h}})$ (see Definition 5.8(b)), in particular condition (5.3): when $q=\varepsilon$ (a root of one of odd order $\ell$ satisfying (3.8)) the right-hand-side in (5.3) vanishes as soon as $\varphi$ and $\eta$ are such that $f_{\beta} \geq \ell$ or $e_{\gamma} \geq \ell$ for some $\beta, \gamma \in \Phi_{+}^{\text {re }}$ (for in this case one of the factors in the upper line is zero); and, of course, almost all the PBW monomials $\mathfrak{F}_{\varphi}$ and $\mathfrak{E}_{\eta}$ which appear in the summands $\mathfrak{F}_{\varphi} \cdot \phi_{\varphi, \eta} \cdot \mathfrak{E}_{\eta}$ of the series expansion of an element of $\mathfrak{U}_{\varepsilon}^{M}(\hat{\mathfrak{h}})$ do satisfy one of (or both) the conditions $f_{\beta} \geq \ell, e_{\gamma} \geq \ell$ for all $\beta, \gamma \in \Phi_{+}^{\text {re }}$. Now let $(r \delta, i) \in \widetilde{\Phi}_{+}^{\text {im }}$; let $\dot{\bar{F}}_{(r \delta, i)}$ be the element of $\mathcal{U}_{-}$which is dual (with respect to $\pi$ or to $\bar{\pi}$ ) of $\frac{r}{[r]_{q_{i}}} E_{(r \delta, i)}$ : by Claim (a) in $\S 2.7$ we know that $\dot{\bar{F}}_{(r \delta, i)}$ is an $R$-linear combination of the $\bar{F}_{(r \delta, j)}$ 's, $j \in I_{0}$. Now, by (3.11) and the Claim in $\S 2.3$ we have that $\mathcal{F} r_{\hat{\mathfrak{g}}}$ maps $\dot{\bar{F}}_{(r \delta, i)}$ to $\ell \dot{\bar{F}}_{(r \ell \delta, i)}$ : the dual of the latter in $\mathfrak{U}_{\varepsilon}^{M}(\hat{\mathfrak{h}})$ is $\ell^{-1} \widehat{E}_{(r \ell \delta, i)}=\ell^{-1} \frac{r \ell}{[r \ell]_{q_{i}}} E_{(r \ell \delta, i)}$. Therefore $\mathcal{F} r_{\hat{\mathfrak{g}}}$ maps 
a PBW monomial in the $\bar{F}_{\gamma}$ 's $\left(\gamma \in \Phi_{+}^{\mathrm{re}}\right)$ and the $\dot{\bar{F}}_{(r \delta, i)}\left((r \delta, i) \in \widetilde{\Phi}_{+}^{\mathrm{im}}\right)$, call it $\dot{\mathcal{F}}_{\varphi}$, to a similar PBW monomial $\dot{\mathcal{F}}_{\varphi^{\prime}}$ such that $\varphi^{\prime}=\left(f_{\alpha}\right)_{\alpha \in \widetilde{\Phi}_{+}}$with $f_{(r \delta, i)}=0$ for all $i \in I_{0}$ and $r \in \mathbb{N} \backslash \ell \mathbb{N}$. Similarly occurs when reverting the roles of the $F$ 's and the $E$ 's. This fact clearly implies that, given a PBW monomial $g$ of $\mathcal{U}_{1}^{M^{\prime}}(\hat{\mathfrak{g}})$, we have $\left\langle\mathfrak{F r}_{\hat{\mathfrak{h}}}(h), g\right\rangle=\pi_{1, H_{M^{\prime}}}\left(\mathfrak{F r}_{\hat{\mathfrak{h}}}(h), g\right)=\pi_{\varepsilon, H_{M^{\prime}}}\left(h, \mathcal{F} r_{\mathfrak{\mathfrak { g }}}(g)\right)=0$ for all PBW monomials $h=\mathfrak{F}_{\varphi} \cdot u_{\tau} \cdot \mathfrak{E}_{\eta}$ of $\left.\hat{\mathfrak{H}}_{M}\right|_{q=\varepsilon}\left(\subset \mathfrak{U}_{\varepsilon}^{M}(\hat{\mathfrak{h}})\right)$ such that $f_{(r \delta, i)} \neq 0$ or $e_{(r \delta, i)} \neq 0$ for some $i \in I_{0}$ and $r \in \mathbb{N} \backslash \ell \mathbb{N}$ : thus $\mathfrak{F}_{\hat{\mathfrak{h}}}$ maps to zero this kind of PBW monomials of $\mathfrak{U}_{\varepsilon}^{M}(\hat{\mathfrak{h}})$. As for the other ones, we can read the condition $f_{(r \delta, i)}=0$ or $e_{(r \delta, i)}=0$ for all $i \in I_{0}$ and $r \in \mathbb{N} \backslash \ell \mathbb{N}$ as

$$
f_{(r \delta, i)} \neq 0 \Longrightarrow \ell\left|r, \quad e_{(r \delta, i)} \neq 0 \Longrightarrow \ell\right| r
$$

then in (5.3) the right-hand-side vanishes again (this time because, in this case, one of the factors in the lower line is zero). So the upshot is that $\mathfrak{F r}_{\mathfrak{h}}$ kills almost all the summands in the series expansion of any element of $\mathfrak{U}_{\varepsilon}^{M}(\hat{\mathfrak{h}})$, which solves our problem.

Corollary 6.8. The images of imaginary root vectors under the quantum Frobenius morphisms $\mathfrak{F r}_{\hat{\mathfrak{h}}}: \mathfrak{U}_{\varepsilon}^{M}(\hat{\mathfrak{h}}) \longrightarrow \mathfrak{U}_{1}^{M}(\hat{\mathfrak{h}})$ and $\mathfrak{F r}_{\hat{\mathfrak{g}}}: \mathfrak{U}_{\varepsilon}^{M}(\hat{\mathfrak{g}}) \longrightarrow \mathfrak{U}_{1}^{M}(\hat{\mathfrak{g}})$ are given by $\left(\right.$ for all $\left.(r \delta, i) \in \widetilde{\Phi}_{+}^{\mathrm{im}}\right)$

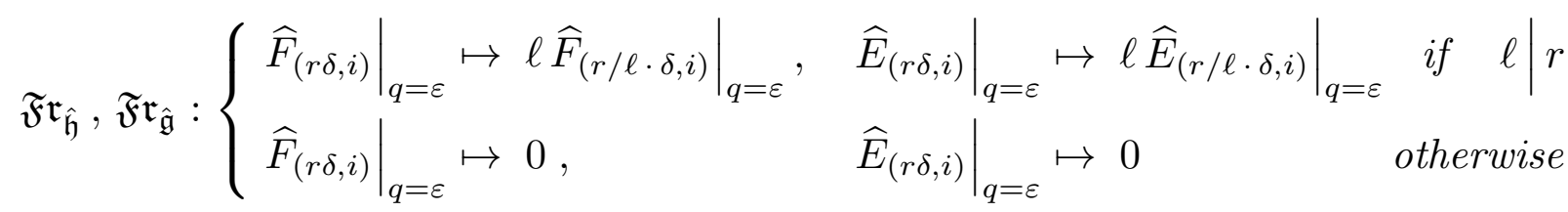

Proof. For $\mathfrak{F}_{\mathfrak{h}}$ the result follows from (3.11) by duality (cf. the proof of Theorem 6.7); since on quantum unipotent subalgebras $\mathfrak{F r}_{\hat{\mathfrak{g}}}$ coincides with $\mathfrak{F}_{\mathfrak{\mathfrak { h }}}$ (they are defined by the same formulas) the result follows for $\mathfrak{F}_{\mathfrak{\mathfrak { g }}}$ too (otherwise, we can get it again by duality from (6.4) below, with the same argument used for $\left.\mathfrak{F r}_{\mathfrak{h}}\right)$.

Similar arguments to those used to prove Theorem 6.7 provide a proof of the next result, which is the analogue of (3.9); as usual, we set

$$
\mathcal{U}_{\varepsilon}^{M}(\hat{\mathfrak{h}}):=\mathcal{U}^{M}(\hat{\mathfrak{h}}) /(q-\varepsilon) \mathcal{U}^{M}(\hat{\mathfrak{h}}) \cong \mathcal{U}^{M}(\hat{\mathfrak{h}}) \otimes_{R} \mathbb{C}
$$

moreover we define the set

$$
\mathbb{N}^{\widetilde{\Phi}_{+}}[\ell]:=\left\{\chi=\left(x_{\alpha}\right)_{\alpha \in \widetilde{\Phi}_{+}} \in \mathbb{N}^{\widetilde{\Phi}_{+}} \mid x_{\gamma} \in \ell \mathbb{N} \forall \gamma \in \Phi_{+}^{\mathrm{re}}, x_{(r \delta, i)}=0 \quad \forall i \in I_{0}, r \in \mathbb{N} \backslash \ell \mathbb{N}\right\}
$$


Theorem 6.9. (a) There exists a unique continuous monomorphism of formal Hopf algebras

$$
\mathcal{F} r_{\hat{\mathfrak{h}}}: F^{\infty}\left[\widehat{G}_{M}\right] \cong \mathcal{U}_{1}^{M}(\hat{\mathfrak{h}}) \longleftrightarrow \mathcal{U}_{\varepsilon}^{M}(\hat{\mathfrak{h}})
$$

defined (for all $\alpha \in \Phi_{+}^{\mathrm{re}}, \mu \in M$ ) by

$$
\mathcal{F} r_{\hat{\mathfrak{h}}}:\left.\left.\quad \bar{F}_{\alpha}\right|_{q=1} \mapsto \bar{F}_{\alpha}^{\ell}\right|_{q=\varepsilon},\left.\left.\quad L_{\mu}\right|_{q=1} \mapsto L_{\mu}^{\ell}\right|_{q=\varepsilon},\left.\left.\quad \bar{E}_{\alpha}\right|_{q=1} \mapsto \bar{E}_{\alpha}^{\ell}\right|_{q=\varepsilon}
$$

and enjoying

$$
\mathcal{F} r_{\hat{\mathfrak{g}}}:\left.\left.\bar{F}_{(r \delta, i)}\right|_{q=1} \mapsto \ell \bar{F}_{(r \ell \delta, i)}\right|_{q=\varepsilon},\left.\left.\bar{E}_{(r \delta, i)}\right|_{q=1} \mapsto \ell \bar{E}_{(r \ell \delta, i)}\right|_{q=\varepsilon} \quad \forall(r \delta, i) \in \widetilde{\Phi}_{+}^{\mathrm{im}}
$$

which is adjoint of $\mathfrak{F r}_{\mathfrak{g}}$ (cf. (3.5)) with respect to quantum Poisson pairings, that is

$$
\pi_{\varepsilon, \widehat{G}_{M}}\left(\mathcal{F} r_{\hat{\mathfrak{h}}}(h), g\right)=\pi_{1, \widehat{G}_{M}}\left(h, \mathfrak{F}_{\mathfrak{\mathfrak { g }}}(g)\right) \quad \forall h \in \mathcal{U}_{1}^{M}(\hat{\mathfrak{h}}), g \in \mathfrak{U}_{\varepsilon}^{M^{\prime}}(\hat{\mathfrak{g}}) .
$$

(b) The image $Z_{0}\left(\cong{ }_{\mathcal{F}} r_{\hat{\mathfrak{h}}} \mathcal{U}_{1}^{M}(\hat{\mathfrak{h}})\right)$ of $\mathcal{F} r_{\hat{\mathfrak{h}}}$ is a formal Hopf subalgebra contained in the centre of $\mathcal{U}_{\varepsilon}^{M}(\hat{\mathfrak{h}})$.

(c) The set $\left\{\mathcal{F}_{\phi} \cdot B_{\tau} \cdot \mathcal{E}_{\eta} \mid \phi, \eta \in \mathbb{N}^{\widetilde{\Phi}_{+}}[\ell], \tau \in \ell \mathbb{N}^{I_{\infty}}\right\}$ is a pseudobasis of $Z_{0}$ over $\mathbb{C}$.

(d) The set $\left\{\mathcal{F}_{\phi} \cdot B_{\tau} \cdot \mathcal{E}_{\eta} \mid \phi, \eta \in \mathbb{N}^{\widetilde{\Phi}_{+}} \backslash \mathbb{N}^{\widetilde{\Phi}_{+}}[\ell], \tau \in \mathbb{N} \backslash \ell \mathbb{N}^{I_{\infty}}\right\}$ is a pseudobasis of $\mathcal{U}_{\varepsilon}^{M}(\hat{\mathfrak{h}})$ over $Z_{0}$; therefore also the set of ordered PBW monomials $\left\{\mathcal{F}_{\phi} \cdot L_{\mu} \cdot \mathcal{E}_{\eta} \mid \phi, \eta \in\right.$ $\left.\mathbb{N}^{\widetilde{\Phi}_{+}} \backslash \mathbb{N}^{\widetilde{\Phi}_{+}}[\ell], \mu \in \mathbb{N} \backslash \ell \mathbb{N}^{I_{\infty}}\right\}$ is a $Z_{0}$-basis of $\mathcal{U}_{\varepsilon}^{M}(\hat{\mathfrak{h}})$, so $\mathcal{U}_{\varepsilon}^{M}(\hat{\mathfrak{h}})$ is a free $Z_{0}$-module.

Proof. Like in [BK], Lemma 3.2.2, we exploit the fact that $\left.\bar{F}_{\alpha}\right|_{q=1},\left.L_{\mu}\right|_{q=1},\left.\bar{E}_{\alpha}\right|_{q=1}$ $\left(\alpha \in \Phi_{+}^{\mathrm{re}}, \mu \in M\right)$ are generators of $\mathcal{U}_{1}^{M}(\hat{\mathfrak{h}})$ as a formal Poisson Hopf algebra (the imaginary root vectors being obtained from them by means of the Poisson bracket) to get sure that the formulas above uniquely determine a continuous monomorphism $\mathcal{F} r_{\hat{\mathfrak{h}}}$, if any. Now consider $\mathfrak{F r}_{\hat{\mathfrak{g}}}: \mathfrak{U}_{\varepsilon}^{M^{\prime}}(\hat{\mathfrak{g}}) \longrightarrow \mathfrak{U}_{1}^{M^{\prime}}(\hat{\mathfrak{g}}) \cong U(\hat{\mathfrak{g}})$ (cf. (3.5)): its dual is a formal Hopf monomorphism $\mathfrak{U}_{1}^{M^{\prime}}(\hat{\mathfrak{g}})^{*} \longleftrightarrow \mathfrak{U}_{\varepsilon}^{M^{\prime}}(\hat{\mathfrak{g}})^{*}$; composing the latter with the isomorphisms $\mathcal{U}_{1}^{M}(\hat{\mathfrak{h}}) \stackrel{\cong}{\rightrightarrows} \mathfrak{U}_{1}^{M^{\prime}}(\hat{\mathfrak{g}})^{*}, \mathfrak{U}_{\varepsilon}^{M^{\prime}}(\hat{\mathfrak{g}})^{*} \stackrel{\cong}{\rightrightarrows} \mathcal{U}_{\varepsilon}^{M}(\hat{\mathfrak{h}}) \quad$ (given by specialized quantum Poisson pairings) provides a monomorphism $\mathcal{F} r_{\hat{\mathfrak{h}}}: \mathcal{U}_{1}^{M}(\hat{\mathfrak{h}}) \longleftrightarrow \mathcal{U}_{\varepsilon}^{M}(\hat{\mathfrak{h}})$, which by construction is continuous; moreover,

$$
\left\langle\mathcal{F} r_{\hat{\mathfrak{h}}}(h), g\right\rangle=\pi_{\varepsilon, \widehat{G}_{M}}\left(\mathcal{F} r_{\hat{\mathfrak{h}}}(h), g\right)=\pi_{1, \widehat{G}_{M}}\left(h, \mathfrak{F}_{\mathfrak{\mathfrak { g }}}(g)\right) \quad \forall h \in \mathcal{U}_{1}^{M}(\hat{\mathfrak{h}}), x \in \mathfrak{U}_{\varepsilon}^{M^{\prime}}(\hat{\mathfrak{g}})
$$

hence $\mathcal{F} r_{\hat{\mathfrak{h}}}$ is described by formulas above, as one sees at once just proceeding as for Theorem 6.7, by comparing PBW monomials - or simply root vectors - on one hand and their duals on the other: this also shows that (6.4) holds. So claim (a) is proved. 
Claim (b) follows from the analogous result for $\mathcal{U}_{\varepsilon}^{M}(\hat{\mathfrak{g}})$ (cf. [BK], $\left.\S \S 2-3\right)$ and comparison between $\mathcal{U}_{\varepsilon}^{M}(\hat{\mathfrak{g}})$ and $\mathcal{U}_{\varepsilon}^{M}(\hat{\mathfrak{h}})$. Claim (c) follows from (6.3) and (6.4). Finally, the span of $\left\{B_{\tau} \mid \tau \in \ell \mathbb{N}^{I_{\infty}}\right\}$ inside $\mathcal{U}_{\varepsilon}^{M}(\hat{\mathfrak{h}})$ equals the span of $\left\{L_{\mu} \mid \mu \in \ell \mathbb{N}^{I \infty} \cong \ell M_{+}\right\}$; this and the explicit description of the pseudobasis of $\mathcal{U}_{\varepsilon}^{M}(\hat{\mathfrak{h}})$ give claim $(d)$.

We call also $\mathfrak{F r}_{\hat{\mathfrak{h}}}$ and $\mathcal{F} r_{\hat{\mathfrak{h}}}$ quantum Frobenius morphisms.

6.10 Specializations of quantum Poisson pairings. From $\S \S 6.3-5$ we get that the Hopf pairings $\pi_{q, \widehat{H}_{M}}: \mathfrak{U}^{M^{\prime}}(\hat{\mathfrak{h}}) \otimes \mathcal{U}^{M}(\hat{\mathfrak{g}}) \longrightarrow R, \pi_{q, \widehat{G}_{M}}: \mathcal{U}^{M}(\hat{\mathfrak{h}}) \otimes \mathfrak{U}^{M^{\prime}}(\hat{\mathfrak{g}}) \longrightarrow R$ (cf. $\S 5.11)$ respectively specialize to the Hopf pairings $\pi_{\widehat{H}_{M}}: U(\hat{\mathfrak{h}}) \otimes F\left[\widehat{H}_{M}\right] \longrightarrow \mathbb{C}$, $\pi_{\widehat{G}_{M}}: F^{\infty}\left[\widehat{G}_{M}\right] \otimes U(\hat{\mathfrak{g}}) \longrightarrow \mathbb{C} ;$ in other words, $\left.\pi_{q, \widehat{H}_{M}}(\hat{h}, \tilde{g})\right|_{q=1}=\pi_{\widehat{H}_{M}}\left(\left.\hat{h}\right|_{q=1},\left.\tilde{g}\right|_{q=1}\right)$, $\left.\pi_{q, \widehat{G}_{M}}(\tilde{h}, \hat{g})\right|_{q=1}=\pi_{\widehat{G}_{M}}\left(\left.\tilde{h}\right|_{q=1},\left.\hat{g}\right|_{q=1}\right)$. Thus the quantum Poisson pairing is a quantization of the classical Hopf pairing on both our Poisson groups - maybe formal dual of each other. In addition we show that it can also be thought of as a quantization of the classical Poisson pairing $\pi_{\mathcal{P}}: \mathfrak{h} \otimes \mathfrak{g} \rightarrow \mathbb{C}$, and of new pairings between function algebras. We use notations $[]:,=m-m^{o p}, \nabla:=\Delta-\Delta^{o p}$ (superscript "op" denoting opposite operation).

First of all, we define a suitable grading on $\mathfrak{U}^{Q}(\hat{\mathfrak{g}})$ (as an $R$-module) by $\operatorname{deg}\left(\prod_{\alpha \in \widetilde{\Phi}_{+}} E_{\alpha}^{\left(e_{\alpha}\right)} \cdot \prod_{j \in I_{\infty}}\left(\begin{array}{c}K_{j} ; 0 \\ t_{j}\end{array}\right) K_{j}^{-E n t\left(t_{j} / 2\right)} \cdot \prod_{\alpha \in \widetilde{\Phi}_{+}} F_{\alpha}^{\left(f_{\alpha}\right)}\right):=\sum_{\alpha \in \widetilde{\Phi}_{+}}\left(e_{\alpha}+f_{\alpha}\right)+\sum_{j \in I_{\infty}} t_{j}$ and linear extension. Now let $R=: \mathfrak{U}_{0} \subset \mathfrak{U}_{1} \subset \cdots \subset \mathfrak{U}_{h} \subset \cdots\left(\subset \mathfrak{U}^{Q}(\hat{\mathfrak{g}})\right)$ be the associated filtration, and set $\partial(x):=h$ for all $x \in \mathfrak{U}_{h} \backslash \mathfrak{U}_{h-1}$; notice that a similar notion of degree exists for $U(\hat{\mathfrak{g}})$, defined by means of the filtration $U_{0} \subset U_{1} \subset \cdots \subset$ $U_{N} \subset \cdots \subset U(\hat{\mathfrak{g}})$ induced by the canonical filtration of $T(\mathfrak{g})$ (the tensor algebra on $\mathfrak{g})$, and similarly for $U(\hat{\mathfrak{g}}) \otimes U(\hat{\mathfrak{g}})$. Then $(q-1)^{\partial(g)} g \in \mathcal{U}^{Q}(\hat{\mathfrak{g}})$ for all $g \in \mathfrak{U}^{Q}(\hat{\mathfrak{g}})$, thus

$$
\pi_{q, \mathcal{P}}(h, g):=(q-1)^{+\partial(g)} \cdot \pi_{q}(h, g) \quad \forall h \in \mathfrak{U}^{Q}(\hat{\mathfrak{h}}), g \in \mathfrak{U}^{Q}(\hat{\mathfrak{g}})
$$

defines a perfect pairing $\pi_{q, \mathcal{P}}: \mathfrak{U}^{Q}(\hat{\mathfrak{h}}) \times \mathfrak{U}^{Q}(\hat{\mathfrak{g}}) \longrightarrow R$, which can be specialized at $q=1$.

Theorem 6.11. $\pi_{q, \mathcal{P}}: \mathfrak{U}^{Q}(\hat{\mathfrak{h}}) \times \mathfrak{U}^{Q}(\hat{\mathfrak{g}}) \longrightarrow R$ specializes at $q=1$ to a pairing

$$
\pi_{\mathcal{P}}: U(\hat{\mathfrak{h}}) \times U(\hat{\mathfrak{g}}) \longrightarrow \mathbb{C}
$$

which extends the Lie bialgebra pairing $\langle\rangle:, \mathfrak{h} \otimes \mathfrak{g} \longrightarrow \mathbb{C}$ (cf. 1.2) and is such that

$$
\begin{aligned}
& \pi_{\mathcal{P}}(\alpha \cdot x+\beta \cdot y, z)=\alpha \cdot \pi_{\mathcal{P}}(x, z)+\beta \cdot \pi_{\mathcal{P}}(y, z) \\
& \pi_{\mathcal{P}}(x, \alpha \cdot u+\beta \cdot v)=\alpha \cdot \pi_{\mathcal{P}}(x, u)+\beta \cdot \pi_{\mathcal{P}}(x, v) \\
& \pi_{\mathcal{P}}(x \cdot y, z)=\pi_{\mathcal{P}}(x \otimes y, \Delta(z)), \quad \pi_{\mathcal{P}}(x, z \cdot w)=\pi_{\mathcal{P}}(\Delta(x), z \otimes w) \\
& \pi_{\mathcal{P}}([x, y], z)=\pi_{\mathcal{P}}(x \otimes y, \delta(z)), \quad \pi_{\mathcal{P}}(x,[z, w])=\pi_{\mathcal{P}}(\delta(x), z \otimes w)
\end{aligned}
$$

for all $\alpha, \beta \in \mathbb{C}, x, y \in U(\hat{\mathfrak{h}}), z, w, u, v \in U(\hat{\mathfrak{g}})$ such that $\partial(\alpha \cdot u+\beta \cdot v)=\partial(u)=\partial(v)$. 
Proof. Let $x \in U(\hat{\mathfrak{h}}), z \in U(\hat{\mathfrak{g}})$, and pick $x^{\prime} \in \mathfrak{U}^{Q}(\hat{\mathfrak{h}}), z^{\prime} \in \mathfrak{U}^{Q}(\hat{\mathfrak{g}})$, such that $x=\left.x^{\prime}\right|_{q=1}$, $z=\left.z^{\prime}\right|_{q=1}$. By definition, $\pi_{\mathcal{P}}(x, z)$ is given by

$$
\pi_{\mathcal{P}}(x, z):=\left.\pi_{q, \mathcal{P}}\left(x^{\prime}, z^{\prime}\right)\right|_{q=1}=\left.\left((q-1)^{\partial\left(z^{\prime}\right)} \cdot \pi_{q}\left(x^{\prime}, z^{\prime}\right)\right)\right|_{q=1}
$$

in particular, we can select $x^{\prime}$ and $z^{\prime}$ such that $\partial\left(x^{\prime}\right)=\partial(x), \partial\left(z^{\prime}\right)=\partial(z)$. Now, the first two lines in (6.5) follow directly from similar properties for $\pi_{q, \mathcal{P}}$, which are implied by definitions. Second, definitions and Leibnitz' and co-Leibnitz' rules imply

$$
\begin{gathered}
\partial(x \cdot y)=\partial(x)+\partial(y)=\partial(x \otimes y), \quad \partial(\Delta(x))=\partial(x) \\
\partial(\delta(x))=\partial(x)+1, \quad \partial([x, y])=\partial(x)+\partial(y)-1
\end{gathered}
$$

for all $x, y \in U(\hat{\mathfrak{g}})$ provided that $[x, y] \neq 0$ (to be complete we may set $\partial(0):=-\infty$ ); using these identities and Leibnitz' and co-Leibnitz' rules we easily reduce to prove that the remaining identities in (6.5) do hold in degree 1, i.e. for $x, y \in \hat{\mathfrak{h}}$ and $z, w \in \hat{\mathfrak{g}}$ : but this again follows from definition. Finally to prove that $\pi_{\mathcal{P}}$ is an extension of the classical Poisson pairing it is enough to perform a computation on Chevalley generators, which is completely straightforward.

6.12 The pairing $F^{\infty}\left[\widehat{G}_{M^{\prime}}\right] \times F\left[\widehat{H}_{M}\right] \longrightarrow \mathbb{C}$. The construction in $\S 6.10$ can be reversed as follows. Define a grading on $\mathcal{U}^{M}(\hat{\mathfrak{g}})$ (as a $R$-module) by

$$
\operatorname{deg}\left(\prod_{\alpha \in \widetilde{\Phi}_{+}} \bar{E}_{\alpha}^{e_{\alpha}} \cdot \prod_{j \in I_{\infty}}\left(M_{j}^{ \pm 1}-1\right)^{m_{j}} \cdot \prod_{\alpha \in \widetilde{\Phi}_{+}} \bar{F}_{\alpha}^{f_{\alpha}}\right):=\sum_{\alpha \in \widetilde{\Phi}_{+}}\left(e_{\alpha}+f_{\alpha}\right)+\sum_{j \in I_{\infty}} m_{j}
$$

and linear extension; then let $R=: \mathcal{U}_{0} \subset \mathcal{U}_{1} \subset \cdots \subset \mathcal{U}_{h} \subset \cdots\left(\subset \mathcal{U}^{M}(\hat{\mathfrak{g}})\right)$ be the associated filtration, and set $\partial(x):=h$ for all $x \in \mathcal{U}_{h} \backslash \mathcal{U}_{h-1}(h \in \mathbb{N})$. Finally define

$$
\pi_{q}^{\mathcal{P}}(h, g):=(q-1)^{-\partial(g)} \cdot \pi_{q}(h, g) \quad \forall h \in \mathcal{U}^{M^{\prime}}(\hat{\mathfrak{h}}), g \in \mathcal{U}^{M}(\hat{\mathfrak{g}}) ;
$$

this yields a perfect pairing $\pi_{q}^{\mathcal{P}}: \mathcal{U}^{M^{\prime}}(\hat{\mathfrak{h}}) \times \mathcal{U}^{M}(\hat{\mathfrak{g}}) \longrightarrow R$, to be specialized at $q=1$.

Teorema 6.13. $\pi_{q}^{\mathcal{P}}: \mathcal{U}^{M^{\prime}}(\hat{\mathfrak{h}}) \times \mathcal{U}^{M}(\hat{\mathfrak{g}}) \longrightarrow R$ specializes at $q=1$ to a pairing

$$
\pi^{\mathcal{P}}: F^{\infty}\left[\widehat{G}_{M^{\prime}}\right] \times F\left[\widehat{H}_{M}\right] \longrightarrow \mathbb{C}
$$

such that

$$
\begin{gathered}
\pi_{\tau}^{\mathcal{P}}(\alpha \cdot x+\beta \cdot y, z)=\alpha \cdot \pi_{\tau}^{\mathcal{P}}(x, z)+\beta \cdot \pi_{\tau}^{\mathcal{P}}(y, z) \\
\pi_{\tau}^{\mathcal{P}}(x, \alpha \cdot u+\beta \cdot v)=\alpha \cdot \pi_{\tau}^{\mathcal{P}}(x, u)+\beta \cdot \pi_{\tau}^{\mathcal{P}}(x, v) \\
\pi_{\tau}^{\mathcal{P}}(x \cdot y, z)=\pi_{\tau}^{\mathcal{P}}(x \otimes y, \Delta(z)), \quad \pi_{\tau}^{\mathcal{P}}(x, z \cdot w)=\pi_{\tau}^{\mathcal{P}}(\Delta(x), z \otimes w) \\
\pi_{\tau}^{\mathcal{P}}(\{x, y\}, z)=\pi_{\tau}^{\mathcal{P}}(x \otimes y, \nabla(z)), \quad \pi_{\tau}^{\mathcal{P}}(x,\{z, w\})=\pi_{\tau}^{\mathcal{P}}(\nabla(x), z \otimes w)
\end{gathered}
$$

for all $\alpha, \beta \in \mathbb{C}, x, y \in F^{\infty}\left[\widehat{G}_{M^{\prime}}\right], z, u, v \in F\left[\widehat{H}_{M}\right]$ such that $\partial(\alpha \cdot u+\beta \cdot v)=\partial(u)=$ $\partial(v)$ (with $\partial(x):=\partial\left(x^{\prime}\right)$ for any $x^{\prime} \in \mathcal{U}^{M}(\hat{\mathfrak{g}})$ such that $\left.\left.x^{\prime}\right|_{q=1}=x\right)$.

Proof. Just mimick the proof of Theorem 6.11 above. 
$\underline{\text { Remark: }}$ If we extend $R$ by adding a $\Delta_{\infty}$-th root of unity $\left(\Delta_{\infty}\right.$ as in $\left.\S 1.1\right)$ we can perform the previous construction for any pair of lattices $M_{1}, M_{2}$ such that $Q_{\infty} \leq$ $M_{1}, M_{2} \leq P_{\infty}$, thus getting corresponding pairings like the one of Theorem 6.13.

\title{
REFERENCES
}

[Be1] J. Beck, Braid group action and quantum affine algebras, Comm. Math. Phys. 165 (1994), 555-568.

[Be2] J. Beck, Convex bases of PBW type for quantum affine algebras, Comm. Math. Phys. 165 (1994), $193-199$.

[BK] J. Beck, V. G. Kac, Finite dimensional representations of quantum affine algebras at roots of 1 , J. Amer. Math. Soc. 9 (1996), 391-423.

[Bo] N. Bourbaki, Groupes et algèbres de Lie, Chapitres 4-6, Hermann, Paris, 1968.

[CP] V. Chari, A. Pressley, A guide to Quantum Groups, Cambridge University Press, Cambridge, 1994.

[Da] I. Damiani, La $R$-matrice pour les algèbres quantiques de type affine non tordu, Ann. Scient. Éc. Norm. Sup., $4^{e}$ série, 31 (1998), 493-523.

[Di] J. Dieudonné, Introduction to the theory of formal groups, Pure Appl. Math. 20, Marcel Dekker, Inc. New York, 1973.

[DL] C. De Concini, V. Lyubashenko, Quantum function algebra at roots of 1, Adv. Math. 108 (1994), 205-262.

[Dr] V. G. Drinfel'd, Quantum groups, Proc. ICM Berkeley 1 (1986), 789-820.

[Ga1] F. Gavarini, Quantization of Poisson groups, Pac. J. Math. 186 (1998), 217-266.

[Ga2] F. Gavarini, A PBW basis for Lusztig's form of untwisted affine quantum groups, Commun. Algebra 27 (1999), no. 2, 903-918.

[Ga3] F. Gavarini, The quantum duality principle, preprint math.QA/9909071.

[Lu1] G. Lusztig, Quantum groups at roots of 1, Geom. Dedicata 35 (1990), 89-113.

[Lu2] G. Lusztig, Introduction to quantum groups, Progr. Math. 110, Birkhäuser, Boston, 1993.

[Ta] T. Tanisaki, Killing forms, Harish-Chandra Isomorphisms, and Universal R-Matrices for Quantum Algebras, Internat. J. Modern Phys. A 7, Suppl. 1B (1992), 941-961.

\author{
Università Degli Studi di Roma "Tor Vergata" \\ DipARTIMENTO Di MATEMATICA \\ Via Della Ricerca Scientifica 1 \\ I-00133 ROMA — ITALY \\ E-MAIL: GAVARINI@MAT.UNIROMA2.IT
}

